\title{
Imersões Justas de Variedades em Espaços Euclideanos
}

\author{
antonio Carlos Nogueira
}

\author{
Orientação: \\ Profa. Dra. Maria Aparecida Soares Ruas
}

Dissertaçâo apresentada ao Instituto de Ciências Matemáticas de São Carlos - USP, como parte dos requisitos para obtenção do título de "Mestre em Ciências - Área: Matemática".

USP - São Carlos

Maio de 1993 
À minha família 


\section{Agradecimentos ${ }^{1}$}

À Cidinha pelo incentivo, pelos conselhos e pela sua dedicação durante todo o desenvolver deste trabalho.

A todos os professores com os quais tive o prazer de trabalhar.

A meus colegas de mestrado e de sala, pelo companheirismo.

Aos vigias Sr. Nelson, Sr. Alcides e Sr. Antonio Carlos (Toninho) por estarem sempre à disposição.

Agradeço de modo especial à Angélica pelo seu apoio e principalmente pelo seu amor.

À minha família pelo carinho, amor e compreensão.

Finalmente, agradeço a Deus, pois sem Ele, nada seria possível.

\footnotetext{
${ }^{1}$ Este trabalho teve suporte financeiro do $\mathrm{CNPq}$
} 


\begin{abstract}
A mapping $f: M \rightarrow \mathbf{E}^{m}$, from a topological compact, connected space into Euclidean space is tight if for every closed semi-space $h \subset \mathbf{E}^{m}$, the inclusion $f^{-1}(h) \hookrightarrow M$ induces a monomorphism in Cech $\mathbf{Z}_{2}$-homology. In this work we consider mappings with this property, emphasizing the study of properties of tight immersions of manifolds into Euclidean space. For 2-manifolds tightness is equivalent to the total absolute curvature being minimal.

Our main purpose is to discuss the existence of tight immersions for surfaces into $\mathbf{E}^{3}$. It follows from the work of $\mathrm{N}$. Kuiper, and a recent result of $\mathrm{F}$. Haab that all surfaces admit a tight immersion, except the projective plane $(\chi=1)$, the Klein's bottle $(\chi=0)$ and the projective plane with one handle $(\chi=-1)$. We also study a special generic family of $C^{\infty}$-tight mappings from the projective plane into $\mathbf{E}^{3}$.
\end{abstract}




\section{Resumo}

Uma aplicação $f: M \rightarrow \mathbf{E}^{m}$, de um espaço topológico compacto e conexo em um espaço Euclideano é justa se para todo semi-espaço fechado $h \subset \mathbf{E}^{m}$, a inclusão $f^{-1}(h) \hookrightarrow M$ induz um monomorfismo em $\mathbf{Z}_{2}$-homologia de Čech. Neste trabalho consideramos aplicações com esta propriedade, enfatizando o estudo de propriedades de imersões justas de variedades em espaços euclideanos. Para variedades de dimensão 2 justeza é equivalente a curvatura total absoluta sendo mínima.

Nosso principal objetivo é discutir a existência de imersões justas para superfícies em $\mathbf{E}^{3}$. Segue do trabalho de $\mathrm{N}$. Kuiper, e de um resultado recente de F. Haab, que todas as superfícies, exceto o plano projetivo $(\chi=1)$, a garrafa de Klein $(\chi=0)$ e o plano projetivo com uma alça $(\chi=-1)$, admitem imersão justa em $\mathbf{E}^{3}$. Estudamos também uma família genérica especial de aplic cões justas $C^{\infty}$-estáveis do plano projetivo em $\mathbf{E}^{3}$. 


\section{Conteúdo}

Introdução 1

1 Preliminares 4

1.1 Teoria de Pontos Críticos . . . . . . . . . . . . . . 4

1.2 Conexões Afins . . . . . . . . . . . . . . . . 9

1.3 A Segunda Forma Fundamental . . . . . . . . . . . . . . . 10

1.4 A aplicação de Gauss . . . . . . . . . . . . . . . . . . . . . 13

2 Aplicações Justas $\quad 18$

2.1 Curvatura Total Absoluta . . . . . . . . . . . . 18

2.2 Hipersuperfícies Convexas ................ . 25

2.3 Aplicações Justas e Imersões . . . . . . . . . . . . . . . . . . . 26

3 A Propriedade dos Dois Pedaços

4 Top-Sets e Teorema de Chern-Lashof 45

4.1 Top-sets ......................... 45

4.2 O Teorema de Chern-Lashof . . . . . . . . . . . 55

$\begin{array}{llr}5 & \text { Superfícies Justas em } \mathbf{E}^{3} & \mathbf{5 8}\end{array}$ 
5.1 Superfícies Justas . . . . . . . . . . . . . . . 58

5.2 Top-Ciclos . . . . . . . . . . . . . . . . . . 64 64

5.3 Imersões Justas de Superfícies com Característica de Euler impar $\mathrm{em} \mathbf{E}^{3} \ldots \ldots \ldots \ldots \ldots \ldots \ldots$

5.4 Aplicações $C^{\infty}$-Estáveis Justas . . . . . . . . . . . . . 72

$\begin{array}{lr}\text { Bibliografia } & \mathbf{8 1}\end{array}$ 


\section{Introdução}

Seja $\gamma:[0,1] \rightarrow \mathbf{E}^{3}$ uma curva fechada simples parametrizada pelo comprimento de arco. Denotemos por $k(s)$ a curvatura de $\gamma(s)$. A curvatura total absoluta de $\gamma$ é definida pela integral

$$
\tau(\gamma)=\int_{0}^{1}|k(s)| d s .
$$

Um resultado clássico da Geometria Diferencial devido a Fenchel é o seguinte teorema, cuja demonstração pode ser encontrada em [4],

Teorema 0.1 (Fenchel) A curvatura total absoluta de uma curva $\gamma$ fechada e simples em $\mathbf{E}^{3} e ́ \geq 2$ e, a igualdade se verifica se, e somente se, $\gamma$ é convexa.

Suponhamos agora que $M^{2}$ é uma superfície orientável e $f: M^{2} \rightarrow \mathbf{E}^{3}$ é uma imersão. Seja $K$ a curvatura Gaussiana de $f$. Como no caso de curvas podemos considerar a curvatura total absoluta de $f$ dada por

$$
\tau(M, f)=\int_{M}|K| d A,
$$

onde $d A$ é o elemento de volume de $M$. Com o auxílio da teoria de pontos críticos de Morse é possivel mostrar que $\tau(M, f) \geq 2 \pi(4-\chi(M))$, onde $\chi(M)$ é a característica de Euler de $M$. Surge então, naturalmente, a seguinte questão:

Dada uma superfície $M^{2}$, existe uma imersão $f: M^{2} \rightarrow \mathbf{E}^{3}$ tal que $\tau(M, f)$ atinge o valor mínimo possivel, isto é, tal que $\tau(M, f)=2 \pi(4-\chi(M))$ ?

Nosso objetivo neste trabalho será responder a esta questão, concluindo a existência ou não de tais imersôes para todas as variedades de dimensão 2 , tanto orientáveis como não orientáveis. Para isto, tomamos como referência o livro Tight and taut immersions of manifolds de Cecil e Ryan [6]. Se existir uma tal 
imersão $f: M^{2} \rightarrow \mathbf{E}^{3}$ diremos que $f$ é uma imersão justa. O problema acima pode ser formulado de uma maneira mais geral para imersões $f: M^{n} \rightarrow \mathbf{E}^{m}$ com $m, n$ arbitrários.

Para atacar esta questão começamos, no capítulo 1, apresentando alguns resultados centrais da teoria de pontos críticos, como o lema de Morse e as desigualdades de Morse. Em seguida definimos a $2^{a}$ forma fundamental de uma imersão $f: M^{n} \rightarrow \mathbf{E}^{m}$ e a aplicaçãa normal de Gauss associada a $f$. Então, finalizando o capitulo relacionamos os valores regulares da aplicação de Gauss com as singularidades não degeneradas de funções altura.

No capítulo 2 definimos a curvatura de Lipschitz-Killing, que denotaremos por $G$, a qual generaliza a curvatura Gaussiana de superfícies ([22]). Definimos então a curvatura total absoluta de $f$ pela integral

$$
\tau(M, f)=\int_{B}|G| d A,
$$

onde $B$ é o fibrado normal unitário de $f$ e $d A$ seu elemento de volume. Uma expressão simples em termos de pontos críticos de funções altura é obtida para $\tau(M, f)$ e com isto é possivel mostrar que

$$
\inf _{f} \tau(M, f)=\gamma(M)
$$

onde $\gamma(M)$ é o número de Morse de $M$. Em seguida apresentamos a definição de justeza de Kuiper [13] dada em termos de injetividade em homologia e mostramos que para variedades que satisfazem a condição $\beta\left(M ; \mathbf{Z}_{2}\right)=\gamma(M)$ justeza é equivalente a imersão com curvatura total absoluta minima, onde $\beta\left(M ; \mathbf{Z}_{2}\right)$ é a soma dos números de Betti de $M$ com coeficientes em $\mathbf{Z}_{2}$.

No capitulo 3 introduzimos a propriedade dos dois pedaços (PDP) de Banchoff, que em dimensão 2 coincide com justeza. Muitas vezes será conveniente utilizar esta noção devido a seu significado geométrico bastante simples. Relacionamos aqui os principais resultados sobre imersões PDP, dentre os quais destacamos o teorema da codimensão de Kuiper, que estabelece um limite superior para a codimensão de imersões PDP substanciais.

No capítulo 4 introduzimos a noção de top-sets a qual será utilizada para demonstrar o conhecido teorema de Chern-Lashof. Este teorema generaliza o teorema de Fenchel e estabelece que uma imersão $f: S^{n} \rightarrow \mathbf{E}^{m}$ é tal que $\tau(M, f)=2$ se, e somente se, $m=n+1$ e $f$ mergulha $S^{n}$ como uma hipersuperfície convexa em $\mathbf{E}^{n+1}$.

Finalmente, no capitulo 5 damos a classificação de superfícies justas. Começamos construindo imersões (e mergulhos) para todas as superfícies orientáveis em 
$\mathbf{E}^{3}$. Apresentamos também os resultados sobre existência de imersões justas para todas as superfícies não orientávejs com característica de Euler $\chi \leq-3$. A não existência de imersões justas, mesmo topológicas, para o plano projetivo $(\chi=1)$ e para a garrafa de Klein $(\chi=0)$ também são demonstradas. O problema de completar a lista de variedades que admitem imersões justas foi recentemente resolvido por $\mathrm{F}$. Haab [11], que mostrou que o plano projetivo com uma alça $(\chi=-1)$ não admite imersão justa em $\mathbf{E}^{3}$. Finalizando este capítulo estudamos uma família genérica especial de aplicações $C^{\infty}$-justas do plano projetivo $\mathbf{P}^{2} \mathrm{em}$ $\mathbf{E}^{3}$. Fazemos uma análise detalhada desta família e verificamos que a mesma apresenta singularidades $C^{\infty}$-estáveis, exceto para um valor $t_{0}$ do parâmetro. Para $t<t_{0}$ as imagens $g_{t}\left(\mathbf{P}_{2}\right)$ são as superfícies singulares denominadas CrossCaps de Steiner que apresentam 2 cross-caps, enquanto que para $t>t_{0}$ as imagens $g\left(\mathbf{P}^{2}\right)$ são as superfícies singulares denominadas Superfícies Romanas de Steiner e apresentam 6 cross-caps. 


\section{Capítulo 1}

\section{Preliminares}

Neste capítulo introdutório apresentaremos alguns conceitos básicos da Geometria Riemanniana tais como conexões Riemannianas, segunda forma fundamental de uma imersão e a aplicação de Gauss. O objetivo central deste capítulo é relacionar os valores regulares da aplicação de Gauss com as singularidades não degeneradas das funções altura. Para isto, faremos uso da teoria de pontos críticos.

\subsection{Teoria de Pontos Críticos}

Nesta seção relacionaremos alguns dos principais resultados da teoria de pontos críticos, destacando o conhecido Lema de Morse e as desigualdades de Morse.

Seja $\phi: M \rightarrow \mathbf{R}$ uma função diferenciável. Um ponto $x \in M$ é dito um ponto crítico de $\phi$ se a diferencial de $\phi$ em $x, \phi_{*}: T_{x} M \rightarrow T_{\phi(x)} \mathbf{R}$, é a aplicação nula. Em um sistema de coordenadas $\left(u_{1}, \ldots, u_{n}\right)$ em torno de $x$, isto significa que

$$
\frac{\partial \phi(x)}{\partial u_{1}}=\ldots=\frac{\partial \phi(x)}{\partial u_{n}}=0 .
$$

O número real $f(x)$ é dito um valor crítico de $\phi$. Dizemos que um ponto crítico $x \in M$ é não-degenerado se a matriz

$$
\left(\frac{\partial^{2} \phi}{\partial u_{i} \partial u_{j}}(x)\right)
$$


é não-singular. Esta matriz é denominada a Hessiana de $\phi$ em $x$ e será denotada por $H_{x}$.

Definição 1.1 Uma função $\phi: M \rightarrow \mathbf{R}$ que tem somente pontos críticos nãodegenerados é dita uma função de Morse ou uma função não-degenerada. $O$ índice de $\phi$ em um ponto crítico não-degenerado $x \in M$ é o número de autovalores negativos da Hessiana $H_{x}$.

Um importante resultado na teoria de pontos críticos é o Lema de Morse que mostra que o comportamento de $\phi$ em uma vizinhança de um ponto crítico não-degenerado é completamente determinado pelo seu índice.

Lema 1.1 (Morse) Seja $x \in M$ um ponto crítico da função de Morse $\phi: M \rightarrow \mathbf{R}$ de indice $\lambda$. Então existe um sistema de coordenadas locais $\left(u_{1}, \ldots, u_{n}\right)$ em uma vizinhança $U$ de $x$ com $u_{i}(x)=0$, tal que a seguinte identidade

$$
\phi=\phi(p)-\left(u_{1}\right)^{2}-\cdots-\left(u_{\lambda}\right)^{2}+\left(u_{\lambda+1}\right)^{2}+\cdots+\left(u_{n}\right)^{2}
$$

se verifica em $U$.

Dada $\phi: M \rightarrow \mathbf{R}$ e $a \in \mathbf{R}$ considere o conjunto

$$
M_{a}=\{x \in M ; \phi(x) \leq a\} .
$$

Os seguintes resultados sobre a topologia de $M$ podem ser encontrados em Milnor [21].

Teorema 1.1 Se $a<b$ e $\phi^{-1}[a, b]$ é compacto e não contém pontos críticos de $\phi$ então $M_{a}$ é difeomorfo a $M_{b}$. Além disso, $M_{a}$ é retrato por deformação de $M_{b}$, isto é, a inclusẫo $M_{a} \hookrightarrow M_{b}$ é equivalência de homotopia.

Teorema 1.2 Sejam $\phi: M \rightarrow \mathbf{R}$ uma função diferenciável e $p \in M$ um ponto crítico não-degenerado de $\phi$ com indice $\lambda$. Seja $\phi(p)=c$ e suponha que $\phi^{-1}[c-\epsilon, c+\epsilon]$ é compacto e não contenha pontos críticos de $\phi$ além de $p$, para algum $\epsilon>0$. Então para $\epsilon$ suficientemente pequeno, $M_{c+\epsilon}$ tem o mesmo tipo de homotopia de $M_{c-c}$ com uma $\lambda$-célula adicionada. 
Teorema 1.3 Se $\phi: M \rightarrow \mathbf{R}$ é diferenciável com pontos críticos não-degenerados e, se cada $M_{a}$ é compacto, entâo $M$ tem o tipo de homotopia de um $C W$-complexo, com uma célula de dimensão $\lambda$ para cada ponto crítico de índice $\lambda$.

Corolário 1.1 $M_{a}$ tem o tipo de homotopia de um CW-complexo, com uma célula de dimensão $\lambda$ para cada ponto crítico de índice $\lambda$ em $M_{a}$.

Como consequência dos teoremas acima temos o seguinte

Teorema 1.4 (Reeb) Se $M^{n}$ é uma variedade compacta e $f: M \rightarrow \mathbf{R}$ é uma função diferenciável em $M$ com somente dois pontos críticos, ambos não degenerados, então $M$ é homeomorfa a uma n-esfera.

Descreveremos a seguir relações entre a topologia de uma variedade $M$ e os pontos críticos de uma função de Morse definida em $M$, relações estas que serão expressas em termos de desigualdades, a saber, as conhecidas desigualdades de Morse.

Seja $M$ uma variedade compacta e $\phi: M \rightarrow \mathbf{R}$ uma função de Morse. Denotemos por $\mu_{\lambda}$ o número de pontos críticos de indice $\lambda$ de $\phi$ e seja $\beta_{i}(M, F)$ o i-ésimo número de Betti de $M$ com relação ao corpo $F$. Sejam $a_{0}<a_{1}<\ldots a_{s}$ números reais tais que $M_{a_{i}}$ contém exatamente i pontos críticos e, $M_{a_{s}}=M$, $M_{a_{0}}=\emptyset$. Note que $M_{a_{i-1}} \subset M_{a_{i}}$ e, $M_{a_{i}}-M_{a_{i-1}}$ tem um único ponto crítico de índice $\lambda_{i}$ e, além disso, $M_{a i}$ é obtido de $M_{a_{i-1}}$ pela adjunção de uma $\lambda_{i}$-célula (teorema 1.3). Segue que

$$
\operatorname{rank}\left(H_{k}\left(M_{a_{i}}, M_{a i-1}\right)\right)= \begin{cases}0, & \text { se } k \neq \lambda_{i} \\ 1, & \text { se } k=\lambda_{i}\end{cases}
$$

Consideremos a sequência exata do par $\left(M_{a_{i}}, M_{a_{i-1}}\right)$ dada abaixo

$$
\ldots \stackrel{\partial_{k+1}}{\rightarrow} H_{k}\left(M_{a_{i-1}}\right) \stackrel{j_{k}^{i}}{\rightarrow} H_{k}\left(M_{a_{i}}\right) \stackrel{l_{k}}{\rightarrow} H_{k}\left(M_{a_{i}}, M_{a_{i-1}}\right) \stackrel{\partial_{k}}{\rightarrow} H_{k-1}\left(M_{a_{i-1}}\right) \rightarrow \ldots
$$

e vamos introduzir as seguintes notações:

$$
\begin{aligned}
\beta_{k}^{i} & =\operatorname{rank}\left(H_{k}\left(M_{a_{i}}\right)\right) \\
C_{k}^{i} & =\operatorname{rank}\left(H_{k}\left(M_{a_{i}}, M_{a_{i-1}}\right)\right)
\end{aligned}
$$




$$
\begin{aligned}
b_{k}^{i} & =\operatorname{rank}\left(\partial_{k+1}\right) \\
\beta_{k} & =\operatorname{rank}\left(H_{k}(M)\right)=\beta_{k}^{s} \\
C_{k} & =\sum_{i=1}^{s} C_{k}^{i}=\mu_{k} \\
b_{k} & =\sum_{i=1}^{s} b_{k}^{i} .
\end{aligned}
$$

Da exatidão da sequência acima temos:

$$
\begin{aligned}
\beta_{k}^{i} & =\operatorname{rank}\left(H_{k}\left(M_{a_{i}}\right)\right)=\operatorname{rank}\left(l_{k}\right)+\operatorname{nul}\left(l_{k}\right) \\
& =\operatorname{ran}\left(l_{k}\right)+\operatorname{rank}\left(j_{k}^{i}\right)=\operatorname{nul}\left(\partial_{k}\right)+\operatorname{rank}\left(j_{k}^{i}\right) \\
& =\operatorname{rank}\left(H_{k}\left(M_{a_{i}}, M_{a_{i-1}}\right)\right)-\operatorname{rank}\left(\partial_{k}\right)+\operatorname{rank}\left(H_{k}\left(M_{a_{i-1}}\right)\right)-\operatorname{rank}\left(\partial_{k+1}\right) \\
& =C_{k}^{i}-b_{k-1}^{i}+\beta_{k}^{i-1}-b_{k}^{i} .
\end{aligned}
$$

Portanto,

$$
C_{k}^{i}=\beta_{k}^{i}-\beta_{k}^{i-1}+b_{k}^{i}+b_{k-1}^{i} .
$$

Somando em $i$, obtemos:

$$
C_{k}=\beta_{k}+b_{k}+b_{k-1},
$$

e daí, segue que $C_{k} \geq \beta_{k}$. Além disso,

$$
\begin{aligned}
C_{k}-C_{k-1}+\cdots+(-1)^{k} C_{0} & =\left(\beta_{k}+b_{k}+b_{k-1}\right)+\cdots+(-1)^{k}\left(\beta_{0}+b_{0}\right) \\
& =\beta_{k}-\beta_{k-1}+\cdots+(-1)^{k} \beta_{0}+b_{0},
\end{aligned}
$$

e, portanto

$$
C_{k}-C_{k-1}+\cdots+(-1)^{k} C_{0} \geq \beta_{k}-\beta_{k-1}+\cdots+(-1)^{k} \beta_{0} .
$$

Para $k=n+1$ temos $C_{k}=0$ e, portanto $\beta_{k}=0$. Assim, a desigualdade acima fica

$$
-C_{n}+C_{n-1}+\cdots+(-1)^{n+1} C_{0} \geq-\beta_{n}+\beta_{n-1}+\cdots+(-1)^{n+1} \beta_{0},
$$

e, por outro lado, para $k=n$, temos

$$
C_{n}-C_{n-1}+\cdots+(-1)^{n} C_{0} \geq \beta_{n}-\beta_{n-1}+\cdots+(-1)^{n} \beta_{0} .
$$


Logo, temos a igualdade

$$
C_{n}-C_{n-1}+\cdots+(-1)^{n} C_{0}=\beta_{n}-\beta_{n-1}+\cdots+(-1)^{n} \beta_{0}
$$

ou seja,

$$
\begin{aligned}
\mu_{0}-\mu_{1}+\cdots+(-1)^{n} \mu_{n} & =\beta_{0}-\beta_{1}+\cdots+(-1)^{n} \beta_{n} \\
& =\chi(M) .
\end{aligned}
$$

Demonstramos assim o seguinte

Teorema 1.5 (Desigualdades de Morse) Seja $\phi: M \rightarrow \mathbf{R}$ uma função de Morse definida na variedade compacta $M$. Então as seguintes desigualdades se verificam

$$
\begin{aligned}
\mu_{k} & \geq \beta_{k} \\
\mu_{k}-\mu_{k-1}+\cdots+(-1)^{k} \mu_{0} & \geq \beta_{k}-\beta_{k-1}+\cdots+(-1)^{k} \beta_{0}, \\
\mu_{0}-\mu_{1}+\cdots+(-1)^{n} \mu_{n} & =\beta_{0}-\beta_{1}+\cdots+(-1)^{n} \beta_{n} \\
& =\chi(M),
\end{aligned}
$$

onde $\mu_{k}$ denota o número de pontos críticos de índice $k$ de $\phi$ e $\beta_{k}$ é o k-ésimo número de Betti de $M$.

A força das desigualdades de Morse está no fato de que, fixado um grupo $G$, o lado direito depende somente da topologia de $M$, pois é uma combinação dos números de Betti de $M$ em relação a este grupo $G$, os quais são invariantes topológicos, enquanto que o lado esquerdo depende somente da função $\phi \mathrm{em}$ vizinhanças arbitrárias de seus pontos críticos.

Seja $\gamma(M)$ o número de Morse de $M$, isto é,

$$
\gamma(M)=\min \{\mu(\phi) ; \phi: M \rightarrow \mathbf{R} \text { é de Morse em } M\}
$$

onde $\mu(\phi)$ é o número de pontos críticos de $\phi$. Um corolário das desigualdades de Morse é o seguinte

\section{Corolário 1.2}

$$
\beta(M)=\sum_{k=0}^{n} \beta_{k}(M) \leq \gamma(M)
$$




\section{Demonstração:}

Seja $\phi: M \rightarrow \mathbf{R}$ uma função de Morse. Então, pelas desigualdades de Morse, temos $\mu_{k}(\phi) \geq \beta_{k}$. Portanto,

$$
\sum_{k=0}^{n} \beta_{k}(M) \geq \sum_{k=0}^{n} \mu_{k}(\phi)=\mu(\phi) .
$$

Como $\gamma(M)$ é o mínimo dos números $\mu(\phi)$ tal que $\phi$ é de Morse, segue que $\gamma(M) \geq \beta(M)$.

\subsection{Conexões Afins}

Seja $M$ uma variedade diferenciável e denotemos por $\mathcal{X}(M)$ o conjunto dos campos de vetores $C^{\infty}$ em $M$ e por $\mathcal{D}(M)$ o anel das funções $C^{\infty}$ definidas em $M$. Notemos que $\mathcal{X}(M)$ é um módulo sobre $\mathcal{D}(M)$.

Definição 1.2 Uma conexão afim em $M$ é uma aplicação

$$
\nabla: \mathcal{X}(M) \times \mathcal{X}(M) \rightarrow \mathcal{X}(M)
$$

indicada por

$$
(X, Y) \rightarrow \nabla_{X} Y
$$

que satisfaz as seguintes condições:

(1) $\nabla_{f X+g Y} Z=f \nabla_{X} Z+g \nabla_{Y} Z$;

(2) $\nabla_{X}(Y+Z)=\nabla_{X} Y+\nabla_{X} Z$;

(3) $\nabla_{X}(f Y)=f \nabla_{X} Y+X(f) Y$;

onde $f, g \in \mathcal{D}(M)$ e $X, Y, Z \in \mathcal{X}(M)$.

Note que se $X \in \mathcal{X}^{\prime}(M)$ e $f \in \mathcal{D}(M)$, então $f X$ é o campo vetorial definido por

$$
(f X)_{q}=f(q) X(q)
$$

e, para cada $p \in M, X_{p} f$ denota a derivada direcional de $f$ segundo a direção $p$.

Definição 1.3 Seja $M$ uma variedade diferenciável com uma conexão afim $\nabla e$ uma métrica Riemanniana que será denotada por $g$. A conexão é dita compatível com a métrica se para todo $X, Y, Z \in \mathcal{X}(M)$ tem-se

$$
X g(Y, Z)=g\left(\nabla_{X} Y, Z\right)+g\left(Y, \nabla_{X} Z\right)
$$


Definição 1.4 Uma conexão afim numa variedade diferenciável $M$ é dita simétrica se

$$
\nabla_{X} Y-\nabla_{Y} X=[X, Y]
$$

$\forall X, Y \in \mathcal{X}(M)$, onde [,] denota o colchete de Lie.

Um dos resultados centrais da Geometria Riemanniana é o seguinte teorema devido a Levi-Civita, cuja demonstração pode ser encontrada em [5].

Teorema 1.6 Dada uma variedade Riemanniana $M$, existe uma única conexão afim $\nabla$ em $M$ tal que:

(a) $\nabla$ é simétrica;

(b) $\nabla$ é compatível com a métrica de $M$.

A conexão $\nabla$ dada no teorema acima é dita a conexão Riemanniana (ou conexão de Levi-Civita) de $M$.

\subsection{A Segunda Forma Fundamental}

Sejam $M$ e $N$ variedades diferenciáveis de dimensão $n$ e $m$ respectivamente e $f: M \rightarrow N$ uma imersão. Suponha que $N$ tem uma métrica Riemanniana que indicaremos por $\tilde{g}$ e seja $\tilde{\nabla}$ a conexão Riemanniana de $N$. Então $f$ induz uma métrica Riemanniana em $M$ que será indicada por $g$. Seja $X$ um campo vetorial em $M$ e $U$ uma vizinhança de um ponto $x \in M$ onde $f$ é injetiva. Então $f_{*}(X)$ define um campo vetorial ao longo de $f(U)$ que pode ser estendido a um campo local $\tilde{X}$ numa vizinhança de $f(x)$ em $N$.

Lema 1.2 Sejam $X, Y$ campos vetoriais em $M$ e $\tilde{X}, \tilde{Y}$ extensões de $f_{*}(X)$ e $f_{*}(Y)$, respectivamente. Então a restrição de $\tilde{\nabla}_{\tilde{X}} \tilde{Y}$ a $f(M)$ não depende das extensões escolhidas.

A demostração deste lema pode ser encontrada em [22].

De acordo com o lema podemos denotar $\tilde{\nabla}_{\tilde{X}} \tilde{Y}$ por $\tilde{\nabla}_{X} Y$. 
Para cada $x \in M$, o produto interno em $T_{f(x)} N$ decompõe o mesmo na soma direta

$$
T_{f(x)} N=f_{*}\left(T_{x} M\right) \oplus\left(f_{*}\left(T_{x} M\right)\right)^{\perp}
$$

ou

$$
T_{x} N=T_{x} M \oplus T_{x} M^{\perp}
$$

onde $\left(T_{x} M\right)^{\perp}$ é o complemento ortogonal de $T_{x} M$ em $T_{x} N$. Assim dados os campos $X$ e $Y$ em $M$ temos a seguinte decomposição

$$
\tilde{\nabla}_{X} Y=T_{X} Y+h(X, Y)
$$

onde $T_{X} Y$ é a componente tangencial e $h(X, Y)$ é a componente normal. Nestas condiçôes temos o seguinte resultado.

Teorema 1.7 (a) $T_{X} Y=\nabla_{X} Y$, onde $\nabla$ é a conexão Riemanniana de $M$ com respeito à métrica induzida.

(b) A aplicação

$$
h: \mathcal{X}(M) \times \mathcal{X}(M) \rightarrow \mathcal{X}(M)^{\perp}
$$

é bilinear e simétrica sobre $\mathcal{D}(M)$.

\section{Demonstração :}

A prova baseia-se essencialmente nas propriedades definidoras da conexão Riemanniana $\tilde{\nabla}$. Sejam $X, Y, Z \in \mathcal{X}(M)$. Por um lado,

$$
\tilde{\nabla}_{X}(Z+W)=T_{X}(Z+W)+h(X, Z+W)
$$

e por outro lado, temos

$$
\tilde{\nabla}_{X}(Z+W)=\tilde{\nabla}_{X} Z+\tilde{\nabla}_{X} W=T_{X} Z+h(X, Z)+T_{X} W+h(X, W),
$$

e daí agrupando as componentes tangenciais e as componentes normais obtemos

$$
\begin{gathered}
T_{X}(Z+W)=T_{X} Z+T_{X} W, \\
h(X, Z+W)=h(X, Z)+h(X, W) .
\end{gathered}
$$

As outras propriedades de aditividade de $h$ e $T$ seguem das propriedades análogas da conexão $\tilde{\nabla}$. 
Se $\varphi \in \mathcal{D}(M)$, então

$$
\begin{aligned}
\tilde{\nabla}_{X}(\varphi Y) & =\varphi \tilde{\nabla}_{X} Y+X(\varphi) Y \\
& =\varphi T_{X} Y+\varphi h(X, Y)+X(\varphi) Y
\end{aligned}
$$

e por outro lado,

$$
\tilde{\nabla}_{X}(\varphi Y)=T_{X}(\varphi Y)+h(X, \varphi Y)
$$

Igualando as respectivas partes normais e tangenciais obtemos

$$
\begin{gathered}
h(X, \varphi Y)=\varphi h(X, Y), \\
T_{X}(\varphi Y)=\varphi T_{X} Y+X(\varphi) Y .
\end{gathered}
$$

Portanto, $h$ é bilinear e $T$ determina uma conexão afim em $M$. Vamos denotar a conexão $T$ por $\nabla$. Então podemos reescrever

$$
\tilde{\nabla}_{X} Y=\nabla_{X} Y+h(X, Y)
$$

Resta mostrar que $\nabla$ determina uma conexão Riemanniana em $M$. Ora, como $\tilde{\nabla}$ é simétrica temos

$$
\tilde{\nabla}_{X} Y-\tilde{\nabla}_{Y} X-[X, Y]=0
$$

ou seja,

$$
\nabla_{X} Y+h(X, Y)-\nabla_{Y} X-h(Y, X)-[X, Y]=0 .
$$

Igualando a zero as partes tangencial e normal obtemos

$$
\begin{gathered}
\nabla_{X} Y-\nabla_{Y} X-[X, Y]=0 \\
h(X, Y)=h(Y, X)
\end{gathered}
$$

ou seja, $\nabla$ e $h$ são simétricas. Além disso,

$$
\begin{aligned}
X g(Y, Z) & =X \tilde{g}(Y, Z) \\
& =\tilde{g}\left(\tilde{\nabla}_{X} Y, Z\right)+\tilde{g}\left(Y, \tilde{\nabla}_{X} Z\right) \\
& =\tilde{g}\left(\nabla_{X} Y+h(X, Y), Z\right)+\tilde{g}\left(Y, \nabla_{X} Z+h(X, Z)\right) \\
& =\tilde{g}\left(\nabla_{X} Y, Z\right)+\tilde{g}\left(Y, \nabla_{X} Z\right) \\
& =g\left(\nabla_{X} Y, Z\right)+g\left(Y, \nabla_{X} Z\right),
\end{aligned}
$$

provando assim que $\nabla$ é uma conexão compatível com a métrica de $M$. Portanto, $\nabla$ é a conexão Riemanniana de $M$ com respeito à métrica induzida. 
Definição 1.5 A aplicação $h$ definida no teorema acima é denominada a $2^{a}$ Forma Fundamental da Imersão $f$.

Seja $x \in M$ e $\xi \in\left(T_{x} M\right)^{\perp}$. Pelo teorema acima segue que a aplicação

$$
H_{\xi}: T_{x} M \times T_{x} M \rightarrow \mathbf{R}
$$

dada por

$$
H_{\xi}(u, v)=g(h(u, v), \xi)
$$

é uma forma bilinear e simétrica. A esta aplicação está associada uma aplicação linear auto-adjunta

$$
A_{\xi}: T_{x} M \rightarrow T_{x} M
$$

tal que

$$
g\left(A_{\xi}(u), v\right)=H_{\xi}(u, v)=g(h(u, v), \xi)
$$

Se $\xi$ é um campo normal a $M$, isto é, para cada $x \in M \xi(x) \in\left(T_{x} M\right)^{\perp}$ e, se $X \in \mathcal{X}(M)$, então, como antes o campo vetorial $\tilde{\nabla}_{X} \xi$ decompõe-se em uma parte tengencial, $\left(\tilde{\nabla}_{X} \xi\right)^{T}$, e em uma parte normal indicada por $\left(\tilde{\nabla}_{X} \xi\right)^{N}$. Nestas condições temos o seguinte resultado.

Proposição $1.1\left(\tilde{\nabla}_{X} \xi\right)^{T}=-A_{\xi} X$. Além disso, $\left(\tilde{\nabla}_{X} \xi\right)^{N}$ define uma conexáo afim em $M$, denominada a conexão normal de $M$.

\subsection{A aplicação de Gauss}

Seja $f: M^{n} \rightarrow \mathbf{E}^{m}$ uma imersão da $n$-variedade $M$ no espaço euclideano $\mathbf{E}^{m}$. Consideremos o fibrado normal unitário de $f(M)$ dado por

$$
B=\left\{(x, v) \in M \times \mathbf{E}^{m} ;\|v\|=1, v \in\left(T_{x} M\right)^{\perp}\right\} .
$$

Claramente, $B$ é uma variedade diferenciável de dimensão $m-1$ cujo espaço base é $M$ e cada fibra é uma esfera $S^{m-n-1}$. A aplicação

$$
\nu: B \rightarrow S_{0}^{m-n-1}
$$

de $B$ na esfera unitária $S^{m-1}$ centrada na origem de $\mathbf{E}^{m}$ definida por

$$
\nu(x, v)=v
$$


é dita a Aplicação de Gauss da imersão $f$. Geometricamente, para cada $(x, v) \in B, \nu(x, v)$ é o ponto final do transladado de $v$ para a origem de $\mathbf{E}^{m}$.

No que segue, relacionaremos as singularidades não degeneradas das funções altura com os valores regulares da Aplicação de Gauss $\nu$. O resultado central neste contexto é o seguinte teorema.

Teorema 1.8 A nulidade da aplicação de Gauss $\nu$ em um ponto $\xi=(x, v) \in B$ é igual à nulidade de $A_{v}$. Em particular, $\xi$ é ponto crítico de $\nu$ se, e somente se, $A_{v}$ é singular.

\section{Demonstração:}

Seja $\left(u_{1}, \ldots, u_{n}\right)$ um sistema de coordenadas em uma vizinhança $U$ de $x$ na qual $f(x)=0$ e $v=(0,0, \ldots, 1) \in \mathbf{E}^{m}$. Neste sistema, escrevendo

$$
f\left(u_{1}, \ldots, u_{n}\right)=\left(f_{1}, \ldots, f_{m}\right)
$$

podemos identificar os vetores

$$
\frac{\partial f}{\partial u_{i}}=f_{*}\left(\frac{\partial}{\partial u_{i}}\right)=(0, \ldots, 1, \ldots, 0), \quad i=1, \ldots, n .
$$

Escolhamos $m-n$ campos normais unitários

$$
\eta_{1}\left(u_{1}, \ldots, u_{n}\right), \ldots, \eta_{m-n}\left(u_{1}, \ldots, u_{n}\right)
$$

ao longo de $U$, dois a dois ortogonais. Então, neste sistema a aplicação de Gauss é dada pela expressão

$$
\nu\left(u_{1}, \ldots, u_{n}, t_{1}, \ldots, t_{m-n-1}\right)=\sum_{i=1}^{m-n} t_{i} \eta_{i}\left(u_{1}, \ldots, u_{n}\right)
$$

onde

$$
t_{m-n}=\sqrt{1-\sum_{i=1}^{m-n-1} t_{i}^{2}}
$$

Suas derivadas parciais são dadas por:

$$
\left\{\begin{array}{l}
\frac{\partial \nu}{\partial u_{j}}(\xi)=\sum_{i=1}^{m-n} t_{i} \frac{\partial \eta_{i}}{\partial u_{j}} \\
\frac{\partial \nu}{\partial t_{k}}(\xi)=\eta_{k}(x)+\frac{\partial}{\partial t_{k}}\left(\sqrt{1-\sum t_{i}^{2}}\right) \eta_{m-n}(x), k=1, \ldots, m-n-1
\end{array}\right.
$$


Uma base para $T_{\xi} B \sim T_{v} S^{m-1}$ é dada por

$$
\frac{\partial f}{\partial u_{1}}(x), \ldots, \frac{\partial f}{\partial u_{n}}(x), \eta_{1}(x), \ldots, \eta_{m-n-1}(x),
$$

e nesta base $\nu_{*}$ é representada pela matriz $(m-1) \times(m-1)$

$$
\left(\begin{array}{ccc}
\left(\sum_{i=1}^{m-n} t_{i} g\left(\frac{\partial \eta_{i}}{\partial u_{j}}(x), \frac{\partial f}{\partial u_{k}}(x)\right)\right) & \vdots & \left(\sum_{i=1}^{m-n} t_{i} g\left(\frac{\partial \eta_{i}}{\partial u_{j}}(x), \eta_{k}(x)\right)\right) \\
0 & \vdots & I
\end{array}\right)
$$

Por outro lado temos,

$$
0=\frac{\partial}{\partial u_{j}} g\left(\eta_{i}, \frac{\partial f}{\partial u_{k}}\right)=g\left(\frac{\partial \eta_{i}}{\partial u_{j}}, \frac{\partial f}{\partial u_{k}}\right)+g\left(\eta_{i}, \frac{\partial^{2} f}{\partial u_{j} \partial u_{k}}\right)
$$

Logo, a matriz acima fica

$$
\left(\begin{array}{ccc}
\left(-\sum_{i=1}^{m-n} t_{i} g\left(\eta_{i}, \frac{\partial^{2} f}{\partial u_{j} \partial u_{k}}\right)(x)\right) & \vdots & \left(\sum_{i=1}^{m-n} t_{i} g\left(\frac{\partial \eta_{i}}{\partial u_{j}}(x), \eta_{k}(x)\right)\right) \\
0 & \vdots & I
\end{array}\right)
$$

Deste modo a nulidade de $\nu$ em $\xi$ é igual à nulidade da matriz $n \times n$

$$
\left(\sum_{i=1}^{m-n} t_{i} g\left(\eta_{i}, \frac{\partial^{2} f}{\partial u_{j} \partial u_{k}}\right)(x)\right)
$$

que é a matriz de $A_{v}$. Segue também que $\operatorname{det}\left(\nu_{*}\right)=(-1)^{n} \operatorname{det}\left(A_{v}\right)$.

Para cada $p \in S^{m-1}$, definimos a função $\ell_{p}: \mathbf{E}^{m} \rightarrow \mathbf{R}$ por

$$
\ell_{p}(q)=<p, q>
$$

que é dita a função altura linear com relação a $p$. Restringindo $\ell_{p}$ a $M$ obtemos uma função em $M$ a valores reais dada por

$$
\ell_{p}(x)=<p, f(x)>.
$$

Geometricamente, $\ell_{p}(x)$ representa a altura do ponto $f(x)$ em relação ao vetor unitário $p$. Os resultados a seguir relacionam os pontos críticos não degenerados das funções altura com os valores regulares da aplicação de Gauss $\nu$. 
Teorema 1.9 Seja $f: M^{n} \rightarrow \mathbf{E}^{m}$ uma imersão e $p \in S^{m-1}$. Então:

(a) $\ell_{p}$ tem um ponto crítico em $x \in M$ se, e somente se, $p \in\left(T_{x} M\right)^{\perp}$.

(b) se $\ell_{p}$ tem um ponto crítico em $x \in M$, então

$$
H_{x}(u, v)=\left\langle A_{p}(u), v\right\rangle, \forall u, v \in T_{x} M .
$$

\section{Demonstração :}

(a) Dado $x \in M$ seja $U$ uma vizinhança mergulhada de $x$. Seja $u \in T_{x} M$ e $\gamma(t)$ uma curva diferenciável em $U \operatorname{com} \gamma(0)=x$ e $\frac{d \gamma}{d t}(0)=u$. Então,

$$
\left(\ell_{p}\right)_{*_{x}}(u)=\frac{d}{d t}\left(\ell_{p} \circ \gamma(t)\right)_{\left.\right|_{t=0}}=\frac{d}{d t}\langle\gamma(t), p\rangle_{\left.\right|_{t=0}}=\langle u, p\rangle
$$

donde $\left(\ell_{p}\right)_{*_{x}}(u)=0$ se, e somente se, $\langle u, p\rangle=0$. Portanto $x$ é ponto crítico de $\ell_{p}$ se, e somente se $p \in\left(T_{x} M\right)^{\perp}$.

(b) Sejam $u, v \in T_{x} M$ e $V$ uma extensão de $v$ a um campo local em U. Então

$$
\begin{aligned}
H_{x}(u, v) & =u\left(\ell_{p}\right)(x) \\
& =u<p, v>(x) \\
& =\left\langle p, \tilde{\nabla}_{u} V>\right.
\end{aligned}
$$

Por outro lado, se $\xi$ é um campo de vetores normais em $U$ com $\xi_{x}=p$, então $<V, \xi>=0$ e, portanto,

$$
\begin{aligned}
0 & =u<V, \xi> \\
& =<\tilde{\nabla}_{u} V, \xi>+<V, \tilde{\nabla}_{u} \xi> \\
& =<\tilde{\nabla}_{u} V, \xi>+<V,-A_{\xi}(u)+\frac{1}{\nabla_{u}} \xi> \\
& =<\tilde{\nabla}_{u} V, \xi>-<V, A_{\xi}(u)>.
\end{aligned}
$$

Assim em $x$, temos

$$
<\tilde{\nabla}_{u} V, p>=<A_{p}(u), v>
$$

e pela equação 1.1 segue que

$$
H_{x}(u, v)=<A_{p}(u), v>.
$$

Um corolário imediato é o seguinte: 
Corolário 1.3 Seja $p \in\left(T_{x} M\right)^{\perp}$. Então:

(a) $\ell_{p}$ tem um ponto crítico degenerado em $x$ se, e somente se, $A_{p}$ é singular.

(b) $S e \ell_{p}$ tem um ponto crítico não-degenerado em $x$, então o índice de $\ell_{p}$ em $x$ é igual ao número de autovalores negativos de $A_{p}$.

Chegamos agora ao ponto central deste parágrafo. A seguir, combinando os resultados anteriores, vamos dar o prometido relacionamento entre a aplicação de Gauss e as funções altura.

Teorema 1.10 Para $p \in S^{m-1}, \ell_{p}$ é uma função de Morse se, e somente se, $p$ é um valor regular da aplica̧̧ão de Gauss $\nu$.

Corolário 1.4 (a) Para quase todo $p \in S^{m-1}, \ell_{p}$ é uma função de Morse.

(b) Suponha que $\ell_{p}$ tem um ponto crítico náo degenerado de indice $j$ em $x \in M$. Então existe uma função de Morse $\ell_{q}$ com um ponto crítico $y \in M$ de índice $j$ (podemos escolher $q$ e y próximos de $p$ e $x$, respectivamente).

\section{Demonstração :}

(a) Considere o conjunto $\mathcal{C}$ de pontos críticos da aplicação de Gauss $\nu: B \rightarrow S^{m-1}$. Pelo teorema de Sard, $\nu(\mathcal{C})$ tem medida nula em $S^{m-1}$. Portanto pelo teorema anterior segue que $\ell_{p}$ é um função de Morse para quase todo $p \in S^{m-1}$.

(b) Pela parte (a) do teorema 1.9, $p \in\left(T_{x} M\right)^{\perp}$ e portanto, $p=\nu(\xi)$, onde $\xi \in B$ é tal que $\pi(\xi)=x$ e $\pi: B \rightarrow M$ é a projeção canônica. Como $\ell_{p}$ tem um ponto crítico não degenerado de índice $\mathrm{j}$ em $x$, segue que $\nu_{*}$ é não-singular em $x$ e $A_{\xi}$ tem exatamente $\mathrm{j}$ autovalores negativos e $\mathrm{n}-\mathrm{j}$ autovalores positivos. Pelo teorema da função inversa $\nu$ é um difeomorfismo de uma vizinhança $V$ de $\xi=(x, \nu(x))$ em $B$ sobre uma vizinhança $U$ de $p$ em $S^{m-1}$. Seja $q \in U$ um valor regular de $\nu$. Então pelo terema 1.10, $\ell_{q}$ é uma função de Morse e tem um ponto crítico $y \in \pi(V)$. Além disso, como $\nu_{*}$ é não-singular em $V$, o número de autovalores negativos de $A_{\eta}$ permanece constante quando $\eta$ percorre $V$. Então o índice de $\ell_{q}$ em $y$ também é igual a j. 


\section{Capítulo 2}

\section{Aplicações Justas}

Neste capitulo consideraremos imersões $f: M^{n} \rightarrow \mathbf{E}^{m}$ de variedades Riemannianas compactas e conexas. Definiremos uma curvatura, induzida pela imersão, denominada curvatura de Lipschitz-Killing, que generaliza a curvatura Gaussiana de hipersuperfícies. Quando integrada sobre o fibrado normal unitário, obtem-se uma nova medida de curvatura, denominada curvatura total, que dá informações sobre a topologia da variedade. A integral do módulo da curvatura de LipschitzKilling tomada sobre o fibrado normal esférico, fornece a curvatura total absoluta da variedade. Com a ajuda da Teoria de Morse obtem-se uma expressão simples para esta medida de curvatura em termos de pontos críticos de funções altura e, com esta expressão podemos concluir parte de um resultado clássico da Geometria Diferencial, o bem conhecido Teorema de Chern-Lashof. Estamos particularmente interessados por aquelas imersões cuja curvatura total absoluta assume o valor mínimo possível. Tais imersões são ditas de curvatura total absoluta mínima. Uma segunda etapa é introduzir a noção de justeza, devida a Kuiper, e mostrar que, com uma hipótese adicional, esta noção é equivalente a curvatura total absoluta mínima.

\subsection{Curvatura Total Absoluta}

Seja $f: M^{n} \rightarrow \mathbf{E}^{m}$ uma imersão diferenciável definida na variedade compacta e conexa $M$. Seja $B=\left\{(x, v) \in M \times \mathbf{E}^{m} ; v \in T_{x} M^{\perp} e\|v\|=1\right\}$ o fibrado normal unitário de $f(M)$. Indiquemos por $d a$ o elemento de volume de $S^{m-1}$ e por $d M$ o elemento de volume de $M$ e consideremos em $B$ a forma diferencial $d \sigma$ de grau $m-n-1$ tal que sua restrição a uma fibra é o elemento de volume da esfera 
de vetores normais unitários em um ponto $p \in M$. Então $d A=d \sigma \wedge d M$ é o elemento de volume de $B$.

Definição 2.1 A aplicação $G: B \rightarrow \mathbf{R}$ definida pela equação

$$
\nu^{*} d a(x, v)=G(x, v) d A
$$

é dita a curvatura de Lipschitz-Killing, onde $\nu$ é a aplicação de Gauss e $\nu^{*}$ é a aplicação dual de $\nu_{*}: T_{x} B \rightarrow T_{v} S^{m-1}$.

Vamos obter uma expressão simples para $G$ em um sistema de coordenadas. Dado $(x, v) \in B$ consideremos $\left(u_{1}, \ldots, u_{n}\right)$ um sistema de coordenadas em uma vizinhança $U$ de $x$ e escolhamos $\eta_{1}, \ldots, \eta_{m-n-1}$ campos normais unitários dois a dois ortogonais ao longo de $U$. Então o conjunto

$$
\left\{e_{1}, \ldots, e_{n}, e_{n+1}, \ldots, e_{m-1}\right\}
$$

é uma base de $T_{(x, v)} B$, onde

$$
e_{i}=\frac{\partial}{\partial u_{i}}, i=1, \ldots, n \text { e } e_{n+j}=\eta_{j}, j=1, \ldots, m-n-1 .
$$

Podemos supor que esta base seja ortonormal. Seja $\left\{d e_{1}, \ldots, d e_{m-1}\right\}$ a sua base dual. Assim, temos

$$
\nu^{*} d a(x, v)\left(e_{1}, \ldots, e_{m-1}\right)=d A(\nu(x, v))\left(\nu_{*}\left(e_{1}\right), \ldots, \nu_{*}\left(e_{m-1}\right)\right) .
$$

Por outro lado, como $T_{(x, v)} B \sim T_{\nu(x, v)} S^{m-1}$, temos

$$
d A(\nu(x, v))=d e_{1} \wedge \cdots \wedge d e_{m-1}
$$

e portanto

$$
\begin{aligned}
\nu^{*} d a(x, v)\left(e_{1}, \ldots, e_{m-1}\right) & =d e_{1} \wedge \cdots \wedge d e_{m-1}\left(\nu_{*}\left(e_{1}\right), \ldots, \nu_{*}\left(e_{m-1}\right)\right) \\
& =\operatorname{det}\left(\operatorname{de}_{i}\left(\nu_{*}\left(e_{j}\right)\right)\right) .
\end{aligned}
$$

Ora, sendo $\left\{e_{1}, \ldots, e_{m-1}\right\}$ base ortonormal, temos

$$
\nu_{*}\left(e_{j}\right)=\frac{\partial \nu}{\partial e_{j}}=\sum_{k=1}^{m-1}<\frac{\partial \nu}{\partial e_{j}}, e_{k}>e_{k}
$$

e portanto,

$$
\begin{aligned}
d e_{i}\left(\nu_{*}\left(e_{j}\right)\right) & =\sum_{k=1}^{m-1}<\frac{\partial \nu}{\partial e_{j}}, e_{k}>d e_{i}\left(e_{k}\right) \\
& =<\frac{\partial \nu}{\partial e_{j}}, e_{i}>
\end{aligned}
$$


Logo,

$$
\begin{aligned}
\nu^{*} d a(x, v)\left(e_{1}, \ldots, e_{m-1}\right) & =\operatorname{det}\left(\begin{array}{ll}
-A_{v} & * \\
0 & I
\end{array}\right) \\
& =(-1)^{n} \operatorname{det}\left(A_{v}\right) .
\end{aligned}
$$

Pela definição 2.1 temos

$$
\begin{aligned}
\nu^{*} d a(x, v) & =G(x, v) d A \\
& =G(x, v) d e_{1} \wedge \cdots \wedge d e_{m-1}
\end{aligned}
$$

e portanto,

$$
G(x, v)=(-1)^{n} \operatorname{det}\left(A_{v}\right) .
$$

Desta expressão e do teorema 1.8 , segue que $G(x, v)=0$ se, e somente se, $(x, v)$ é ponto crítico da aplicação de Gauss $\nu$.

Definição 2.2 A integral

$$
K(x)=\int|G(x, v)| d \sigma
$$

tomada sobre a esfera de vetores normais unitários em $x \in M$ é dita a curvatura total absoluta de $M$ em x e, a curvatura total absoluta de $M$ é dada pela integral

$$
\tau(M, f)=\int_{M} K(x) d M
$$

se esta converge.

Segue da definição que

$$
\begin{aligned}
\tau(M, f) & =\int_{M} K(x) d M \\
& =\int_{M} \int_{S^{m-1}}|G(x, v)| d \sigma \wedge d M \\
& =\int_{B}|G(x, v)| d A .
\end{aligned}
$$

Seja $p \in S^{m-1}$ um valor regular da aplicação de Gauss $\nu$ e $\mu(p)$ o número de pontos críticos da função de Morse $\ell_{p}$. Considere uma família $\left\{U_{\lambda}\right\}_{\lambda \in L}$ de abertos conexos disjuntos do fibrado normal unitário $B$ tal que, exceto por um conjunto de medida nula, a união $\bigcup_{\lambda \in L} U_{\lambda}$ é igual ao conjunto dos pontos regulares de $\nu$ 
com a condição de que $\nu$ seja injetiva em cada $U_{\lambda}$. Suponhamos, daqui por diante, que o elemento de volume de $S^{m-1}$ está normalizado, isto é,

$$
\int_{S^{m-1}} d a=1
$$

Pelo Teorema de Mudança de Variáveis,

$$
\begin{aligned}
\operatorname{Area}\left(\nu\left(U_{\lambda}\right)\right) & =\int_{\nu\left(U_{\lambda}\right)} 1 d a \\
& =\int_{U_{\lambda}} 1 \nu^{*} d a \\
& =\int_{U_{\lambda}}\left|\operatorname{det}\left(\nu_{*}\right)\right| d A \\
& =\int_{U_{\lambda}}|G| d A .
\end{aligned}
$$

Seja $\mu\left(U_{\lambda}, v\right)$ o número de pontos críticos da função altura $\ell_{v}$ em $\pi\left(U_{\lambda}\right)$, onde $\pi: B \rightarrow M$ é a projeção canônica. Ora, como $\nu(x, v)=v$ e $\nu$ é injetiva em $U_{\lambda}$, segue que $\mu\left(U_{\lambda}, v\right)=1, \forall v \in \nu\left(U_{\lambda}\right)$ e, além disso, $\mu\left(U_{\lambda}, q\right)=0$ se $q \notin \nu\left(U_{\lambda}\right)$. Assim,

$$
\begin{aligned}
\int_{U_{\lambda}}\left|\operatorname{det}\left(\nu_{*}\right)\right| d A & =\int_{\nu\left(U_{\lambda}\right)} 1 d a \\
& =\int_{\nu\left(U_{\lambda}\right) \cap D} 1 d a \\
& =\int_{\nu\left(U_{\lambda}\right) \cap D} \mu\left(U_{\lambda}, \cdot\right) d a \\
& =\int_{D} \mu\left(U_{\lambda}, \cdot\right) d a
\end{aligned}
$$

onde $D=S^{m-1}-\mathcal{C}$ e $\mathcal{C}$ é o conjunto dos valores críticos da aplicação de Gauss $\nu$. Portanto a curvatura total absoluta é dada por

$$
\begin{aligned}
\tau(M, f) & =\int_{B}|G| d A \\
& =\int_{B}\left|\operatorname{det}\left(\nu_{*}\right)\right| d A \\
& \left.=\int_{U U_{\lambda}} \mid \operatorname{det}\left(\nu_{*}\right)\right) \mid d A \\
& =\sum_{\lambda \in L} \int_{U_{\lambda}}\left|\operatorname{det}\left(\nu_{*}\right)\right| d A \\
& =\sum_{\lambda \in L} \int_{D} \mu\left(U_{\lambda}, \cdot\right) d a .
\end{aligned}
$$


Observe que basta integrar sobre a união disjunta $\bigcup U_{\lambda}$ pois $\operatorname{det}\left(\nu_{*}(x, v)\right)=0$ se $(x, v) \notin \bigcup U_{\lambda}$. Além disso, se $p \in S^{m-1}-\mathcal{C}$, então

$$
\mu(p)=\sum_{\lambda} \mu\left(U_{\lambda}, p\right)
$$

e este número é finito pois os pontos críticos de $\ell_{p}$ são isolados e $M$ é compacta. Também $\mu\left(U_{\lambda}, p\right)=0$ exceto para um número finito de índices $\lambda_{i} \in L$. Logo,

$$
\begin{aligned}
\tau(M, f) & =\sum_{\lambda_{i}} \int_{D} \mu\left(U_{\lambda_{i}}, \cdot\right) d a \\
& =\int_{p \in D} \mu(p) d a
\end{aligned}
$$

Seja $\gamma(M)$ o número de Morse de $M$, isto é,

$$
\gamma(M)=\min \{\mu(\phi) ; \phi: M \rightarrow \mathbf{R} \text { é de Morse em } M\}
$$

onde $\mu(\phi)$ é o número de pontos críticos de $\phi$. Ora, para cada função de Morse $\ell_{p}$ em $M$, temos $\mu(p) \geq \gamma(M)$ e, portanto

$$
\tau(M, f) \geq \gamma(M)
$$

Mostraremos a seguir que $\gamma(M)$ é, de fato, o menor valor possivel para $\tau(M, f)$ quando $f$ percorre o conjunto das imersões de $M$ em espaços euclideanos. Este é o conteúdo do seguinte teorema.

Teorema 2.1 Seja $M$ uma variedade compacta. O ínfimo do conjunto

$$
\left\{\tau(M, f) ; f: M \rightarrow \mathbf{E}^{m} \text { é imersão em espaços euclideanos }\right\}
$$

é igual a $\gamma(M)$.

\section{Demonstração:}

Seja $f: M \rightarrow \mathbf{E}^{m}$ uma imersão e $\phi: M \rightarrow \mathbf{R}$ uma função de Morse em $M$ com $\gamma(M)$ pontos críticos. Para cada $\lambda \in(0, \infty)$ defina a aplicação $h_{\lambda}: M \rightarrow \mathbf{E}^{m+1}$ por

$$
h_{\lambda}(x)=(f(x), \lambda \phi(x)) .
$$

$h_{\lambda}$ é claramente uma imersão, pois $\operatorname{rank}\left(h_{\lambda}\right) \geq \operatorname{rank}(f)=\operatorname{dim} M$. Para mostrarmos que inf $\tau(M, f)=\gamma(M)$, dado $\epsilon>0$ devemos exibir uma imersão $h$ tal que 
$\tau(M, h)<\epsilon+\gamma(M)$. Então basta mostrarmos que $\lim _{\lambda \rightarrow \infty} \tau\left(M, h_{\lambda}\right)=\gamma(M)$. Seja $p=(0,0, \ldots, 1) \in \mathbf{E}^{m+1}$. Então,

$$
\ell_{p}\left(h_{\lambda}(x)\right)=<p, h_{\lambda}(x)>=\lambda \phi(x),
$$

e portanto $\ell_{p}$ é não degenerada e tem $\gamma(M)$ pontos críticos. A idéia da prova é que quando $\lambda$ cresce a imersão $h_{\lambda}$ alonga-se na direção de $p$, e com isto mais e mais funções altura tem o mesmo comportamento que $\phi$ nos pontos críticos, isto é, tem $\gamma(M)$ pontos críticos. Então a integral de $\mu(q)$ sobre a esfera $S^{m}$ em $\mathbf{E}^{m+1}$ torna-se arbitrariamente próxima de $\gamma(M)$, isto é,

$$
\lim _{\lambda \rightarrow \infty} \tau\left(M, h_{\lambda}\right)=\gamma(M)
$$

Formalizemos então os detalhes da prova. Começamos analisando a imersão $h_{1}$. Como $p$ é valor regular da aplicação de Gauss de $h_{1}$, existe uma vizinhança $U=B(p, \epsilon)$ de $p$ em $S^{m}$ tal que se $q \in U$, então $\ell_{q} \circ h_{1}$ é não degenerada e tem $\gamma(M)$ pontos críticos em $M$. Então, se tomarmos $q=w+p$, onde $w \in \mathbf{E}^{m} \mathbf{e}$ $|w|^{2}<\epsilon$, temos que

$$
\ell_{q}\left(h_{1}(x)\right)=<w, f(x)>+\phi(x),
$$

é não degenerada e tem $\gamma(M)$ pontos críticos. Segue que, para $\lambda \in(0, \infty)$ e $w=\lambda u, \operatorname{com} u \in \mathbf{E}^{m}$,

$$
\ell_{q}\left(h_{\lambda}(x)\right)=<\lambda u, f(x)>+\lambda \phi(x)=\lambda(<u, f(x)>+\phi(x)) .
$$

Então $\ell_{q} \circ h_{\lambda}$ é não degenerada e tem $\gamma(M)$ pontos críticos para $|u|^{2}<\epsilon$. Suponha agora que $q=w+\beta p \in S^{m} \operatorname{com} \beta \neq 0$. Então $\ell_{q}$ tem o mesmo comportamento crítico que $\ell_{q^{\prime}}$ onde $q^{\prime}=(w / \beta)+p$. Então $\ell_{q}$ é não degenerada em $h_{\lambda}(M)$ e tem $\gamma(M)$ pontos críticos se $\left|\frac{w}{\beta}\right|^{2}<\lambda^{2} \epsilon$. Observando que $|w|^{2}=1-\beta^{2}$, podemos escrever

$$
\mu_{\lambda}(q)=\gamma(M), \text { se }|\beta|>\left(1+\lambda^{2} \epsilon\right)^{-1 / 2}
$$

onde $\mu_{\lambda}(q)$ é o número de pontos críticos de $\ell_{q}$ em $h_{\lambda}(M)$. Seja $\alpha=\left(1+\lambda^{2} \epsilon\right)^{-1 / 2}$ e considere o conjunto

$$
W_{\lambda}=\left\{\mathbf{E}^{m} \times[-\alpha, \alpha]\right\} \cap S^{m}
$$

Então, se $q \in S^{m}-W_{\lambda}$, temos

$$
\mu_{\lambda}(q)=\gamma(M)
$$

Agora,

$$
\tau\left(M, h_{\lambda}\right)=\int_{S^{m}-W_{\lambda}} \mu_{\lambda}(q) d a+\int_{W_{\lambda}} \mu_{\lambda}(q) d a
$$


Note que, quando $\lambda \rightarrow \infty$, o intervalo $[-\alpha, \alpha]$ tende ao conjunto $\{0\}$ e, portanto $W_{\lambda}$ tende ao conjunto $\left(\mathbf{E}^{m} \times\{0\}\right) \cap S^{m}=S^{m-1}$, o qual tem medida nula em $S^{m}$. Assim,

$$
\begin{aligned}
\lim _{\lambda \rightarrow \infty} \int_{S^{m}-W_{\lambda}} \mu_{\lambda}(q) d a & =\lim _{\lambda \rightarrow \infty} \int_{S^{m}-W_{\lambda}} \gamma(M) d a \\
& =\int_{S^{m}-S^{m-1}} \gamma(M) d a \\
& =\gamma(M) .
\end{aligned}
$$

Da equação 2.3 o teorema é consequência do seguinte lema.

Lema 2.1 (Wilson) $S e j a I_{\lambda}=\int_{W_{\lambda}} \mu_{\lambda}(q) d a$. Então

$$
\lim _{\lambda \rightarrow \infty} I_{\lambda}=0 \text {. }
$$

A demonstração deste lema encontra-se em [23].

Definição 2.3 Uma imersão $f: M \rightarrow \mathbf{E}^{m}$ é dita de curvatura total absoluta mínima se $\tau(M, f)=\gamma(M)$.

Teorema 2.2 Uma imersão $f: M \rightarrow \mathbf{E}^{m}$ tem curvatura total absoluta minima se, e somente se, toda função altura não degenerada tem $\gamma(M)$ pontos críticos.

\section{Demonstração:}

Como $\tau(M, f)=\int_{S^{m-1}-\mathcal{C}} \mu(p) d a$, se $\mu(p)=\gamma(M)$ para todo $p \in S^{m-1}$ tal que $\ell_{p}$ é não degenerada, então $\tau(M, f)=\gamma(M)$.

Reciprocamente, suponha que $\mu(q)>\gamma(M)$ para uma dada $\ell_{q}$ não degenerada. Então, pelo corolário 1.4 do teorema 1.10, existe uma vizinhança $U$ de $q$ em $S^{m-1}$ tal que para todo $p \in U, \ell_{p}$ é não degenerada e $\mu(p) \geq \mu(q)$. Logo,

$$
\int_{p \in U} \mu(p) d a>\gamma(M),
$$

e portanto, $\tau(M, f)>\gamma(M)$. 


\subsection{Hipersuperfícies Convexas}

Um primeiro exemplo de imersões com curvatura total absoluta mínima são as hipersuperfícies convexas. Neste sentido, diz-se que a noção de curvatura total absoluta mínima generaliza a noção de convexidade.

Diremos que a fronteira de um conjunto convexo m-dimensional em $\mathbf{E}^{m}$ é uma hipersuperfície convexa em $\mathbf{E}^{m}$. Formulemos este conceito em termos de planos suportes, como é feito na Geometria Diferencial.

Definição 2.4 Seja $V$ um subconjunto de $\mathbf{E}^{m}$. Um hiperplano $\Phi$ em $\mathbf{E}^{m}$ por um ponto $x \in V$ é dito um plano suporte de $V$ em $x$ se $V$ está inteiramente contido em um dos semi-espaços fechados determinado por $\Phi$. Tal semi-espaço é dito um semi-espaço suporte.

Definição 2.5 Um mergulho diferenciável $f: M^{n} \rightarrow \mathbf{E}^{n+1}$ é dito uma hipersuperfície convexa se para cada $x \in M$, o hiperplano tangente a $f(M)$ em $f(x)$ é um plano suporte de $f(M)$.

Definição 2.6 Seja $V$ um subconjunto de $\mathbf{E}^{m}$. O fecho convexo de $V$, denotado por $\mathcal{H} V$, é o menor convexo fechado que contém $V$.

O seguinte teorema relaciona as duas definições de hipersuperfície convexa.

Teorema 2.3 Seja $f: M^{n} \rightarrow \mathbf{E}^{n+1}$ uma imersão da variedade compacta $M$. Então $f$ mergulha $M$ sobre $\partial \mathcal{H} f(M)$ se, e somente se, todo plano tangente a $f(M)$ é um plano suporte de $f(M)$.

Uma demonstração deste resultado pode ser encontrada em [6]. Mostraremos a. seguir que, se $f: S^{n} \rightarrow \mathbf{E}^{n+1}$ é uma hipersuperfície convexa então $f$ mergulha $S^{n}$ com curvatura total absoluta mínima.

Teorema 2.4 Se $f: S^{n} \rightarrow \mathbf{E}^{n+1}$ é uma hipersuperfície convexa compacta, então $\tau\left(S^{n}, f\right)=2$, isto é, $f$ mergulha $S^{n}$ com curvatura total absoluta minima.

\section{Demonstração:}


Suponha que $\tau\left(S^{n}, f\right)>2$. Então existe $\ell_{p}$ não degenerada com pelo menos três pontos críticos $x_{1}, x_{2}, x_{3} \in S^{n}$. Os hiperplanos tangentes nestes pontos são todos paralelos e são planos suporte de $f\left(S^{n}\right)$. Se $\ell_{p}$ assumir três valores distintos em $x_{1}, x_{2}, x_{3}$ então um destes planos não será plano suporte, contrariando a convexidade. Por outro lado, se $\ell_{p}\left(x_{i}\right)=\ell_{p}\left(x_{j}\right)$ com $i \neq j$, então os planos tangentes coincidem. Seja $\Phi$ tal plano. A convexidade de $\mathcal{H} f\left(S^{n}\right)$ implica que o segmento $\left[x_{i}, x_{j}\right]$ está contido em $\mathcal{H} f\left(S^{n}\right)$. Ora, $f\left(S^{n}\right)=\partial \mathcal{H} f\left(S^{n}\right)$ pois $f$ é hipersuperfície convexa. Então, como $\left[x_{i}, x_{j}\right] \subset \Phi$ e $\Phi$ é plano suporte segue que $\left[x_{i}, x_{j}\right] \subset \partial \mathcal{H} f\left(S^{n}\right)=f\left(S^{n}\right)$. Concluimos que $x_{i}$ e $x_{j}$ são pontos críticos não isolados de $\ell_{p}$, contrariando o fato de ser $\ell_{p}$ não degenerada.

A recíproca deste teorema é verdadeira e foi provada em 1957 por ChernLashof [8] para imersóes diferenciáveis. Em 1970, Kuiper [12] provou o mesmo resultado para imersões topológicas. Esta prova será dada mais adiante após estarmos familiarizados com a noção de top-sets. A seguir demonstramos um resultado devido a Chern-Lashof [8].

Teorema 2.5 (Chern-Lashof) Seja $f: M \rightarrow \mathbf{E}^{m}$ uma imersão de uma variedade compacta n-dimensional. Então,

(a) $\tau(M, f) \geq 2$;

(b) Se $\tau(M, f)<3, M$ é homeomorfa a uma n-esfera.

\section{Demonstração:}

(a)Toda função altura não degenerada $\ell_{p}$ tem um máximo e um mínimo em $M$, donde $\mu(p) \geq 2$ e, portanto $\tau(M, f)=\int_{S^{m-1}} \mu(p) d a \geq 2$.

(b)Se $\tau(M, f)<3$, existe um conjunto $U$ em $S^{m-1}$ de medida positiva tal que $\mu(p)=2 \mathrm{em} U$. Então existe uma $\ell_{p}$ não degenerada com exatamente dois pontos críticos e, portanto pelo teorema de Reeb 1.4, $M$ é homeomorfa a $S^{n}$.

\subsection{Aplicações Justas e Imersões}

Introduziremos agora o conceito de justeza que veremos será equivalente a curvatura total absoluta mínima para imersões que satisfazem a condição $\gamma(M)=$ $\sum_{i=1}^{n} \beta_{i}\left(M ; \mathbf{Z}_{2}\right)$ onde $\beta_{i}$ é o i-ésimo número de Betti de $M$ com coeficientes em $\mathbf{Z}_{2}$. A formulação de justeza é devida a Kuiper [13] e envolve interseç̧ões de semi-espaços e injetividade em homologia, o que torna justeza uma propriedade invariante por transformações projetivas e ortogonais. A condição acima mencionada foi introduzida por Kuiper como 
Condiçâo 3A: $\mathrm{O}$ número de Morse $\gamma(M)$ é igual à soma dos números de Betti, $\beta(M)=\sum \beta_{i}\left(M ; \mathbf{Z}_{2}\right)$.

Antes de formalizarmos a definição demonstremos um lema que a motivará.

Lema 2.2 Seja $\phi: M \rightarrow \mathbf{R}$ uma função de Morse definida na variedade compacta $M$. Então $\mu(\phi)=\beta\left(M ; \mathbf{Z}_{2}\right)$ se, e somente se, para todo $r \in \mathbf{R}$ a inclusão $M_{r}(\phi) \hookrightarrow M$ induz monomorfismo em homologia.

\section{Demonstração:}

Com as mesmas notações da demonstração das desigualdades de Morse (teorema 1.5), temos

$$
\sum \mu_{\lambda}(\phi)-\sum \beta_{\lambda}\left(M ; \mathbf{z}_{2}\right)=2 \sum_{\lambda=0}^{n-1} b_{\lambda}+b_{n},
$$

onde $b_{\lambda}=\sum b_{\lambda}^{i}$ e $b_{\lambda}^{i}=\operatorname{rank}\left(\partial_{\lambda+1}\right)$. Suponhamos então que $\mu(\phi)=\beta\left(M ; \mathbf{Z}_{2}\right)=$ $\sum \beta_{\lambda}\left(M ; Z_{2}\right)$. Portanto, da equação 2.4 , segue que

$$
2 \sum b_{\lambda}+b_{n}=0
$$

e, como $b_{\lambda} \geq 0$, temos que $b_{\lambda}=0$ para $\lambda=0, \ldots, n$. Mas,

$$
\begin{aligned}
b_{\lambda} & =\sum \operatorname{rank}\left(\partial_{\lambda+1}\right) \\
& =\sum n u l\left(j_{\lambda, i}\right),
\end{aligned}
$$

e daí $n u l\left(j_{\lambda, i}\right)=0$ para $i=1, \ldots, s$ e $\lambda=0, \ldots, n$. Assim, as inclusões

$$
H_{\lambda}\left(M_{a_{1}}\right) \stackrel{j_{\lambda, 1}}{\rightarrow} H_{\lambda}\left(M_{a_{2}}\right) \stackrel{j_{\lambda, 2}}{\rightarrow} \cdots \rightarrow H_{\lambda}\left(M_{a_{s}}\right)=H_{\lambda}(M)
$$

são injetivas. Se $r \in \mathbf{R}$ é tal que $a_{s}<r$ então $M_{r}(\phi)=M$ e a injetividade em homologia é evidente. Suponhamos então que $a_{i-1}<r<a_{i}$ para algum $i$. Então temos as inclusões

$$
H_{\lambda}\left(M_{r}\right) \stackrel{h}{\rightarrow} H_{\lambda}\left(M_{a_{i}}\right) \stackrel{j_{\lambda, i}}{\rightarrow} H_{\lambda}\left(M_{a_{i+1}}\right)
$$

e segue que $h$ é injetora, pois a composta $j_{\lambda, i} \circ h$ o é, já que esta é a restrição a $H_{\lambda}\left(M_{r}(\phi)\right)$ da aplicação injetiva $j_{\lambda, i}$. Portanto, a inclusão $M_{r}(\phi) \hookrightarrow M$ induz monomorfismo em homologia.

Reciprocamente, suponha que para todo $r \in \mathbf{R}$ a inclusão $M_{r}(\phi) \hookrightarrow M$ induz monomorfismo em homologia. Assim, se $s<r$ o diagrama de inclusões 


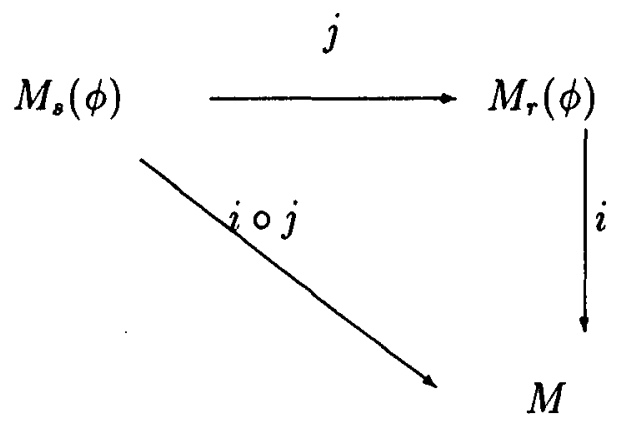

induz o diagrama comutativo em homologia

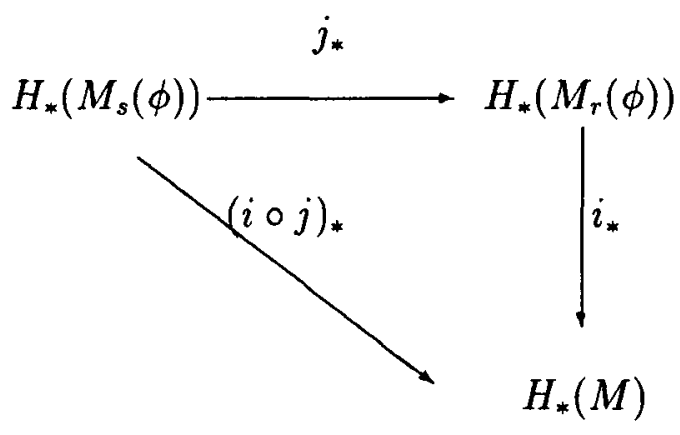

onde as aplicações $i_{*}$ e $(i \circ j)_{*}$ são injetivas. Logo, $j_{*}: H_{\lambda}\left(M_{s}(\phi)\right) \rightarrow H_{\lambda}\left(M_{r}(\phi)\right)$ é injetiva, $\forall s<r$. Então as aplicações $j_{\lambda, i}: H_{\lambda}\left(M_{a_{i-1}}(\phi)\right) \rightarrow H_{\lambda}\left(M_{a_{i}}(\phi)\right)$ são injetivas $\forall \lambda, \forall i$. Logo $b_{\lambda}=0, \forall \lambda$ e da equação 2.4 temos

$$
\sum \mu_{\lambda}(\phi)-\sum \beta_{\lambda}\left(M ; \mathbf{Z}_{2}\right)=0
$$

donde

$$
\begin{aligned}
\mu(\phi) & =\sum \mu_{\lambda}(\phi) \\
& =\sum \beta_{\lambda}\left(M ; \mathbf{Z}_{2}\right) .
\end{aligned}
$$


Corolário 2.1 Se a variedade $M$ satisfaz a condição $3 A$, então $\mu(\phi)=\gamma(M)$ se, somente se, para todo $r \in \mathbf{R}$ a inclusão $M_{r}(\phi) \hookrightarrow M$ induz monomorfismo em homologia.

Lembremos que nosso objetivo é definir algo que seja equivalente à curvatura total absoluta minima. Assim, este lema motiva a definição de justeza dada em termos de injetividade em homologia.

Definição 2.7 Uma aplicação $f: X \rightarrow \mathbf{E}^{m}$ de um espaço topológico compacto $X$ no espaço Euclideano $\mathrm{E}^{m}$ é dita justa se para todo semi-espaço fechado $h$ em $\mathbf{E}^{m}$, o homomorfismo induzido por inclusão

$$
H_{*}\left(f^{-1}(h) ; \mathbf{Z}_{2}\right) \rightarrow H_{*}\left(M ; \mathbf{Z}_{2}\right)
$$

na homologia de $\check{C}$ ech com coeficientes em $\mathbf{Z}_{2}$ é monomorfismo. Um subconjunto de $\mathbf{E}^{m}$ é justo se a inclusão é justa.

Observemos alguns pontos desta definição.

Observação 2.1 A homologia de Čech é usada devido à sua propriedade de continuidade que é necessária para eliminar a hipótese de que o semi-espaço $h$ seja determinado por uma função altura não degenerada. Note que a definição se estende à categoria de imersões suaves de variedades. Para espaços trianguláveis (e então, para variedades), a homologia de Čech coincide com a homologia singular $([10])$.

Observação 2.2 Justeza é propriedade projetiva.

Da definição vemos que para verificar se uma aplicação $f: X \rightarrow \mathbf{E}^{m}$ é justa necessitamos somente do espaço afim $\mathbf{A}^{m}$, e não do espaço métrico $\mathbf{E}^{m}$. O espaço afim $\mathbf{A}^{m}$ pode ser fechado adicionando as classes de retas paralelas como novos pontos, obtendo assim um espaço projetivo real $\mathbf{P}^{m}=\mathbf{A}^{m} \cup \mathbf{P}\left(\mathbf{A}^{\mathbf{m}}\right)$, onde $\mathbf{P}\left(\mathbf{A}^{\mathbf{m}}\right)$ é o conjunto de direções de $\mathbf{A}^{m}$. Seja $p: \mathbf{A}^{m+1} \rightarrow \mathbf{P}^{m}$ a projeção canônica e seja $\phi: \mathbf{A}^{m} \rightarrow \mathbf{P}^{m}$ dada por $\phi\left(x_{1}, \ldots, x_{m}\right)=p\left(x_{1}, \ldots, x_{m}, 1\right)$. Se $\left(x_{1}, \ldots, x_{m}, x_{m+1}\right) \in$ $\mathbf{A}^{m+1} \operatorname{com} x_{m+1} \neq 0$, então

$$
\begin{aligned}
p\left(x_{1}, \ldots, x_{m}, x_{m+1}\right) & =p\left(\frac{x_{1}}{x_{m+1}}, \ldots, \frac{x_{m}}{x_{m+1}}, 1\right) \\
& =\phi\left(\frac{x_{1}}{x_{m+1}}, \ldots, \frac{x_{m}}{x_{m+1}}\right) .
\end{aligned}
$$


Logo, $\mathbf{P}^{m}-\phi\left(\mathbf{A}^{m}\right)=\mathbf{P}^{m-1}$, isto é, $\mathbf{A}^{m}$ é mergulhado por $\phi$ como complemento de um hiperplano $\mathbf{P}^{m-1}$ em $\mathbf{P}^{m}$. Seja $f: X \rightarrow \mathbf{A}^{m}$ uma aplicação justa e $\sigma: \mathbf{P}^{m} \rightarrow \mathbf{P}^{m}$ uma transformação projetiva tal que a imagem $\sigma(f(X))$ está em $\mathbf{A}^{m}$. Então $\sigma f: X \rightarrow \mathbf{A}^{m}$ também é justa. De fato, para todo semi-espaço fechado $h$ em $\mathbf{A}^{m}$ temos

$$
(\sigma f)^{-1} h=f^{-1}\left(\sigma^{-1} h\right)=f^{-1} h^{\prime}
$$

para um semi-espaço $h^{\prime}$ conveniente.

Observação 2.3 Projeçôes ortogonais de aplicaçôes justas são justas.

Seja $f: X \rightarrow \mathbf{E}^{m}$ uma aplicação justa e $\sigma: \mathbf{E}^{m} \rightarrow \mathbf{E}^{k}$ uma projeção ortogonal. Então $\sigma \circ f: X \rightarrow \mathbf{E}^{k}$ é justa. Seja $h$ um semi-espaço fechado de $\mathbf{E}^{k}$ dado por $\ell_{p} \leq r$ para $p \in \mathbf{E}^{k}$. Esta desigualdade quando considerada em $\mathbf{E}^{m}$ dá origem a um subespaço $h^{\prime}$ em $\mathbf{E}^{m}$ que se projeta por $\sigma$ sobre o semi-espaço $h$, isto é, $\sigma^{-1} h=h^{\prime}$. Então,

$$
(\sigma f)^{-1} h=f^{-1}\left(\sigma^{-1} h\right)=f^{-1} h^{\prime},
$$

e a justeza de $\sigma f$ segue da justeza de $f$. Por outro lado, se $f: X \rightarrow \mathbf{E}^{m}$ é justa e $i: \mathbf{E}^{m} \rightarrow \mathbf{E}^{m+j}$ é a inclusão, então $i$ o $f: X \rightarrow \mathbf{E}^{m+j}$ é justa. De fato, se $h$ é um subespaço fechado em $\mathbf{E}^{m+j}$, então $i^{-1} h=h \cap \mathbf{E}^{m}=h^{\prime}$, e portanto,

$$
(i \circ f)^{-1} h=f^{-1}\left(i^{-1} h\right)=f^{-1} h^{\prime} .
$$

Na sequência mostraremos que justeza é equivalente à curvatura total absoluta mínima para imersôes suaves de variedades que satisfazem a condição $3 A$. O ponto central é mostrar que para uma tal imersão, a injetividade em homologia da definição de justeza se verifica para semi-espaços determinados por funções altura degeneradas bem como para as não degeneradas. Para isto faremos uso da continuidade da homologia de Cech e do lema abaixo. Fixemos as notações:

$$
\begin{aligned}
M_{r}(p) & =\left\{x \in M ; \ell_{p}(x) \leq r\right\} \\
M_{r}^{-}(p) & =\left\{x \in M ; \ell_{p}(x)<r\right\} .
\end{aligned}
$$

Lema 2.3 Seja $f: M^{n} \rightarrow \mathbf{E}^{m}$ uma imersão da variedade compacta $M$. Suponha que $U$ é um aberto de $M$ que contém $M_{r}(p)$ para algum $p \in S^{m-1}, r \in \mathbf{R}$. Então existe uma função altura não degenerada $\ell_{q}$ e $s \in \mathbf{R}$ tal que

$$
M_{r}(p) \subset M_{s}^{-}(q) \subset M_{s}(q) \subset U .
$$




\section{Demonstração:}

Como $M_{r}(p)$ é compacto e $U$ é aberto, existe $\epsilon>0$ tal que $M_{r+\epsilon}(p) \subset U$. Sejam $k$ o valor máximo que qualquer função altura pode assumir em $M$ e $p^{\prime} \epsilon$ $S^{m-1}$ um vetor ortogonal a $p$. Seja $q=\cos (\alpha) p+\operatorname{sen}(\alpha) p^{\prime}, \operatorname{com} \cos (\alpha) \neq 0$. Então,

$$
p=\sec (\alpha) q-\tan (\alpha) p^{\prime}
$$

e dado $x \in M$, temos

$$
\begin{aligned}
\left|\ell_{p}(x)-\ell_{q}(x)\right| & =|<p-q, f(x)>| \\
& =\left|<(\sec (\alpha)-1) q-\tan (\alpha) p^{\prime}, f(x)>\right| \\
& =\left|(\sec (\alpha)-1) \ell_{q}(x)-\tan (\alpha) \ell_{p^{\prime}}(x)\right| \\
& =\leq|\sec (\alpha)-1|\left|\ell_{q}(x)\right|+|\tan (\alpha)|\left|\ell_{p^{\prime}}(x)\right| \\
& =\leq(|\sec (\alpha)-1|+|\tan (\alpha)|) k
\end{aligned}
$$

Escolha $\alpha$ pequeno tal que

$$
|\sec (\alpha)-1|<\frac{\epsilon}{4 k}, e|\tan (\alpha)|<\frac{\epsilon}{4 k} .
$$

Então,

$$
\left|\ell_{p}(x)-\ell_{q}(x)\right|<\frac{\epsilon}{2}, \forall x \in M
$$

e portanto,

$$
M_{r}(p) \subset M_{r+\epsilon / 2}^{-}(q) \subset M_{r+\epsilon / 2}(q) \subset M_{r+\epsilon}(p) \subset U,
$$

para todo $q \in S^{m-1}$ na vizinhança de $p$ determinada por $\alpha$, e portanto para algum $q \operatorname{com} \ell_{q}$ não degenerada.

Estamos agora em condições de provar o teorema principal deste capítulo que dá uma importante caracterização de justeza.

Teorema 2.6 (Kuiper, [13]) Uma imersão $f: M \rightarrow \mathbf{E}^{m}$ da variedade compacta $M$ é justa se, e somente se, $f$ tem curvatura total absoluta minima e $M$ satisfaz a condiçâo $\gamma(M)=\sum \beta_{i}\left(M ; \mathbf{Z}_{2}\right)=\beta\left(M ; \mathbf{Z}_{2}\right)$.

\section{Demonstração:} fismo

Se $f$ é justa e $\ell_{p}$ é qualquer função altura não degenerada então o homomor-

$$
H_{*}\left(M_{r}(p)\right) \rightarrow H_{*}(M)
$$


induzido por inclusão, é injetivo. Do lema 2.2 segue que

$$
\mu(p)=\beta\left(M ; \mathbf{Z}_{2}\right)
$$

Então, $\gamma(M) \leq \tau(M ; f)=\beta\left(M ; \mathbf{Z}_{2}\right)$. Por outro lado, pelo corolário 1.2 temos $\gamma(M) \geq \beta\left(M ; \mathbf{Z}_{2}\right)$. Logo, $\gamma(M)=\beta\left(M ; \mathbf{Z}_{2}\right)$ e $f$ tem curvatura total absoluta mínima.

Reciprocamente, suponha que $f$ tem curvatura total absoluta mínima e que $\gamma(M)=\beta\left(M ; \mathbf{Z}_{2}\right)$. Então, pelo lema 2.2 o homomorfismo

$$
H_{*}\left(f^{-1} h\right) \rightarrow H_{*}(M)
$$

é injetivo para cada semi-espaço $h$ determinado por uma função altura não degenerada. Suponhamos então que $f^{-1} h=M_{r}(p)$ para uma função altura $\ell_{p}$ degenerada e $r \in \mathbf{R}$. Devemos mostrar que o homomorfismo

$$
H_{*}\left(M_{r}(p)\right) \rightarrow H_{*}(M)
$$

é ainda injetivo. Para tanto, faremos uso da continuidade da homologia de Čech e do lema 2.3. Com o auxílio do lema 2.3 produziremos uma sequência encaixada de semi-espaços $h_{i}, i=1,2, \ldots$, satisfazendo

$$
f^{-1}\left(h_{i}\right) \supset f^{-1}\left(h_{i+1}\right) \supset \cdots \supset \cap_{j=1}^{\infty} f^{-1}\left(h_{j}\right)=M_{r}(p), i=1,2, \ldots,
$$

tal que o homomorfismo em $\mathbf{Z}_{2}$-homologia de Čech

$$
H_{*}\left(f^{-1}\left(h_{i}\right)\right) \rightarrow H_{*}(M), i=1,2, \ldots,
$$

seja injetivo. Se as equações 2.5 e 2.6 estiverem satisfeitas, então

$$
H_{*}\left(f^{-1}\left(h_{i}\right)\right) \rightarrow H_{*}\left(f^{-1}\left(h_{j}\right)\right)
$$

é injetivo para $i>j$. Pela continuidade da homologia de Čech, temos

$$
H_{*}\left(M_{r}(p)\right)=H_{*}\left(\stackrel{\leftarrow}{\lim } f^{-1}\left(h_{i}\right)\right)=\stackrel{\leftarrow}{\lim } H_{*}\left(f^{-1}\left(h_{i}\right)\right) .
$$

Da equação 2.7 e do teorema 3.4 de Eilenberg-Steenrood ([10]), segue que o homomorfismo

$$
H_{*}\left(M_{r}(p)\right) \rightarrow H_{*}\left(f^{-1}\left(h_{i}\right)\right)
$$

é injetivo para todo $i$. Então também será injetivo o homomorfismo

$$
H_{*}\left(M_{r}(p)\right) \rightarrow H_{*}(M)
$$


Para concluir a demonstração basta construir tal sequência. Produziremos a sequência $\left\{h_{i}\right\}$ indutivamente usando o lema 2.3. Para $i=1$ seja $U_{1}=M_{r+1}^{-}(p)$. Pelo lema 2.3 existem $\ell_{q_{1}}$ não degenerada e $s_{1} \in \mathbf{R}$ tal que

$$
M_{r}(p) \subset M_{s_{1}}^{-}(q) \subset M_{s_{1}}(q) \subset U_{1} .
$$

Tome $h_{1}=\left\{x \in \mathbf{E}^{m} ; \ell_{q_{1}}(x) \leq s_{1}\right\}$. Suponha que tenhamos construido $h_{1} \supset h_{2} \supset$ .. つ $h_{i-1}$. Seja $U=M_{s^{\prime}}^{-}\left(q^{\prime}\right)$, onde $s^{\prime}$ e $q^{\prime}$ são obtidos previamente do lema 2.3 satisfazendo

$$
M_{r}(p) \subset M_{s^{\prime}}^{-}\left(q^{\prime}\right) \subset M_{s^{\prime}}\left(q^{\prime}\right) \subset M_{r+1 / i}(p) .
$$

Novamente pelo lema 2.3 existe $\ell_{q}$ não degenerada e $s \in \mathbf{R}$ tal que

$$
M_{r}(p) \subset M_{s}^{-}(q) \subset M_{s}(q) \subset M_{s^{\prime}}\left(q^{\prime}\right) .
$$

Então $h_{i}$ é o semi-espaço $\ell_{q} \leq s$ obtido no i-ésimo passo da indução e a sequência $h_{i}$ é encaixada. Note que 2.6 se verifica pois $\ell_{q}$ é não degenerada. Como, por construção, $f^{-1}\left(h_{i+1}\right) \subset M_{r+1 / i}(p)$ segue que $\cap_{j=1}^{\infty} f^{-1}\left(h_{j}\right)=M_{r}(p)$, e o teorema está provado.

Observação 2.4 Existem exemplos de variedades com curvatura total absoluta mínima que não sâo justas. Estes exemplos foram construídos por Kuiper e Meeks em [17].

Definição 2.8 Seja $\phi: M \rightarrow \mathbf{R}$ uma função de Morse. Dizemos que $\phi$ é polar se ela tem exatamente um máximo e um mínimo local.

Observação 2.5 Para uma variedade compacta e conexa $M$, Morse (ver [6]), mostrou que se uma funçâo nâo degenerada $\phi$ tem mais que um máximo ou mínimo local, é possivel construir uma nova função não degenerada $\varphi$ que é polar cujo número de pontos críticos é menor que o número de pontos críticos de $\phi$. Segue que se $\mu(\phi)=\gamma(M)$ então $\phi$ é polar.

Proposição 2.1 Seja $M$ uma superfície conexa e compacta 2-dimensional. Então, (a) $\gamma(M)=\beta\left(M ; \mathbf{Z}_{2}\right)=4-\chi(M)$, onde $\chi(M)$ é a caracteristica de Euler de $M$; (b) Para uma função não degenerada $\phi$ em $M, \mu(\phi)=\gamma(M)$ se, e somente se, $\phi$ é polar.

\section{Demonstração:}


Provaremos (a) e (b) simultaneamente. Pela observação 2.5 acima se $\mu(\phi)=$ $\gamma(M)$ para alguma $\phi$ não degenerada então $\phi$ é polar. Por outro lado, suponha que $\phi: M^{2} \rightarrow \mathbf{R}$ é polar. Então, $\mu_{0}(\phi)=1=\beta_{0}\left(M ; \mathbf{Z}_{2}\right)$ e, como estamos trabalhando com coeficientes em $\mathbf{Z}_{2}$, também $\mu_{2}(\phi)=1=\beta_{2}\left(M ; \mathbf{Z}_{2}\right)$. Estas informações junto com a relação de Morse (teorema 1.5)

$$
\sum_{k=0}^{2}(-1)^{k} \mu_{k}(\phi)=\sum_{k=0}^{2}(-1)^{k} \beta_{k}\left(M ; \mathbf{Z}_{2}\right)=\chi(M),
$$

implicam que $\mu_{1}(\phi)=\beta_{1}\left(M ; \mathbf{Z}_{2}\right)=2-\chi(M)$. Assim, se $\phi$ é qualquer função polar não degenerada em $M$,então $\mu(\phi)=\beta\left(M ; \mathbf{Z}_{2}\right)$. Então qualquer função não degenerada em $M$ tem pelo menos $\beta\left(M ; \mathbf{Z}_{2}\right)$ pontos críticos. Como $\gamma(M)=$ $\inf \{\mu(\phi) ; \phi \dot{e}$ de Morse $\}$ segue que $\gamma(M)=\beta\left(M ; Z_{2}\right)=4-\chi(M)$, e que se $\phi \dot{e}$ polar então $\mu(\phi)=\gamma(M)$.

Corolário 2.2 Seja $M$ uma superfície compacta e conexa de dimensão 2 e $f: M \rightarrow \mathbf{E}^{m}$ uma imersão. Então são equivalentes as afirmações:
(a) $f$ é justa;
(b) $f$ tem curvatura total absoluta mínima;
(c) toda função altura não degenerada é polar. 


\section{Capítulo 3}

\section{A Propriedade dos Dois Pedaços}

Introduziremos neste capítulo a propriedade dos dois pedaços (PDP) de Banchoff que será bastante útil no estudo das imersões justas em variedades de dimensão 2. A importância desta propriedade está no fato de que em dimensão 2, ela coincide com justeza. Embora, em geral, seja mais fraca que justeza, o significado geométrico da PDP é bem mais simples.

Definição 3.1 Uma aplicação $f: X \rightarrow \mathbf{E}^{m}$ onde $X$ é um espaço topológico conexo tem a propriedade dos dois pedaços (PDP) se $f^{-1} h$ é conexo para todo semi-espaço fechado $h$ em $\mathbf{E}^{m}$.

A interpretação geométrica da PDP é bastante simples: para um espaço $X$ mergulhado em $\mathbf{E}^{m}$, ter a PDP implica que todo hiperplano $\Pi$ corta $X$ em no máximo duas partes.

Seja $f: X \rightarrow \mathbf{E}^{m}$ uma aplicação justa. O número $\beta_{0}\left(f^{-1} h ; \mathbf{Z}_{2}\right)$ conta o número de componentes conexas de $f^{-1} h$. Como $f$ é justa, o homomorfismo

$$
H_{0}\left(f^{-1} h ; \mathbf{Z}_{2}\right) \rightarrow H_{0}\left(X ; \mathbf{Z}_{2}\right)
$$

é injetivo. Mas, como $X$ é conexo, $\beta_{0}\left(X ; Z_{2}\right)=1$. Logo, pela injetividade segue que $\beta_{0}\left(f^{-1} h ; Z_{2}\right)=1$ para qualquer semi-espaço fechado $h$, e portanto $f$ tem a propriedade dos dois pedaços. Provamos assim, o

Teorema 3.1 Seja $f: X \rightarrow \mathbf{E}^{m}$ uma aplicação contínua do espaço topológico $X$ em $\mathbf{E}^{m}$. Se $f$ é justa, então $f$ tem a PDP. 


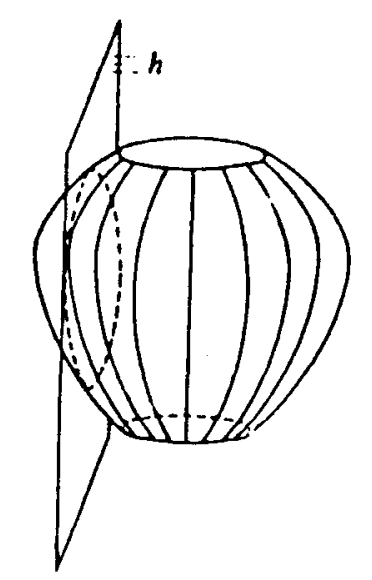

Figura 3.1: Anel PDP não justo

A recíproca é falsa. Vejamos um exemplo.

Exemplo 1 Seja $X$ o anel dado por

$$
x^{2}+y^{2}+z^{2}=1,-1 / \sqrt{2} \leq z \leq 1 / \sqrt{2}
$$

Claramente $X$ tem a PDP. Seja $h$ o semi-espaço $x \geq-3 / 4$ e $X \cap h=\{(x, y, z) \in$ $X ; x \geq-3 / 4\}$. O hiperplano $h$ não intercepta os círculos da fronteira de $X$, isto é, os círculos

$$
\left\{\begin{array}{l}
x^{2}+y^{2}=1 \\
z= \pm 1 / \sqrt{2}
\end{array}\right.
$$

e portanto, $X \cap h$ é um anel com um buraco. Assim, $H_{1}\left(X \cap h ; \mathbf{Z}_{2}\right)$ tem dois geradores, isto é, $H_{1}\left(X \cap h ; \mathbf{Z}_{2}\right)=\mathbf{Z}_{2} \oplus \mathbf{Z}_{2}$ enquanto que $H_{1}\left(X ; \mathbf{Z}_{2}\right)=\mathbf{Z}_{2}$.

O teorema 3.1 e o exemplo acima mostram que a PDP é mais fraca que justeza. No que segue relacionaremos os principais resultados sobre a PDP.

Proposição 3.1 Seja $f: X \rightarrow \mathbf{E}^{m}$ uma aplicação contínua de um espaço compacto. Então $f$ tem a propriedade dos dois pedaços se, e somente se, $f^{-1}(h)$ é conexo para todo semi-espaço aberto $h$ em $\mathbf{E}^{m}$.

Esta proposição diz que podemos substituir semi-espaço fechado por semiespaço aberto na definição 3.1. A demonstração pode ser encontrada em [6]. 
Teorema 3.2 Seja $f: M \rightarrow \mathbf{E}^{m}$ uma imersão definida na variedade compacta e conexa $M$. Então $f$ tem a propriedade dos dois pedaços se, e somente se, toda função altura não degenerada é polar.

Este teorema e o corolário 2.2 juntos dão o seguinte

Corolário 3.1 Uma imersão $f: M \rightarrow \mathbf{E}^{m}$ com a propriedade dos dois pedaços, onde $M$ é uma variedade de dimensão 2 compacta e conexa, é justa.

A demonstração do teorema 3.2 seguirá dos seguintes resultados.

Proposição 3.2 Seja $f: M \rightarrow \mathbf{E}^{m}$ uma imersão de uma variedade compacta e conexa. Uma função altura não degenerada $\ell_{p}$ é polar se, e somente se, $f^{-1} h$ é conexo para todo semi-espaço fechado $h$ determinado por $\ell_{p}$.

\section{Demonstração:}

Suponha que para alguma $\ell_{p}$ não degenerada o semi-espaço

$$
h=\left\{y \in \mathbf{E}^{m} ; \ell_{p}(y) \geq r\right\}
$$

seja tal que $f^{-1} h$ tenha pelo menos duas componentes conexas $V_{1}$ e $V_{2}$. Então $\ell_{p}$ tem pelo menos dois máximos locais, sendo um em $V_{1}$ e outro em $V_{2}$, e portanto não é polar.

Por outro lado, suponha que uma $\ell_{p}$ não degenerada tenha dois máximos locais $x_{1}$ e $x_{2}$ (se $\ell_{p}$ tem dois mínimos, $\ell_{-p}$ tem dois máximos) em alturas $r_{1}$ e $r_{2}$ com $r_{1} \leq r_{2}$. Pelo lema de Morse 1.1 existe uma vizinhança $U$ de $x_{1}$ não contendo $x_{2}$ tal que para $\epsilon>0$ suficientemente pequeno o conjunto fechado

$$
V=\left\{x \in \bar{U} ; \ell_{p}(x) \geq r_{1}-\epsilon\right\}
$$

está contido no interior de $U$. Seja $h$ o semi-espaço fechado determinado pela equação $\ell_{p} \geq r_{1}-\epsilon$. Então $V$ é uma componente de $f^{-1} h$, mas $x_{2} \in f^{-1} h-V$. Portanto, $f^{-1} h$ não é conexo.

Para completarmos a prova do teorema 3.2 , devemos mostrar que se toda função altura não degenerada é polar então $f^{-1} h$ é conexo para todo semi-espaço $h$ determinado por funções altura degeneradas. Isto pode ser feito usando a homologia de Čech, como na prova do teorema 2.6, mas o lema a seguir possibilita uma prova bem mais elementar. 
Lema 3.1 Seja $f: M \rightarrow \mathbf{E}^{m}$ uma imersão. Suponha que $W \subset U \subset \bar{U} \subset M$, onde $U$ é um aberto tal que para algum $p \in S^{m-1}$ tenha-se $\ell_{p}(x)=\alpha$ para $x \in W$ e $\ell_{p}(x)<\alpha$ para $x \in \bar{U}-W$. Então existe uma vizinhança $N$ de $p$ em $S^{m-1}$ tal que se $q \in N$ então $\ell_{q}$ assume seu máximo em $\bar{U}$ no interior de $U$.

\section{Demonstração:}

Seja $\alpha-3 \epsilon, \epsilon>0$, o valor máximo de $\ell_{p}$ em $\partial U$. Como na prova do lema 2.3 existe uma vizinhança $N$ de $p$ em $S^{m-1}$ tal que para todo $q \in N$,

$$
\left|\ell_{p}(x)-\ell_{q}(x)\right|<\epsilon, \forall x \in \bar{U}
$$

Então, para cada $x \in W$, temos $\ell_{q}(x) \geq \alpha-\epsilon$, enquanto que para $x \in \partial U$, temos $\ell_{q}(x)<\alpha-2 \epsilon$. Portanto, $\ell_{q}$ assume seu máximo em $\bar{U}$ no interior de $U$. Note que, se $\ell_{q}$ é não degenerada, isto significa que $\ell_{q}$ tem um máximo local em um ponto de $U$.

\section{Demonstração do teorema 3.2:}

Se $f$ tem a PDP, então pela proposição 3.2 , toda $\ell_{q}$ não degenerada é polar. Por outro lado, suponha que $f$ não tem a PDP. Então existe um semiespaço fechado $h$ tal que $f^{-1} h$ tem pelo menos duas componentes conexas $V_{1}$ e $V_{2}$. Suponha que $h$ é dado por $\ell_{p} \geq r$. Como $V_{1}$ e $V_{2}$ são compactos, existem abertos $U_{1}$ e $U_{2}$ com $V_{i} \subset U_{i}$. A função $\ell_{p}$ assume seus valores máximos em $U_{i}$ em pontos de $V_{i}$. Aplicando o lema 3.1 a $U_{1}$ e $U_{2}$, concluimos que existe uma $\ell_{q}$ não degenerada com $q$ perto de $p$, que tem um máximo local em $U_{1}$ e outro em $U_{2}$ e, portanto não é polar.

Para uma melhor caracterização de imersões com a PDP, formalizaremos esta noção em termos de planos suportes.

Definição 3.2 Seja $f: M \rightarrow \mathbf{E}^{m}$ uma imersão de uma variedade compacta. Um hiperplano $\Pi$ em $\mathbf{E}^{m}$ passando pelo ponto $f(x)$ é dito um plano suporte local de $f(M)$ em $x$ se existe uma vizinhança $U$ de $x$ tal que $f(U)$ está inteiramente contido em um semi-espaço fechado determinado por $\Pi$ e $f(\partial U)$ está no interior deste semi-espaço.

Teorema 3.3 Se $f: M \rightarrow \mathbf{E}^{m}$ é uma imersão com a PDP e $M$ é compacta, então todo plano suporte local de $f(M)$ é um plano suporte (global) de $f(M)$.

\section{Demonstração:}


Seja $\Pi$ um plano suporte local em $x \in M$ dado pela equação $\ell_{p}=r$. Então existe vizinhança $U$ de $x$ em $M$ tal que $f(U) \subset\left\{\ell_{p} \leq r\right\}$ e $f(\partial U) \subset\left\{\ell_{p}<r\right\}$. Suponha que $\Pi$ não é plano suporte global de $f(M)$. Então existe $y \in M$ tal que $f(y) \in\left\{\ell_{p}>r\right\}$, isto é, $\ell_{p}(y)>r$. Se $h$ é o semi-espaço determinado por $\ell_{p} \geq r$, então $f^{-1} h$ tem pelo menos duas componentes conexas: uma contida em $U$ e que contém o ponto $x$ e a outra em $M-\bar{U}$ contendo $y$, contrariando a PDP.

A recíproca deste teorema é falsa. De fato, se $f: S^{1} \rightarrow \mathbf{E}^{2}$ é uma imersão que dá duas voltas ao redor do círculo unitário em velocidade constante, então todo plano suporte local é também global, mas $f$ não tem a PDP. Entretanto a recíproca é verdadeira se $f$ é um mergulho.

Teorema 3.4 Seja $f: M \rightarrow \mathbf{E}^{m}$ um mergulho diferenciável da variedade compacta $M$. Então as afirmações são equivalentes:

(a) f tem a PDP;

(b) todo plano suporte local de $f(M)$ é plano suporte global;

(c) todo extremo local de uma função altura não degenerada é um extremo absoluto.

\section{Demonstração:}

(a) $\Rightarrow$ (b) pelo teorema 3.3.

(b) $\Rightarrow(\mathrm{c})$

Se uma $\ell_{p}$ não degenerada tem um extremo local em $x$ que não é um extremo absoluto, então pelo Lema de Morse 1.1, o hiperplano $\ell_{p}=r$, onde $r=\ell_{p}(x)$ é um plano suporte local, mas no entanto não é plano suporte global.

$$
\text { (c) } \Rightarrow(\mathrm{a})
$$

Suponha que $f$ não tem a PDP. Então, pelo teorema 3.2 existe uma $\ell_{p}$ não degenerada com pelo menos dois máximos $x_{1}, x_{2}$. Se estes máximos estão em alturas diferentes, a prova está terminada. Suponhamos então que $\ell_{p}\left(x_{1}\right)=\ell_{p}\left(x_{2}\right)$ é o valor máximo absoluto de $\ell_{p}$ em $M$. Através de uma translação podemos assumir que $f\left(x_{1}\right)=0$ e então $\ell_{p}\left(x_{1}\right)=\ell_{p}\left(x_{2}\right)=0$ é o máximo de $\ell_{p}$ em $M$. Observe que, como $f$ é mergulho $f\left(x_{2}\right) \neq 0$. Considere então o vetor unitário $q=\frac{f\left(x_{2}\right)}{\epsilon}$, onde $\epsilon=\left|f\left(x_{2}\right)\right|$. Sejam $U_{1}=f^{-1}\left(B\left(f\left(x_{1}\right), \epsilon / 3\right)\right)$ e $U_{2}=f^{-1}\left(B\left(f\left(x_{2}\right), \epsilon / 3\right)\right)$. Então, o máximo de $\ell_{q}$ em $U_{1}$ é $\epsilon / 3$ e o mínimo de $\ell_{q}$ em $U_{2}$ é $2 \epsilon / 3$. Sejam $N_{1}$ e $N_{2}$ vizinhanças de $p$ em $S^{m-1}$ obtidas aplicando-se o lema 3.1 a $U_{1}$ e $U_{2}$, respectivamente. Seja $p^{\prime}=\cos (\alpha) p+\operatorname{sen}(\alpha) q$ um ponto em $N_{1} \cap N_{2}$ para algum $\alpha$ pequeno, $0<\alpha<\pi / 2$. Novamente, pelo lema $3.1, \ell_{p^{\prime}}$ tem um valor máximo local em $U_{1}$ e outro em $U_{2}$. Se $y \in U_{1}$, temos

$$
\ell_{p^{\prime}}(y)=\cos (\alpha) \ell_{p}(y)+\operatorname{sen}(\alpha) \ell_{q}(y) \leq(\epsilon / 3) \operatorname{sen}(\alpha)
$$


e por outro lado,

$$
\ell_{p^{\prime}}\left(x_{2}\right)=\cos (\alpha) \ell_{p}\left(x_{2}\right)+\operatorname{sen}(\alpha) \ell_{q}\left(x_{2}\right)=\operatorname{sen}(\alpha)<\frac{f\left(x_{2}\right)}{\epsilon}, f\left(x_{2}\right)>=\epsilon \operatorname{sen}(\alpha) .
$$

Então, os máximos de $\ell_{p^{\prime}}$ em $U_{1}$ e $U_{2}$ estão em alturas diferentes. Se $\ell_{p^{\prime}}$ é degenerada, pela parte (b) do corolario 1.4, existe uma função altura não degenerada $\ell_{v}, v$ próximo de $p^{\prime}$, que tem máximos locais em $U_{1}$ e $U_{2}$ em alturas diferentes.

Definição 3.3 Uma aplicação $f: X \rightarrow \mathbf{E}^{m}$ onde $X$ é um espaço topológico é dita substancial se a imagem $f(X)$ não está contida em nenhum hiperplano de $\mathbf{E}^{m}$.

O principal resultado sobre imersões PDP substanciais é o seguinte teorema de Kuiper que estabelece um limite superior para a codimensão.

Teorema 3.5 (Kuiper) Seja $f: M \rightarrow \mathbf{E}^{m}$ uma imersão diferenciável substancial da variedade compacta n-dimensional $M$. Se $f$ tem a PDP, então $m \leq \frac{1}{2} n(n+3)$.

\section{Demonstração:}

Seja $\ell_{p}$ uma função altura linear não degenerada em $M$ com máximo absoluto em $x \in M$. Após uma translação, podemos assumir que $f(x)$ é a origem de $\mathbf{E}^{m}, \mathbf{e}$ então $\ell_{p}(x)=0$. Pelo teorema 1.9 sabemos que $p$ é normal a $f(M)$ em $f(x)$ e que a Hessiana de $\ell_{p}$ em $x, H(u, v)=\left\langle A_{p} u, v>\right.$, é negativa definida. Seja $\left(T_{x} M\right)^{\perp}$ o espaço normal a $f(M)$ em $f(x)$ e seja $V$ o espaço vetorial das formas bilineares simétricas em $T_{x} M$. Defina a aplicação linear $\phi:\left(T_{x} M\right)^{\perp} \rightarrow V$ por

$$
\phi(q)=A_{q}
$$

isto é,

$$
\phi(q)(u, v)=<A_{q} u, v>, \forall u, v \in T_{x} M .
$$

A dimensão de $\left(T_{x} M\right)^{\perp}$ é $m-n$, enquanto $V$ tem dimensão $\frac{1}{2} n(n+1)$. Assim, se $m-n>\frac{1}{2} n(n+1)$, ou seja, $m>\frac{1}{2} n(n+3)$, então o núcleo de $\phi$ é não trivial. Completaremos a prova mostrando que a PDP implica a injetividade de $\phi$. Suponha que exista $q \neq 0$ em $\left(T_{x} M\right)^{\perp} \operatorname{com} A_{q}=0$. Seja $z(t)=p+t q$. Então $z(t) \in\left(T_{x} M\right)^{\perp}, \forall t, \mathbf{e}$

$$
A_{z(t)}=A_{p}+t A_{q}=A_{p}, \forall t
$$


Logo, $\ell_{x(t)}$ tem um máximo não degenerado em $x, \forall t$. Por outro lado, como $f$ é substancial, existe um ponto $y \in M$ tal que $\ell_{q}(y) \neq 0$. Então,

$$
\ell_{z(t)}(y)=\ell_{p}(y)+t \ell_{q}(y)
$$

e para uma escolha conveniente de $t$, temos $\ell_{z(t)}(y)>0 . \operatorname{Mas} \ell_{z(t)}(x)=t \ell_{q}(x)=0$. Logo, para este valor de $t x$ é máximo local mas não é máximo global de $\ell_{z(t)}$, e então pelo teorema $3.3, f$ não tem a PDP.

Existem exemplos de imersões substanciais e justas de variedades de dimensão $n$ em $\mathrm{E}^{m}$, com $m=\frac{1}{2} n(n+3)$, mostrando que este limite é o melhor possivel (cap. 9 de [6]). O método da prova do teorema anterior dá resultados interessantes se assumirmos $f$ justa.

Teorema 3.6 (Chern-Lashof) Se $f: S^{n} \rightarrow \mathbf{E}^{m}$ é uma imersão diferenciável justa e substancial, então $m=n+1$.

\section{Demonstração:}

Seja $\phi:\left(T_{x} S^{n}\right)^{\perp} \rightarrow V$ como no teorema 3.5. Como antes a PDP (e portanto justeza) implica a injetividade de $\phi$. Sejam $p$ e $q$ linearmente independentes em $\left(T_{x} S^{n}\right)^{\perp}$. Então $A_{p}$ e $A_{q}$ não são múltiplos escalares um do outro, pois caso contrário, pela injetividade de $\phi$ teríamos $p$ e $q$ linearmente dependentes. Podemos supor, que $\ell_{p}$ e $\ell_{q}$ são não degeneradas e que o índice de ambas no ponto crítico $x$ é igual a zero, isto é, as hessianas $H_{x}(p)$ e $H_{x}(q)$ são definidas positivas. As.formas quadráticas $A_{p}$ e $A_{q}$ são simultaneamente diagonalizáveis. De fato, inicialmente reduzimos $A_{q}$ à forma diagonal $A_{q}=\left(b_{i}\right)$. Em seguida, com uma mudança de escala podemos assumir que $b_{i}=1$. Observe que as mudanças efetuadas não afetam o índice das formas quadráticas. Finalmente, como $A_{q}$ é invariante, efetuamos um movimento rígido para diagonalizar a forma quadrática $A_{p}$, digamos $A_{p}=\left(a_{i}\right)$. Assim, o operador $A_{z(t)}$ tem a forma matricial abaixo

$$
\left(\begin{array}{cccr}
a_{1}+t & 0 & \cdots & 0 \\
0 & a_{2}+t & \cdots & 0 \\
0 & 0 & \ddots & 0 \\
0 & 0 & \cdots & a_{n}+t
\end{array}\right)
$$

Devem existir indices $i \neq j$ com $a_{i} \neq a_{j}$, pois se fosse $a_{1}=a_{2}=\cdots=a_{n}=a$, tomando $t=-a$, teríamos $A_{p}+t A_{q}=0$. Digamos então que seja $a_{1}<a_{2}$. Então é possível escolher um $t$ conveniente de tal forma que $a_{i}+t \neq 0$ para todo $i$ com $a_{1}+t<0$ e $a_{2}+t>0$. Logo, para este valor de $t$, o operador $A_{z(t)}$ é não 
degenerado e tem índice $k$ diferente de 0 e $n$. Então $\ell_{z(t)}$ tem um ponto crítico não degenerado de índice $k$ em $x$, e pelo corolário 1.4 existe uma função de Morse $\ell_{p^{\prime}}$ em $S^{n}$ que tem um ponto crítico não degenerado de índice $k$, o que não é permitido pela justeza. Concluímos então que $\left(T_{x} S^{n}\right)^{\perp}$ deve ter dimensão 1 , isto é, $m=n+1$.

Mais adiante veremos que na verdade $f$ mergulha $S^{n}$ sobre uma hipersuperfície convexa em $\mathbf{E}^{n+1}$. Para isto, é necessário assumir somente que $f$ é uma imersão justa substancial topológica.

Banchoff ([2]) mostrou que para todo $n \geq 3$ e para qualquer $m>n$, existem mergulhos substanciais poliedrais de $S^{n}$ em $\mathbf{E}^{m}$ que tem a PDP. Então, a diferenciabilidade no teorema 3.5 é essencial. Mesmo se assumirmos $f$ justa, esta hipótese ainda é necessária.

Apresentaremos a seguir um exemplo de um mergulho PDP de $S^{3}$ em $\mathbf{E}^{4}$ que não é justo. Precisamos da seguinte definição.

Definição 3.4 Uma aplicação $f: X \rightarrow \mathbf{E}^{m}$, onde $X$ é um espaço topológico compacto e conexo, é dita k-justa se para todo semi-espaço fechado $h$ em $\mathbf{E}^{m} e$ para todo inteiro $i \leq k$ o homomorfismo induzido por inclusão

$$
H_{i}\left(f^{-1} h\right) \rightarrow H_{i}(x)
$$

em $\mathrm{Z}_{2}$-homologia é injetivo.

Desta forma 0 -justeza é a propriedade dos dois pedaços e $n$-justeza é justeza. Se $f: M \rightarrow \mathbf{E}^{m}$ é uma imersão diferenciável de uma variedade compacta, então $f$ é k-justa se, e somente se, toda função altura não degenerada $\ell_{p}$ tem $\beta_{i}\left(M ; \mathbf{Z}_{2}\right)$ pontos críticos de índice $i, 0 \leq i \leq k$.

Exemplo $2 S^{3}$ mergulhada em $\mathbf{E}^{4}$ com a PDP mas não justa.

Construção: Começamos com um toro de revolução em $\mathbf{E}^{3}$ com o eixo $y$ como eixo de rotação. Tomamos então sua metade inferior e cobrimos seus fins abertos diferenciavelmente com superfícies convexas. Estas operações preservam a simetria em relação aos planos $x z$ e $y z$, e dão uma superfície $S$ do tipo

$$
\varphi\left(x^{2}, y^{2}, z\right)=0 \text {. }
$$

Um exemplo algébrico é dado por

$$
\left(\frac{x}{4}\right)^{2}+y^{2}+(z-x)^{2}=1
$$


Para obter o mergulho $M$ de $S^{3}$ em $\mathbf{E}^{4}$ desejado rotacionamos a superfície $S$ dada em 3.1 ou 3.2 , ao redor do plano $y z$, isto é, trocamos $x^{2}$ por $x^{2}+w^{2}$, obtendo

$$
\frac{x^{2}+w^{2}}{16}+y^{2}+\left(z-\left(x^{2}+w^{2}\right)\right)^{2}=1 .
$$

Existem funções altura com pontos críticos não degenerados de índice 1 ou 2 em $M$. De fato, parametrize $M$ por

$$
f:(x, z, w) \rightarrow\left(x, \sqrt{1-\frac{x^{2}+w^{2}}{16}-\left(z-\left(x^{2}+w^{2}\right)\right)^{2}}, z, w\right) .
$$

Como $M$ é dada pelo gráfico de

$$
y=\sqrt{1-\frac{x^{2}+w^{2}}{16}-\left(z-\left(x^{2}+w^{2}\right)\right)^{2}}
$$

a direção normal em um ponto $Q$ é dada por

$$
v(Q)=\left(y_{x},-1, y_{z}, y_{w}\right) .
$$

Logo, a função altura será dada por

$$
\ell_{v(Q)}(x, z, w)=x y_{x}(Q)-y+z y_{z}(Q)+w y_{w}(Q),
$$

e sua Hessiana em $Q$ é dada por

$$
H_{Q}\left(\ell_{v(Q)}\right)=-H_{Q}(y)
$$

As derivadas mistas de $y$ e então as de $\ell_{v(Q)}$ se anulam sobre o círculo $z^{2}+y^{2}=1$. Além disso temos também $y_{z z}<0$ e $y_{x x}, y_{w w}>0$ para $z^{2}+y^{2}=1, \quad z>0$. Portanto, existem funções altura com pontos críticos não degenerados de índice 1 ou 2 em $M$. Logo, $M$ não é um mergulho justo de $S^{3}$. Para mostrar que $M$ tem a PDP mostraremos que todo extremo local de funções altura estão na fronteira do fecho convexo de $M$ e serão então extremos globais. Seja $\ell_{p}: \mathbf{E}^{4} \rightarrow \mathbf{R}$ uma função altura não degenerada. Pela simetria de $M$ obtemos os mesmos valores críticos e índices para $\ell_{p}$ e para uma $\ell_{p^{\prime}}$ obtida pela rotação das variáveis $x \mathrm{e} w$. Então, podemos assumir que $p \in \mathbf{E}^{3}$, e estudar o comportamento destas funçóes altura. Além disso, se $p$ é normal a $M$ em um ponto $Q$, então $Q$ está em $\mathrm{E}^{3}$. Se $Q$ está em $\partial \mathcal{H} S$, então $Q$ também está em $\partial \mathcal{H} M$. Então, reduzimos nosso problema a mostrar que se $Q$ não está em $\partial \mathcal{H} S$, entãp $\ell_{p}$ não tem extremo local em $Q$.

O fecho convexo de $S$ é a união dos fechos convexos das secções horizontais nos planos $z=$ constante. Estas seç̧ões são mostradas na figura 3.2, e os fechos 


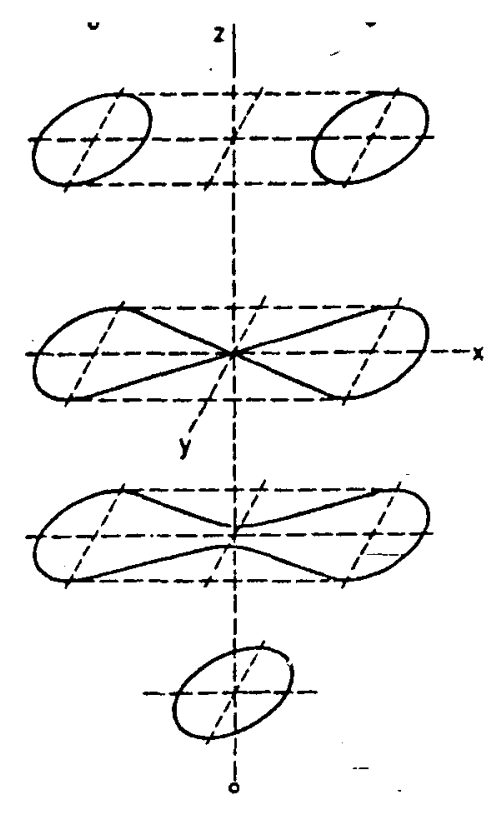

Figura 3.2: Seções

convexos são indicados pelas linhas tracejadas. Suponha que $Q$ é um ponto no nível 2 ou 3 que não está na fronteira do fecho convexo da tal seção. Então $S$ não tem plano suporte local em $Q$, e daí $\ell_{q_{\mid S}}$ tem um ponto crítico de índice 1 ou 2 em $Q$. Logo, $\ell_{q}$ não tem extremo local em $Q$. Suponha agora que $Q$ é um ponto no nível 4 que não está em $\partial \mathcal{H} S$. A curvatura Gaussiana de $S$ neste ponto é positiva e, portanto a restrição de $\ell_{q}$ a $S$ tem um extremo local em $Q$. Entretanto, sob a rotação no $y z$-plano em $\mathbf{E}^{4}$, as duas curvas convexas no nivel 4 percorrem um toro $T$ no $x y w$-espaço o qual não tem plano suporte local em $Q$. Então, ainda neste caso, a função $\ell_{q}$ em $M$ não pode ter extremo local em $Q$. Portanto, todo extremo local de uma função altura não degenerada em $M$ está em $\partial \mathcal{H} M$, e $M$ tem a PDP. Sabemos que $M$ não é 1 -justa pois existem funções altura não degeneradas com pontos críticos de índice 1 enquanto $\beta_{1}\left(S^{3}\right)=0$. 


\section{Capítulo 4}

\section{Top-Sets e Teorema de Chern-Lashof}

Introduziremos na primeira seção a noção de top-set devida a Kuiper, bem como os principais resultados sobre este tópico. Na seção seguinte demonstraremos o teorema de Chern-Lashof-Kuiper para imersões topológicas.

\subsection{Top-sets}

Definição 4.1 Seja $f: X \rightarrow \mathbf{E}^{m}$ uma aplicação continua de um espaço compacto $X$. Para um vetor unitário $p \in \mathrm{E}^{m}$, o top-set de $X$ segundo a direção $p$ é o conjunto

$$
\Omega(p)=\left\{x \in X ; \ell_{p}(x)=\max \ell_{p}(y), y \in X\right\} .
$$

Geometricamente, para um espaço mergulhado $X, \Omega(p)$ é a intersecção de $X$ com o hiperplano $H_{p}$ dado pela equação $\ell p=\max \ell_{p}$. Em termos da aplicação $f$, $\Omega(p)=f^{-1}\left(H_{p}\right)$.

Observação 4.1 Se $f: M \rightarrow \mathbf{E}^{m}$ é uma imersão justa de uma variedade diferenciável e $\ell_{p}$ é não degenerada (portanto polar), entâo $\Omega(p)$ é um ponto simples onde $\ell_{p}$ atinge seu máximo. Entretanto são os top-sets de funções altura degeneradas os mais importantes. 


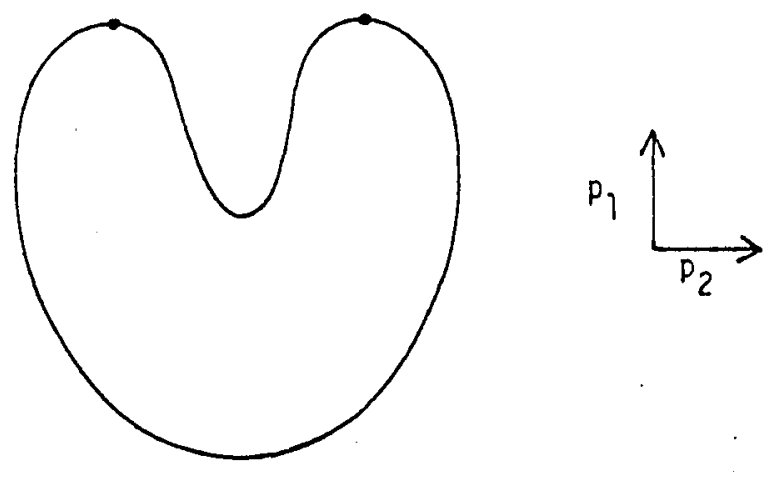

Figura 4.1: Exemplo de top-set

Definição 4.2 Sejam $f: X \rightarrow \mathbf{E}^{m}$ uma aplicação contínua de um espaço compacto e $p_{1}$ e $p_{2}$ vetores ortonormais em $\mathbf{E}^{m} . O 2$-top-set ou top ${ }^{2}$-set $\Omega\left(p_{1}, p_{2}\right)$ é definido por

$$
\Omega\left(p_{1}, p_{2}\right)=\left\{x \in \Omega\left(p_{1}\right) ; \ell p_{2}(x)=\max \ell_{p_{2}}(y), y \in \Omega\left(p_{1}\right)\right\} .
$$

Por indução, para um $k$-sistema ortonormal $p_{1}, \ldots, p_{k}$ definimos o $k$-top-set ou top $^{k}$-set por

$$
\Omega\left(p_{1}, \ldots, p_{k}\right)=\left\{x \in \Omega\left(p_{1}, \ldots, p_{k-1}\right) ; \ell_{p_{k}}(x)=\max \ell_{p_{k}}(y), y \in \Omega\left(p_{1}, \ldots, p_{k-1}\right)\right\} .
$$

Finalmente um top*-set é um top ${ }^{k}$-set para algum $k \geq 1$.

Exemplo 3 Exemplos de top-sets.

a) Seja $f$ um mergulho de $S^{1}$ como a curva plana fechada da figura 4.1. Então, $\Omega\left(p_{1}\right)=\{x, y\}$ e $\Omega\left(p_{1}, p_{2}\right)=\{y\}$.

b) Seja $f: T^{2} \rightarrow \mathbf{E}^{3}$ um mergulho de $T^{2}$ como um toro de revolução. Então o círculo de pontos parabólicos da parte superior do toro é o top-set na direção do vetor $(0,0,1)$.

Apresentaremos a seguir os principais resultados sobre top-sets os quais serão utilizados para resultados posteriores sobre existência de imersões justas para 
variedades não orientáveis e também na demonstração do teorema de ChernLashof. Começamos demonstrando o lema chave. Se $M$ é um espaço topológico compacto e conexo e $f: M \rightarrow \mathbf{E}^{m}$ uma aplicação contínua fixemos as seguintes notações:

$$
M_{r}^{+}(p)=\left\{x \in M ; \ell_{p}(x)>r\right\}
$$

e o seu fecho

$$
\bar{M}_{r}^{+}(p)=\left\{x \in M ; \ell_{p}(x) \geq r\right\}
$$

Lema 4.1 (Lema Chave) Seja $f: M \rightarrow \mathbf{E}^{m}$ uma aplicação contínua definida no espaço topológico compacto $M$. Seja $\Omega$ um top*-set de $f$ e h um semi-espaço fechado em $\mathbf{E}^{m}$ tal que $\Omega \cap f^{-1} h$ está contido em um aberto $U \subset M$. Então existe um vetor unitário $q$ e um número real $r$ tal que

$$
\Omega \cap f^{-1} h \subset M_{r}^{+}(q) \subset \bar{M}_{r}^{+}(q) \subset U .
$$

Além disso, o mesmo se verifica para todo $q^{\prime}$ suficientemente próximo de $q$.

\section{Demonstração:}

O objetivo da demonstração é encontrar um hiperplano $\Pi$ dado por uma equação do tipo $\ell_{q}=r$ tal que

$$
\Omega \cap f^{-1} h \subset M_{r}^{+}(q)
$$

e

$$
M-U \subset M_{r}^{-}(p)=\left\{x \in M ; \ell_{q}(x)<r\right\},
$$

donde teremos a conclusão do lema.

Seja $\Omega$ o top ${ }^{k-1}$-set $\Omega\left(p_{1}, \ldots, p_{k-1}\right)$ e suponha que $h$ é dado pela equação $<x, p_{k}>\geq 0$, isto é, $\ell_{p_{k}} \geq 0$. Podemos escolher a origem em $\mathbf{E}^{m}$ de modo que a função altura determinada por $p_{j}$ tome o valor máximo igual a zero sobre o top $^{j}$-set $\Omega\left(p_{1}, \ldots, p_{j}\right)$ para $1 \leq j \leq k-1$. Então $f\left(\Omega \cap f^{-1} h\right)$ está contido no $(m-(k-1))$-plano $K_{1}$ determinado pelas equações

$$
\ell_{p_{1}}=\ldots=\ell_{p_{k-1}}=0, \ell_{p_{k}} \geq 0 .
$$

Por outro lado, $f\left(M-\left(\Omega \cap f^{-1} h\right)\right)$ está contido no convexo não fechado $K_{2}$ que é a união dos conjuntos $S_{1}, \ldots, S_{k}$ dados pelas equações

$$
\begin{aligned}
& S_{1}: \ell_{p_{1}}<0 \\
& S_{2}: \ell_{p_{1}}=0, \ell_{p_{2}}<0 \\
& \vdots \\
& S_{k}: \ell_{p_{1}}=\ldots=\ell_{p_{k-1}}=0, \ell_{p_{k}}<0 .
\end{aligned}
$$


Os conjuntos convexos $K_{1}$ e $K_{2}$ são disjuntos e $K_{1}$ contém o conjunto convexo e compacto $\mathcal{H}(f(\Omega) \cap h)$ enquanto $K_{2}$ contém o compacto $\mathcal{H} f(M-U)$. Existe um hiperplano $\ell_{q}=r$ que separa os convexos compactos disjuntos $\mathcal{H}(f(\Omega) \cap h)$ e $\mathcal{H} f(M-U)$, e portanto

$$
\Omega \cap f^{-1} h \subset M_{r}^{+}(q) \text { e } M-U \subset M_{r}^{-}(q)
$$

o mesmo se verificando para todo $q^{\prime}$ suficientemente próximo de $q$ com o mesmo valor de $r$.

Corolário 4.1 Seja $f: M \rightarrow \mathbf{E}^{m}$ uma aplicação contínua de um espaço compacto. Se $f$ tem a PDP, entâo todo top*-set de f também tem a PDP.

\section{Demonstração:}

Por indução basta mostrar para top-sets simples. Suponha que o top-set $\Omega(p)$ de $f$ não tem a PDP. Então existe uma função altura $\ell_{z}$ e um número real $\alpha$ tal que o conjunto $\Omega(p) \cap f^{-1}\left(\ell_{z}>\alpha\right)$ é desconexo. Logo para $\beta>\alpha$ pequeno $\Omega(p) \cap f^{-1}\left(\ell_{z} \geq \beta\right)$ tem pelo menos duas componentes conexas $\Omega_{1}$ e $\Omega_{2}$. Consideremos vizinhanças disjuntas $U_{1}$ de $\Omega_{1}$ e $U_{2}$ de $\Omega_{2} \operatorname{com} U=U_{1} \cup U_{2} \subset M$. Apliquemos o lema chave (4.1) para encontrar $q \in \mathbf{E}^{m}$ e $r \in \mathbf{R}$ tal que

$$
\Omega_{1} \cup \Omega_{2} \subset \bar{M}_{r}^{+}(q) \subset U=U_{1} \cup U_{2} .
$$

Claramente, $\bar{M}_{r}^{+}(q)=f^{-1}\left(\ell_{q} \geq r\right)$ não é conexo, e portanto $f$ não tem a PDP.

A seguir provaremos o teorema fundamental de Kuiper que diz que todo topset de um conjunto justo é ainda justo.

Teorema 4.1 (Teorema Fundamental) Seja $f: M \rightarrow \mathbf{E}^{m}$ uma aplicaçâo contínua de um espaço compacto $M$. Se $f$ é justa e $\Omega$ é um top*-set de $f$, então a aplicação $f_{\mid \Omega}$ também é justa.

\section{Demonstração:}

A prova é feita usando a continuidade da homologia de Čech, com a sequência de aproximação sendo construída com o auxílio do lema chave. Por indução, basta mostrarmos para top ${ }^{1}$-sets. A prova será feita somente para o caso em que $f$ é um mergulho e portanto, podemos identificar $M$ com $f(M)$ e simplificar a notação. 
Seja $\Omega$ um top ${ }^{1}$-set de $f$, digamos $\Omega=\Omega(p)$. Pela justeza de $f$ a aplicação

$$
H_{*}\left(\Omega ; \mathbf{Z}_{2}\right) \rightarrow H_{*}\left(M ; \mathbf{Z}_{2}\right)
$$

é injetiva, pois $\Omega=M \cap h^{\prime}$, onde $h^{\prime}$ é o semi-espaço dado pela desigualdade $\ell_{p} \geq \max \ell p$.

Seja $h$ um semi-espaço arbitrário em $\mathbf{E}^{m}$. Devemos mostrar que a aplicação $\alpha: H_{*}\left(\Omega \cap h ; \mathbf{Z}_{2}\right) \rightarrow H_{*}\left(\Omega ; \mathbf{Z}_{2}\right)$ é injetora. Para isto, produziremos uma sequência de semi-espaços $\left\{h_{i}\right\}$ tal que

$$
h_{i} \cap M \supset h_{i+1} \cap M \supset \cdots \supset \bigcap_{j=1}^{\infty}\left(h_{j} \cap M\right)=h \cap \Omega,
$$

usando o lema chave (4.1). Para $i=1$ tome no lema chave $U=M$. Então existem $q_{1} \in S^{m-1}$ e $r_{1} \in \mathbf{R}$ tal que

$$
\Omega \cap h \subset \bar{M}_{r_{1}}^{+}(q) \subset M .
$$

Tomemos então, $h_{1}=\left\{x \in \mathbf{E}^{m} ; \ell_{q_{1}}(x) \geq r_{1}\right\}$. Suponhamos construídos

$$
h_{1} \supset h_{2} \supset \cdots \supset h_{n-1}=M_{r^{\prime}}^{+}\left(q^{\prime}\right) \text {. }
$$

Seja $U=\left\{x \in M_{r^{\prime}}^{+}\left(q^{\prime}\right) ; \operatorname{dist}(x, \Omega \cap h)<1 / n\right\}$. Temos $U \supset \Omega \cap h$ e então pelo lema chave existem $q \in S^{m-1}$ e $r \in \mathbf{R}$ tal que

$$
\Omega \cap h \subset \bar{M}_{r}^{+}(q) \subset U .
$$

Então, tomamos $h_{n}=\left\{x \in \mathbf{E}^{m} ; \ell_{q}(x) \geq r\right\}$ e a sequência $\left\{h_{i}\right\}$ tem a propriedade requerida.

Pela continuidade da homologia de Čech, temos

$$
H_{*}\left(\Omega \cap h ; \mathbf{Z}_{2}\right)=\lim _{i \rightarrow \infty} H_{*}\left(h_{i} \cap \Omega\right) .
$$

Temos o seguinte diagrama comutativo de morfismos em homologia

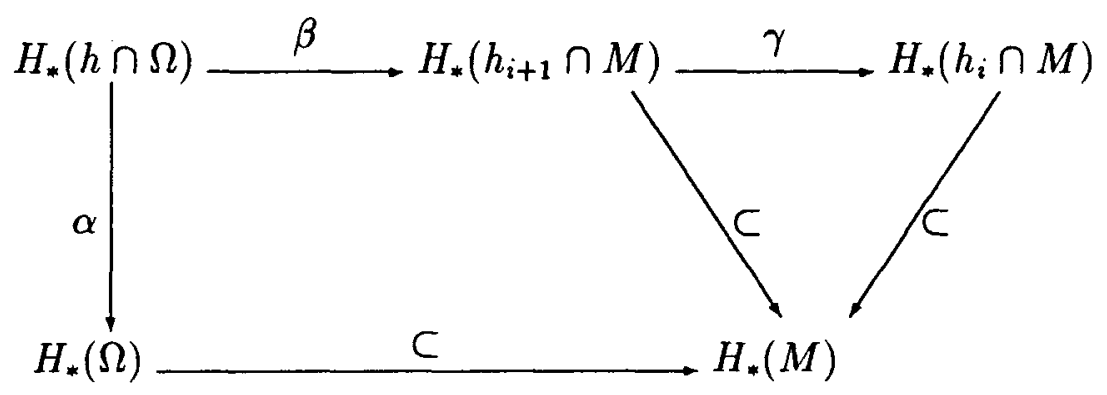


Os morfismos indicados por $\subset$ são injetivos pois $f$ é justa. Segue que toda aplicação da forma $\gamma$ no diagrama é injetiva. Do teorema 3.4 de EilenbergSteenrood ([10]), segue que cada aplicação da forma $\beta$ é injetora. Então, pela comutatividade do diagrama, segue a injetividade de $\alpha$.

Retornemos agora às imersões justas de superfícies em $\mathbf{E}^{3}$.

Definição 4.3 Um meirgulho topológico de um espaço topológico $X \mathrm{em} \mathbf{E}^{m}$ é uma aplicação $f: X \rightarrow \mathbf{E}^{m}$ tal que $f$ é um homeomorfismo de $X$ sobre $f(X)$, considerado como um subespaço de $\mathbf{E}^{m}$.

Definição 4.4 Uma imersâo topológica $f: X \rightarrow \mathbf{E}^{m}$ de um espaço topológico é uma aplicação tal que todo ponto $x \in X$ possui uma vizinhança na qual a restrição de $f$ é um mergulho.

Lema 4.2 Seja $f: X \rightarrow \mathbf{E}^{m}$ uma imersão topológica de um espaço compacto. $O$ conjunto dos pontos em $\mathbf{E}^{m}$ com mais de uma pré-imagem é fechado.

Ver demonstração em [6].

Lema 4.3 Seja $f: X \rightarrow \mathbf{E}^{m}$ uma imersão topológica PDP de um espaço compacto. Seja $\Omega$ um top* -set tal que $f(\Omega)$ é um conjunto convexo. Então $f$ é injetiva em $\Omega$.

\section{Demonstração:}

A prova é por indução sobre a dimensão do convexo $f(\Omega)$. Para $j=0, f(\Omega)$ é um ponto $x$. Como $f$ é imersão e $X$ é compacto, $\Omega$ consiste de um número finito de pontos. Mas $f$ tem a PDP, e portanto $\Omega$ é conexo, isto é, $\Omega$ consiste de um único ponto.

Suponha agora que o lema se verifica para todo top*-set cuja imagem é um conjunto convexo de dimensão menor que $j$. Podemos supor que $f(\Omega)$ é um disco fechado convexo $D$ j-dimensional em um $\mathrm{j}$-plano $\mathbf{E}^{j}$. Note que $\Sigma=\partial D=$ $\bigcup(\Pi \cap D)$ onde $\Pi$ é um plano suporte para $D$ em $\mathbf{E}^{j}$ (ver Lastufka $([19])$ ). Por outro lado cada conjunto $\Pi \cap D$ é igual a $f\left(\Omega^{\prime}\right)$, onde $\Omega^{\prime}$ é um top-set de $\Omega$ de dimensão menor que $j$, e portanto pela hipótese de indução, $f$ é injetiva em $\Omega^{\prime}$. Logo $f$ mergulha $\gamma=f^{-1} \Sigma$ sobre $\Sigma$. Seja

$$
K=\{x \in \Omega ; f(x)=f(y) \text { para algum } y \neq x \in \Omega\} .
$$


Suponhamos por absurdo que $K \neq \emptyset$. Pelo lema 4.2, $K$ é fechado em $X$ e portanto compacto. Desde que $f$ é um mergulho em $\gamma, f\left(K^{\prime}\right)$ e $\mathcal{H} f\left(K^{\prime}\right)$ estão contidos no interior de $D$. O conjunto $\mathcal{H} f(K)$ é um disco convexo fechado de dimensão menor ou igual a $j$. Podemos encontrar $Q \in \partial \mathcal{H} f(K)$ tal que $Q \in f(K)$ e $\mathcal{H} f(K)$ tem um $(j-1)$-plano suporte $\Pi$ em $\mathbf{E}^{j}$. Esta escolha pode ser feita da seguinte maneira: sejam $y \in(f(K))^{c}$ e $Q$ um ponto em $f(K)$ tal que $\operatorname{dist}(Q, y)$ é maximal. Consideramos então $\Pi$ como sendo o hiperplano tangente em $Q$ à esfera com raio $\operatorname{dist}(Q, y)$ centrada em $y$. Como antes $f^{-1}(Q)$ consiste de um número finito de pontos, digamos, $x_{1}, \ldots, x_{l}, \operatorname{com} l \geq 2$. Seja $h$ o semi-espaço fechado limitado por П. Então, $h \cap f(K)=\{Q\}$. Todos os pontos em $h \cap D$ tem exatamente uma pré-imagem exceto $Q$. Logo, $f$ é um homeomorfismo de $V=\Omega \cap f^{-1}(h-\{Q\})$ sobre $(h \cap D)-\{Q\}$. Mostremos que no máximo um ponto em $f^{-1}(Q)$ é ponto limite de $V$. Neste caso, teremos

$$
f^{-1}(f(\Omega) \cap h)=\bar{V} \cup\left\{x_{1}, \ldots, x_{i-1}, x_{i+1}, \ldots, x_{l}\right\},
$$

onde $l \geq 2$, contrariando a PDP de $\Omega$.

Seja $x \in f^{-1}(Q)$ e $W$ uma vizinhança de $x$ em $X$ na qual $f$ é um mergulho. Seja $U=\left(f_{\mid W}\right)^{-1}(B(Q, \epsilon))$ e $S=V \cap f^{-1}(B(Q, \epsilon))$. Note que $S$ é conexo já que $f$ megulha $S$ sobre o conjunto conexo $(h \cap D \cap B(Q, \epsilon))-\{Q\}$. Mostremos que $U \cap S$ é aberto e fechado em $S$. Como $U$ é aberto em $X$ e $S$ tem a topologia relativa é claro que $U \cap S$ é aberto em $S$. Seja $\left\{y_{n}\right\}$ uma sequência em $U \cap S$ convergindo para $y \in S$. Então $\operatorname{dist}(Q, f(y))<\epsilon \mathrm{e}$, para $n$ suficientemente grande, $f\left(y_{n}\right) \in \bar{B}(Q, \delta)$ para algum $\delta<\epsilon$. Então estes $y_{n}$ pertencem ao conjunto

$$
L=\left(f_{\mid W}\right)^{-1}(\bar{B}(Q, \delta)),
$$

que é um subconjuto compacto de $U$. Portanto $y \in L$ e então $y \in U$, provando assim que $U \cap S$ é também fechado em $S$. Como $S$ é conexo segue que se $U \cap S$ é não vazio então $U \cap S=S$.

Consideremos então vizinhanças disjuntas $U_{1}, \ldots, U_{l}$ de $x_{1}, \ldots, x_{l}$ construídas como acima com o mesmo $\epsilon$. Pelo menos um dos pontos em $f^{-1}(Q)$, digamos $x_{1}$, é ponto limite de $V$. De fato, seja $\left\{y_{n}\right\}$ uma sequência em $(h \cap D)-\{Q\}$ que converge para $Q$. Então a sequência $z_{n}=f^{-1}\left(y_{n}\right)$ é uma sequência convergente em $V$. Mas, sendo $f$ um homeomorfismo de $V$ sobre $(h \cap D)-\{Q\}$ devemos ter $\lim z_{n}=x_{i}$ para algum $i$.

Como $U_{1} \cap S$ é não vazio, temos $U_{1} \cap S=S$, e pela conexidade de $S$, segue que $U_{i} \cap S=\emptyset$ para $2 \leq i \leq l$, e portanto $x_{1}$ é o único ponto limite de $V$ em $f^{-1}(Q)$. Assim,

$$
f^{-1}(f(\Omega) \cap h)=\Omega \cap f^{-1} h=\bar{V} \cup\left\{x_{2}, \ldots, x_{l}\right\},
$$




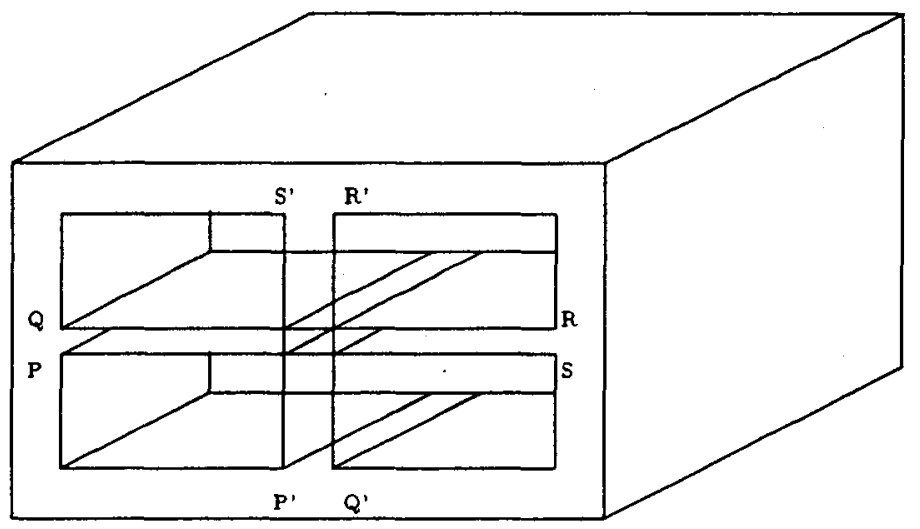

Figura 4.2: Toro retangular de gênus 3

isto é, $f^{-1}(f(\Omega) \cap h)$ é desconexo, contrariando o fato de que $\Omega$ tem a PDP (corolário 4.1).

Observação 4.2 Um top-set de uma imersão diferenciável justa nâo é necessariamente mergulhado. Vejamos um exemplo devido a Banchoff e apresentado por Cecil e Ryan em [6] de um top-set não mergulhado.

Exemplo 4 Top-set não mergulhado.

Começamos com uma versão poliedral. Construamos um toro retangular $T$ a partir de dois anéis idênticos colocados paralelamente ligando-se os pontos correspondentes de suas fronteiras por segmentos de reta.

Fazemos a mesma construção adicionando agora uma faixa retangular com vértices $P, Q, R, S$ transversalmente a cada anel, obtendo um mergulho topológico justo (poliedral) do bitoro. Esta operação pode ser vista como a adição de uma alça (baseada em $P, Q, R, S$ ) ao toro $T$. Em seguida, repetimos o mesmo raciocínio adicionando agora uma nova faixa $P^{\prime}, Q^{\prime}, R^{\prime}, S^{\prime}$ ortogonalmente à primeira, obtendo então uma imersão topológica justa do toro de gênus 3 (figura 4.2). 0 top-set na face da frente não é mergulhado e é dado pela figura 4.3.

Topologicamente, o top-set $\Omega$ é um toro com dois discos removidos. Note que $f(\Omega)$ não é convexo. Os pontos do quadrado central são cobertos duas vezes. 


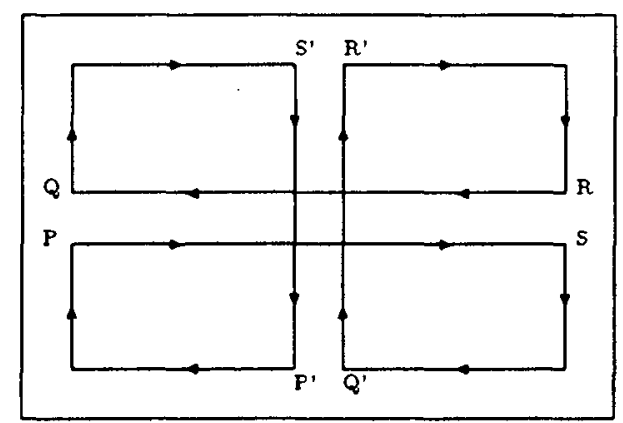

Figura 4.3: Top-set não mergulhado

A fronteira de um disco é mergulhado por $f$ sobre a curva fronteira externa de $f(\Omega)$. A fronteira do outro disco é imersa sobre a curva indicada pelas setas, cujo indice de rotação é 3 . A imagem $f(\Omega)$ é um conjunto plano com a PDP, um disco fechado convexo com 4 discos abertos convexos removidos. Entretanto, se $C$ é a fronteira de um destes discos $f^{-1}(C)$ não é uma curva fechada em $M$. Para obter uma imersão justa diferenciável do toro de gênus 3 com um top-set não mergulhado começamos com o toro justo obtido no exemplo poliedral eliminando as arestas. As alças podem ser suavizadas de forma a terem curvatura Gaussiana não positiva e o top-set frontal ainda tem as mesmas propriedades. A curva com índice de rotação 3 é agora uma curva imersa diferenciável localmente convexa.

Lema 4.4 Seja $f: M \rightarrow \mathbf{E}^{3}$ uma imersão topológica $P D P$ de uma superfície compacta $M$. Para qualquer top*-set $\Omega$,

(a) se $\mathcal{H} f(\Omega)$ tem dimensão 0 ou 1 , então $f$ é um mergulho de $\Omega$ sobre $\mathcal{H} f(\Omega)$.

(b) se $\mathcal{H} f(\Omega)$ tem dimensão 2, então $\partial \mathcal{H} f(\Omega) \subset f(\Omega)$ e f é um mergulho em $\gamma=f^{-1}(\partial \mathcal{H} f(\Omega))$. Além disso, se $\gamma$ separa $M$, então $\Omega$ é o fecho de uma das componentes conexas de $M-\gamma$ ef mergulha $\Omega$ sobre o disco $\mathcal{H} f(\Omega)$.

\section{Demonstração:}

(a) Se $\mathcal{H} f(\Omega)$ tem dimensão 0 , então $f$ é injetiva em $\Omega$, pelo lema 4.3. Suponha agora, que $\mathcal{H} f(\Omega)$ é o segmento $\left[Q_{1}, Q_{2}\right]$ em $\mathbf{E}^{3}$. Como todo ponto 
em $\mathcal{H} f(\Omega)$ é uma combinação convexa de pontos em $f(\Omega)$, os pontos $Q_{1}$ e $Q_{2}$ estão em $f(\Omega)$. Desde que $\Omega$ é conexo, o conjunto conexo $f(\Omega)$ deve ser todo o segmento $\left[Q_{1}, Q_{1}\right]$, e então, pelo lema $4.3 f$ é injetiva.

(b) Suponhamos que $\mathcal{H} f(\Omega)$ está no 2 -plano $\mathbf{E}^{2}$. Toda reta suporte $\ell$ de $\mathcal{H} f(\Omega)$ é reta suporte de $f(\Omega)$. Para uma tal reta suporte $\ell$, o conjunto $\Omega^{\prime}=$ $f^{-1}(\ell) \cap \Omega$ é um top-set de $\Omega$ e $\mathcal{H} f\left(\Omega^{\prime}\right)$ tem dimensão 0 ou 1. Pela parte (a), segue que $f$ é um mergulho de $\Omega^{\prime}$ sobre $\mathcal{H} f\left(\Omega^{\prime}\right)=\ell \cap \mathcal{H} f(\Omega)$. Por outro lado, $\partial \mathcal{H} f(\Omega)$ é a união disjunta destas interseç̧ões $\ell \cap \mathcal{H} f(\Omega)$, onde $\ell$ é uma reta suporte de $f(\Omega)$ em $\mathbf{E}^{2}$. Assim,

$$
\begin{aligned}
\partial \mathcal{H} f(\Omega) & =\bigcup_{\ell}(\ell \cap \mathcal{H} f(\Omega)) \\
& =\bigcup_{\Omega^{\prime}} \mathcal{H} f\left(\Omega^{\prime}\right) \\
& =\bigcup f\left(\Omega^{\prime}\right) \subset f(\Omega),
\end{aligned}
$$

e $f$ é um mergulho em $\gamma=f^{-1}(\partial \mathcal{H} f(\Omega))$.

Suponhamos agora, que $\gamma$ separa $M$ e que a equação do plano de $f(\gamma)$ é $\ell_{p}=r$. Então, uma das componentes de $M-\gamma$, que chamaremos $V$, é aplicada dentro do disco convexo $\mathcal{H} f(\Omega)$. De fato, se existir $x \in V$ tal que $f(x) \notin \mathcal{H} f(\Omega)$ então $\ell_{p}(x)<r$, isto é, $x \in M_{r}^{-}(p)$. Mas, se $y \in M-\bar{V}$ então $\ell_{p}(y)<r$, e portanto, $y \in M_{\tau}^{-}(p)$. Logo $M_{\tau}^{-}(p)$ é desconexo, contrariando a PDP.

Como $f$ é uma imersão, pelo Teorema da Invariância do Domínio de Brower temos que $f(V)$ é um subconjunto aberto do disco convexo aberto $\operatorname{int}(\mathcal{H} f(\Omega))$. Mostremos que $f(V)$ é também fechado em $\operatorname{int}(\mathcal{H} f(\Omega))$. Seja $Q$ um ponto limite de $f(V)$ no interior de $\mathcal{H} f(\Omega)$ e $\left\{Q_{n}\right\}$ uma sequência em $f(V)$ convergindo para $Q$. Seja $x_{i} \in V$ tal que $f\left(x_{i}\right)=Q_{i}$. A sequência $\left\{x_{i}\right\}$ tem um ponto limite $x$ no compacto $\bar{V} \cup \gamma$. Por continuidade $f(x)=Q$ e $x$ não pode estar em $\gamma$, desde que $f(\gamma)$ e $\operatorname{int}(\mathcal{H} f(\Omega))$ são disjuntos. Então $x \in V$ e portanto $Q \in f(V)$ e, então $f(V)$ é fechado em $\operatorname{int}(\mathcal{H} f(\Omega))$. Logo, $f(V)=\operatorname{int}(\mathcal{H} f(\Omega))$ e $f(\Omega)$ é convexo. Pelo lema $4.3, f$ mergulha $\Omega$ sobre o conjunto convexo $\mathcal{H} f(\Omega)$ e $\Omega=\bar{V}$.

Como consequência do lema daremos a prova de Kuiper do Teorema de ChernLashof em $\mathbf{E}^{3}$.

Teorema 4.2 Seja $f: S^{2} \rightarrow \mathbf{E}^{3}$ uma imersão topológica justa. Então $f$ mergulha $S^{2}$ sobre a superficie convexa $\partial \mathcal{H} f\left(S^{2}\right)$.

\section{Demonstração:}


Como antes $\partial \mathcal{H} f\left(S^{2}\right)=\bigcup_{\Omega} \mathcal{H} f(\Omega)$, onde $\Omega$ varia sobre todos os top-sets de $f$. Como toda curva fechada $\gamma$ separa $S^{2}$, o lema anterior assegura que $f$ mergulha cada top-set $\Omega$ sobre $\mathcal{H} f(\Omega)$. Logo, $f^{-1}\left(\partial \mathcal{H} f\left(S^{2}\right)\right)$ é uma 2-esfera mergulhada em $S^{2}$ e portanto deve ser igual a $S^{2}$.

\subsection{O Teorema de Chern-Lashof}

Em 1957, Chern e Lashof [8] provaram que se $f: S^{n} \rightarrow \mathbf{E}^{m}$ é uma imersão diferenciável com curvatura total absoluta mínima então $f$ mergulha $S^{n}$ como uma hipersuperfície convexa em um subespaço $\mathbf{E}^{n+1}$ de $\mathbf{E}^{m}$. Em 1970, Kuiper [12] obteve o mesmo resultado supondo $f$ apenas imersão topológica justa e, em 1979 , ele generalizou o resultado mostrando que se $f: S^{n} \rightarrow \mathbf{E}^{m}$ é uma aplicação contínua substancial e justa, então $m=n+1$ e $f\left(S^{n}\right)=\partial \mathcal{H} f\left(S^{n}\right)$ é a fronteira de um corpo convexo $(n+1)$-dimensional. Antes de apresentarmos a prova de Kuiper deste teorema para imersões topológicas precisamos de algumas definições.

Definição $4.5 U m$ top ${ }^{*}$-set é dito um $\mathbf{E}^{j}$-top-set se sua imagem está contida em um $\mathbf{E}^{j}$-plano e não está contida em nenhum $\mathbf{E}^{j-1}$-plano.

Definição 4.6 Um espaço topológico $X$ conexo por caminhos é dito k-conexo, $k \geq 1$, se os seus grupos de homotopia $\Pi_{i}(X)$ são todos nulos para $1 \leq i \leq k$. 0-conexo significa conexo por caminhos.

O lema abaixo é a principal ferramenta topológica na demonstração do teorema de Chern-Lashof-Kuiper. Sua demonstração pode ser encontrada em [6].

Lema 4.5 Seja $f: M \rightarrow \mathbf{E}^{m}$ uma imersão topológica de uma variedade compacta $M$. Assuma que para quase todos os vetores unitários $p \in S^{m-1}$ o conjunto $M_{r}(p)$ é $k$-conexo para todo $r \in \mathbf{R}$. Então para qualquer $\mathbf{E}^{j}-$ top* ${ }^{*}$-set $\Omega$, tem-se:

(a) se $j \leq k+1$, então $f$ é um mergulho de $\Omega$ sobre $\mathcal{H} f(\Omega)$.

(b) se $j=k+2$, então $\partial \mathcal{H} f(\Omega) \subset f(\Omega)$ e, $f$ é um mergulho em $\gamma=f^{-1}(\partial \mathcal{H} f(\Omega))$.

A prova do teorema de Chern-Lashof-Kuiper será feita para imersões fracamente não degeneradas que passamos a definir agora. 
Definição 4.7 Uma função continua a valores reais $\phi: M \rightarrow \mathbf{R}$ definida na variedade topológica compacta $M$ é dita não crítica em $x \in M$ se existe uma vizinhança coordenada de $x$ para a qual $\phi$ é uma das coordenadas. Do contrário $x$ é dito um ponto crítico de $\phi$.

Definição 4.8 Uma imersão topológica $f: M \rightarrow \mathbf{E}^{m}$ é dita fracamente não degenerada se quase toda funçấo altura linear $\ell_{p}$ tem pontos críticos isolados em $M$.

Com a ajuda da teoria de pontos críticos é possível mostrar que se $f: S^{n} \rightarrow \mathbf{E}^{m}$ é justa e fracamente não degenerada, então quase toda função altura tem exatamente dois pontos críticos. Podemos então apresentar a versão topológica do teorema de Chern-Lashof-Kuiper.

Teorema 4.3 (Chern-Lashof-Kuiper) Seja $f: S^{n} \rightarrow \mathbf{E}^{m}$ uma imersão topológica substancial tal que quase todas as funções alturas lineares têm exatamente 2 pontos críticos. Então $m=n+1$ e $f$ mergulha $S^{n}$ como uma hipersuperfície convexa.

\section{Demonstração:}

Afirmamos que se $\ell_{p}$ tem exatamente dois pontos críticos, então $M_{r}(p)$ é $(n-1)$-conexo.

De fato, suponhamos que $\ell_{p}$ tenha um valor mínimo $a$ em um ponto crítico $x_{1}$ e um valor máximo $b$ em $x_{2}$. Se $a<r<b$, então existe um retrato por deformação de $M_{r}(p)$ sobre $M_{a}(p)=\left\{x_{1}\right\}$. Então $M_{r}(p)$ é claramente $(n-$ 1)-conexo, enquanto que se $r \geq b, M_{r}(p)=S^{n}$.

Suponhamos agora que exista um $\mathbf{E}^{n+1}$-top*-set $\Omega$. Pela parte (b) do lema 4.5, $\gamma=f^{-1}(\partial \mathcal{H} f(\Omega))$ é uma $n$-esfera mergulhada em $S^{n}$. Então $\gamma=S^{n}$ e $f$ mergulha $S^{n}$ como a hipersuperfície convexa $\partial \mathcal{H} f\left(S^{n}\right)$. Além disso, sendo $f$ substancial $\mathbf{E}^{n+1}=\mathbf{E}^{m}$.

Suponhamos agora que todos os top ${ }^{1}$-sets de $f$ são $\mathbf{E}^{j}$-top-sets, com $j \leq n$. Então a parte (a) do lema 4.5 se aplica a cada top ${ }^{1}$ - set, isto é, $f$ mergulha cada top $^{1}-$ set $\Omega$ sobre $\mathcal{H} f(\Omega)$. Então, como

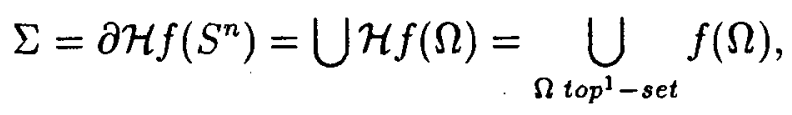


segue que $f\left(S^{n}\right)$ contém a $(m-1)$-esfera $\Sigma$. Além disso, $\gamma=f^{-1} \Sigma$ é uma $(m-1)$-esfera mergulhada em $S^{n}$. Logo, $m \leq n+1$. Mas, por outro lado, $m \geq n+1$ pois $f$ é uma imersão. Assim, vemos que $m=n+1$ e $f$ mergulha $S^{n}$ sobre $\Sigma$.

A prova estará completa se mostrarmos que não existem $\mathbf{E}^{j}$-top*-sets com $j>n+1$. Se existir um tal top*-set repetindo o processo de tomar top-sets podemos eventualmente produzir um $\mathbf{E}^{n+1}-$ top $^{*}$-set ou um $\mathbf{E}^{q}$-top ${ }^{*}$-set $\Omega$ com $q>n+1$ tal que todos os top ${ }^{1}$-sets de $\Omega$ sejam $\mathbf{E}^{i}$-top*-sets com $i \leq n$. Neste último caso aplicando o argumento acima obtemos que $S^{n}$ contém a $(q-1)$-esfera $f^{-1}(\partial \mathcal{H} f(\Omega))$. Então, $q \leq n+1$, uma contradição. No primeiro caso já haviamos obvservado que a existência de um $\mathbf{E}^{n+1}$ - top*-set implica a conclusão do teorema. Portanto, podemos concluir a não existência de $\mathbf{E}^{j}$-top ${ }^{*}$-sets $\operatorname{com} j>n+1$, e o teorema está provado.

Observação 4.3 Uma imersão $C^{2}$ é fracamente não degenerada $e$, portanto a versão diferenciável do teorema segue da versão acima. 


\section{Capítulo 5}

\section{Superfícies Justas em $\mathbf{E}^{3}$}

Começamos este capítulo apresentando os exemplos de Kuiper de imersões justas de superfícies em $\mathbf{E}^{3}$. Kuiper produziu exemplos de mergulhos suaves justos de todas as supefícies orientáveis em $\mathbf{E}^{3}$ ([14]) e imersões suaves justas de superfícies não orientáveis com característica de Euler $\chi<-1$ ([15]). Mostrou também que não existem imersões justas, sequer continuas, do plano projetivo $\mathbf{P}^{2}$ ou da garrafa de Klein $K^{2}$ em $\mathbf{E}^{3}$. Recentemente, F. Haab ([11]) provou a não existência de imersões justas no caso $M=P^{2} \# T^{2}(\chi=-1)$.

\subsection{Superfícies Justas}

Já conhecemos um mergulho justo de $S^{2}$ em $\mathbf{E}^{3}$, a saber, as superfícies convexas. Vejamos um mergulho justo do toro $T^{2} \mathrm{em} \mathbf{E}^{3}$. Antes façamos a seguinte observação.

Observação 5.1 Sejam $f: X \rightarrow \mathbf{E}^{m}$ e $g: Y \rightarrow \mathbf{E}^{k}$ duas aplicações justas. Então a aplicaçâao $f \times g: X \times Y \rightarrow \mathbf{E}^{m+k}$ também é justa. A demonstração deste fato pode ser encontrado em [6].

Exemplo 5 Mergulho Justo do Toro $T^{2}$.

Seja $f: S^{1} \rightarrow \mathbf{E}^{2}$ um mergulho justo. Então, pela observação acima

$$
g=f \times f: S^{1} \times S^{1}=T^{2} \rightarrow \mathbf{E}^{4}
$$


dada por

$$
g(\theta, \varphi)=(f(\theta), f(\varphi))
$$

é um mergulho justo de $T^{2}$ em $\mathbf{E}^{4}$. Tomemos agora $r=\sqrt{2}$. Então, vemos que $S^{1} \times S^{1} \subset S^{3}(r)$. Logo, $g$ é um mergulho justo de $T^{2}$ em $S^{3}(r)$. Seja $q=$ $(0,0,0, r) \in S^{3}(r)$, e considere a projeção estereográfica por $q P_{q}: S^{3}(r) \rightarrow \mathbf{E}^{3}$. Então, $P_{q} \circ g: T^{2} \rightarrow \mathbf{E}^{3}$ é um mergulho justo de $T^{2}$ em $\mathbf{E}^{3}$. De fato, como $q \notin g\left(T^{2}\right)$, então $\left(P_{q} \circ g\right)\left(T^{2}\right)$ é compacto e difeomorfo a $T^{2}$, isto é, $P_{q} \circ g$ é um mergulho de $T^{2}$ em $\mathbf{E}^{3}$. Seja $h$ um semi-espaço fechado em $\mathbf{E}^{3}$, determinado por um hiperplano ПI. Então

$$
\left(P_{q} \circ g\right)^{-1}(h)=g^{-1}\left(P_{q}^{-1} h\right) .
$$

Vejamos o que $P_{q}^{-1} h$ representa em $S^{3}(r)$.

O hiperplano $\Pi$ é representado por uma esfera $S^{2}$ em $S^{3}(r)$. Então, $P_{q}^{-1} h$ é uma bola fechada na métrica de $S^{3}(r)$. Logo, $P_{q}^{-1} h=S^{3}(r) \cap H$, onde $H$ é um semi-espaço fechado conveniente em $\mathrm{E}^{4}$. A justeza de $P_{q} \circ g$ segue então da justeza de $g$. Só para exemplificar, tome $f: S^{1} \rightarrow \mathbf{E}^{2}$ dada por $f(\theta)=(\cos (\theta), \sin (\theta))$. Então o mergulho justo de $T^{2}$ em $\mathbf{E}^{3}$ é dado por

$$
(\theta, \varphi) \rightarrow \sqrt{2}\left(\frac{\cos (\theta)}{\sqrt{2}-\operatorname{sen}(\varphi)}, \frac{\operatorname{sen}(\theta)}{\sqrt{2}-\operatorname{sen}(\varphi)}, \frac{\cos (\varphi)}{\sqrt{2}-\operatorname{sen}(\varphi)}\right)
$$

Antes de apresentarmos os exemplos de Kuiper introduziremos duas construções básicas: uma superfície convexa com partes planares e uma alça de curvatura negativa que pode ser colada diferenciavelmente às partes planares da tal superfície convexa.

Para isto, consideremos inicialmente a curva (figura 5.1)

$$
z= \begin{cases} \pm 1, & 0 \leq x \leq 1 \\ \pm \sqrt{1-(1-x)^{2}}, & 1 \leq x \leq 2\end{cases}
$$

que se rotacionada em torno do eixo $O z$ produz uma superfície convexa $S$ com duas partes planares paralelas. Por outro lado, a rotação em torno do eixo $\mathrm{Oz}$ do semi-círculo

$$
x=2-\sqrt{1-z^{2}},|z| \leq 1
$$

fornece uma alça de curvatura negativa.

Removendo dois discos da superfície $S$, sendo um em cada parte planar podemos adicionar a alça $H$ de curvatura negativa sem destruir a diferenciabilidade. 


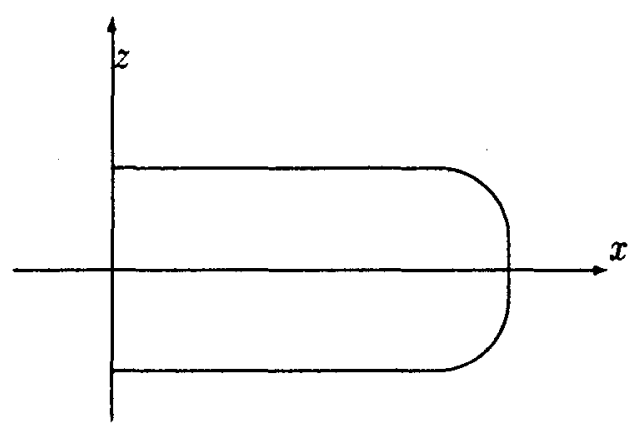

Figura 5.1:

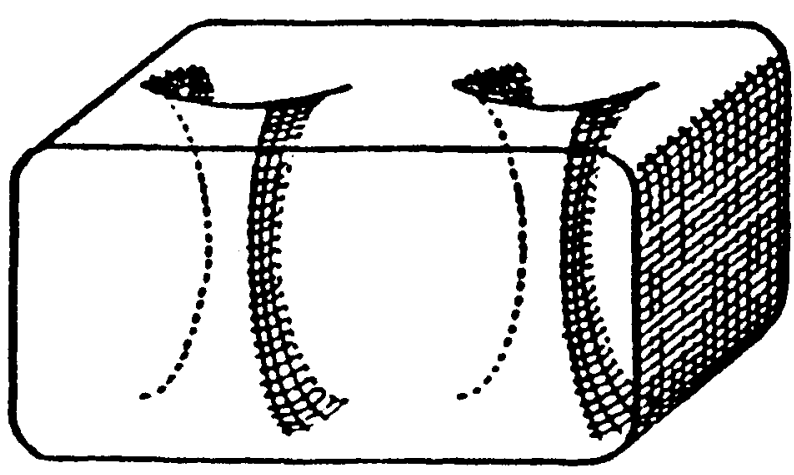

Figura 5.2: Bitoro Justo

Pelo teorema 1.9 nenhuma função altura pode ter extremo local sobre uma superfície de curvatura negativa. Portanto, pelo corolário 2.2, se a superfície original $S$ é justa, também será justa a superfície resultante, isto é, toda função altura não degenerada é ainda polar.

Estamos agora em condições de apresentar os exemplos de Kuiper de superfícies justas.

Exemplo 6 Mergulhos justos de superfícies orientáveis.

Começamos com uma superfície convexa $M$ que tem duas partes planares paralelas. Para construir uma superfície orientável justa de genus $\mathrm{g}$, removemos $\mathrm{g}$ discos circulares idênticos das partes planas do topo e da base. Em seguida unimos cada par de discos por uma alça de curvatura Gaussiana negativa (figura $5.2)$. 


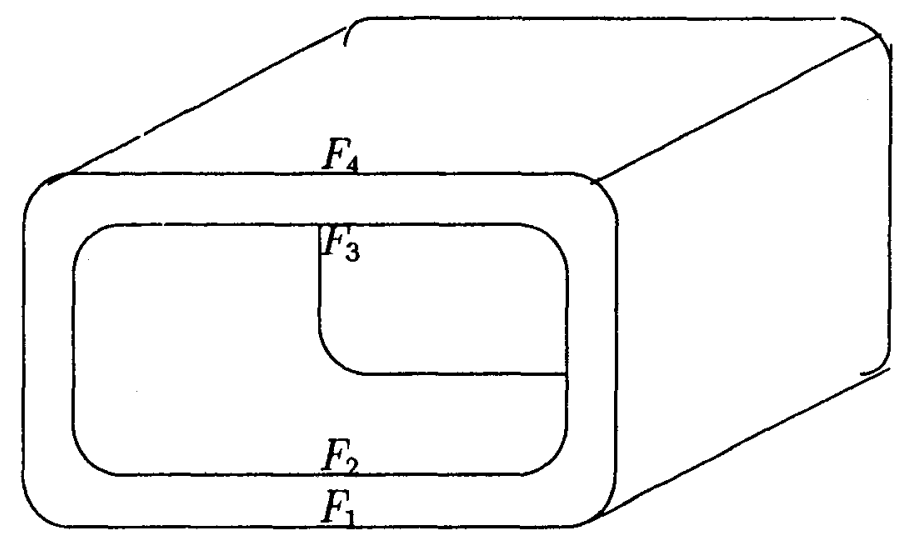

Figura 5.3: Toro justo de Kuiper

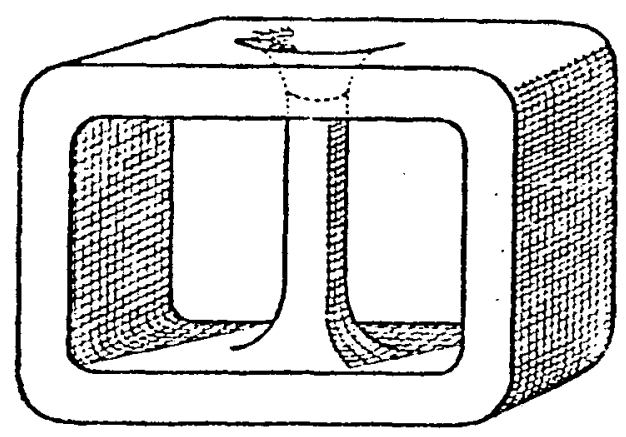

Figura 5.4: Imersão justa de $K^{2} \# T^{2}$

Construção alternativa: Começamos com o toro justo de Kuiper mostrado na figura 5.3 com quatro faces planares horizontais $F_{1}, F_{2}, F_{3}, F_{4}$ em ordem ascendente de altura. Um mergulho justo de uma superfície orientável de genus $g$, $g \geq 2$, é obtido adicionando-se $g-1$ alças de curvatura negativa de $F_{2}$ a $F_{3}$. Uma imersão justa que não é mergulho é obtida acrescentando-se $g-1$ alças de $F_{1}$ a $F_{4}$, cortando as faces $F_{2}$ e $F_{3}$. A construção de uma imersão justa que não é um mergulho para o toro $(g=1)$ não é tão trivial e pode ser encontrada em ([18]).

Exemplo 7 Imersões justas de superfícies não orientáveis com $\chi$ par.

Este exemplo é uma pequena variação do anterior. Começamos com o toro justo da figura 5.3. Uma imersão justa da Garrafa de Klein com uma alça (figura 5.4), $K^{2} \# T^{2}$, é obtida removendo-se discos circulares de $F_{2}$ e $F_{4}$ e, em seguida unindo as fronteiras destes discos por uma alça de curvatura negativa que intercepta a face $F_{3}$. 
Para obtermos uma imersão justa de uma superfície não orientável com característica de Euler $\chi=-2 k, k>1$, adicjonamos $k-1$ alças de $F_{2}$ a $F_{3}$ à superfície justa $K^{2} \# T^{2}$.

\section{Exemplo 8 Superfície de Veronese.}

Seja $S^{2}$ a esfera em $\mathbf{E}^{3}$ dada por

$$
x^{2}+y^{2}+z^{2}=1
$$

e $\phi: S^{2} \rightarrow \mathbf{E}^{6}$ dada por

$$
\phi(x, y, z)=\left(x^{2}, y^{2}, z^{2}, \sqrt{2} y z, \sqrt{2} z x, \sqrt{2} x y\right) .
$$

Em pontos antípodas $\phi$ assume o mesmo valor. Então ela induz uma aplicação $\varphi: \mathbf{P}^{2} \rightarrow \mathbf{E}^{6}$, isto é, $\phi=\varphi \circ \pi$ onde $\pi: S^{2} \rightarrow \mathbf{P}^{2}$ é a projeção canônica. Mostremos que $\varphi$ é uma imersão. Considere as coordenadas de $\mathbf{P}^{2}$ dadas por $(w, t) \rightarrow\left(D_{1}, w, t\right)$ onde $D_{1}=\sqrt{1-w^{2}-t^{2}}$ e $(w, t) \in U=\left\{(w, t) ; w^{2}+t^{2}<1\right\}$. Então

$$
\varphi(w, t)=\phi\left(D_{1}, w, t\right)=\left(1-w^{2}-t^{2}, w^{2}, t^{2}, \sqrt{2} w t, \sqrt{2} D_{1} t, \sqrt{2} D_{1} w\right)
$$

e sua jacobiana é dada por

$$
J(\varphi)=\left(\begin{array}{cc}
-2 w & -2 t \\
2 w & 0 \\
0 & 2 t \\
\sqrt{2} t & \sqrt{2} w \\
-\frac{\sqrt{2} t w}{D_{1}} & \sqrt{2} D_{1}-\frac{\sqrt{2} t^{2}}{D_{1}} \\
\sqrt{2} D_{1}-\frac{\sqrt{2} w^{2}}{D_{1}} & -\frac{\sqrt{2} t w}{D_{1}}
\end{array}\right)
$$

Esta matriz tem posto 2 sempre que $w \neq 0$ ou $t \neq 0$. Logo, $\varphi_{*}: T_{p} \mathbf{P}^{2} \rightarrow \mathbf{E}^{6}$ é injetora para todo $p \in \mathbf{P}^{2}-\left\{\left[D_{1}, 0,0\right]=[1,0,0]\right\}$, e portanto é injetora para todo $p$ no sistema de coordenadas $U$. Analogamente, tomando os sistemas de coordenadas $(w, t) \rightarrow\left(w, D_{1}, t\right)$ e $(w, t) \rightarrow\left(w, t, D_{1}\right)$ segue que $\varphi_{*}: T_{p} \mathbf{P}^{2} \rightarrow \mathbf{E}^{6}$ é injetora para todo $p \in \mathbf{P}^{2}$. Logo, $\varphi$ é imersão. Como $\mathbf{P}^{2}$ é compacto e $\varphi$ é injetiva segue que $\varphi$ é um mergulho.

Sejam $\left(u_{1}, \ldots, u_{6}\right)$ as coordenadas canônicas de $\mathbf{E}^{6}$. Como $x^{2}+y^{2}+z^{2}=1$ segue que $\varphi\left(\mathbf{P}^{2}\right)$ está no subespaço

$$
\mathbf{E}^{5}=\left\{\left(u_{1}, \ldots, u_{6}\right) ; u_{1}+u_{2}+u_{3}=1\right\} .
$$


Suponha que $\varphi\left(\mathbf{P}^{2}\right)$ está contido no 4-espaço

$$
\left\{\begin{array}{l}
u_{1}+u_{2}+u_{3}=1 \\
\sum_{i=1}^{6} a_{i} u_{i}=a
\end{array}\right.
$$

Então para todo $(x, y, z) \in S^{2}$ temos

$$
\left\{\begin{array}{l}
x^{2}+y^{2}+z^{2}=1 \\
a_{1} x^{2}+a_{2} y^{2}+a_{3} z^{3}+a_{4} \sqrt{2} y z+a_{5} \sqrt{2} x z+a_{6} \sqrt{2} x y=a .
\end{array}\right.
$$

Tomando então os pontos

$$
\begin{gathered}
(1,0,0),(0,1,0),(0,0,1) \\
\left(\frac{\sqrt{2}}{2}, \frac{\sqrt{2}}{2}, 0\right),\left(\frac{\sqrt{2}}{2}, 0, \frac{\sqrt{2}}{2}\right),\left(0, \frac{\sqrt{2}}{2}, \frac{\sqrt{2}}{2}\right)
\end{gathered}
$$

obtemos $a_{1}=a_{2}=a_{3}=a$ e $a_{4}=a_{5}=a_{6}=0$. Então $\varphi\left(\mathbf{P}^{2}\right)$ não está contido em nenhum $\mathbf{E}^{4}$, isto é, $\varphi$ é substancial.

Note que

$$
\left(x^{2}\right)^{2}+\left(y^{2}\right)^{2}+\left(z^{2}\right)^{2}+2\left(y^{2} z^{2}+z^{2} x^{2}+x^{2} y^{2}\right)=\left(x^{2}+y^{2}+z^{2}\right)^{2}=1,
$$

isto é, $\varphi\left(\mathbf{P}^{2}\right)$ está contido na esfera $S^{5}$ em $\mathbf{E}^{6}$ de equação $u_{1}^{2}+\cdots+u_{6}^{2}=1$. Então, $\varphi$ é um mergulho de $\mathbf{P}^{2}$ na $4-$ esfera $S^{4}=S^{5} \cap \mathbf{E}^{5}$.

Seja II um hiperplano de $\mathbf{E}^{5}$ dado pela equação

$$
\sum_{i=1}^{6} a_{i} u_{i}=c
$$

Se $\varphi(x, y, z) \in \Pi$, então

$$
a_{1} x^{2}+a_{2} y^{2}+a_{3} z^{2}+a_{4} \sqrt{2} y z+a_{5} \sqrt{2} x z+a_{6} \sqrt{2} x y=c,
$$

isto é, II corta $\mathbf{P}^{2}$ em uma cônica. Tal cônica separa $\mathbf{P}^{2}$ em no máximo duas componentes conexas. Então $\varphi$ tem a propriedade dos dois pedaços, e como $\mathbf{P}^{2}$ tem dimensão 2, $\varphi$ é justa. $\varphi$ é o mergulho de Veronese de $\mathbf{P}^{2}$ em $\mathbf{E}^{5} \mathrm{em}$ codimensão máxima (teorema 3.5).

Para obter um mergulho substancial justo de $\mathbf{P}^{2}$ em $\mathbf{E}^{4}$ consideremos o 4-espaço $\mathbf{E}^{4}$ gerado pelos vetores

$$
\frac{e_{1}-e_{2}}{\sqrt{2}}, e_{5}, e_{6}, e_{4}
$$


onde $\left\{e_{1}, \ldots e_{6}\right\}$ é a base canônica de $\mathbf{E}^{6}$ e projetamos $\varphi$ ortogonalmente sobre este subespaço. Temos

$$
\mathbf{E}^{6}=\mathbf{E}^{4} \oplus \mathbf{E}^{2}
$$

onde $\mathbf{E}^{2}$ é o 2-espaço gerado pelos vetores $\frac{e_{1}+e_{6}}{\sqrt{2}}, e_{3}$. Nesta base, $\varphi$ tem a seguinte expressão

$$
\varphi(x, y, z)=\left(\frac{x^{2}-y^{2}}{\sqrt{2}}, \sqrt{2} x z, \sqrt{2} x y, \sqrt{2} y z, \frac{x^{2}+y^{2}}{\sqrt{2}}, z^{2}\right) .
$$

Então a projeção de $\varphi$ sobre $\mathbf{E}^{4}$ define um mergulho de $\mathbf{P}^{2}$ em $\mathbf{E}^{4}$ dado por

$$
f:(x, y, z) \rightarrow\left(\frac{x^{2}-y^{2}}{\sqrt{2}}, \sqrt{2} x z, \sqrt{2} x y, \sqrt{2} y z\right) .
$$

Este mergulho é justo, pois justeza é preservada por projeção ortogonal.

\subsection{Top-Ciclos}

A partir de agora estebeleceremos resultados que permitirão verificar a existência ou não de imersões justas do Plano Projetivo e da Garrafa de Klein em $\mathbf{E}^{3}$.

Do lema 4.4 segue que se $\mathcal{H} f(\Omega)$ é 2 -dimensional e $\mathcal{H} f(\Omega) \neq f(\Omega)$, então a curva $\gamma=f^{-1}(\partial \mathcal{H} f(\Omega))$ não separa $M$. Em outras palavras, a curva $\gamma$ é não bordante em $M$, ou seja, $\gamma$ representa uma 1-classe essencial de homologia.

Definição 5.1 Uma tal curva será dita um top-ciclo de $f$.

As curvas de pontos parabólicos do topo e da base do toro de revolução são top-ciclos.

Um primeiro resultado sobre top-ciclos é o seguinte

Lema 5.1 Seja $f: M \rightarrow \mathbf{E}^{3}$ uma imersão topológica justa de uma superfície compacta e conexa. Entâo o número de top-ciclos é finito.

\section{Demonstração:}


A prova consiste em mostrar que o conjunto de pontos $p \in S^{2}$ tajs que $\Omega(p)$ contém um top-ciclo é isolado e portanto finito. Suponha que $\Omega(p)$ contém um top-ciclo. Como $f$ é injetiva em $\gamma=f^{-1}(\partial \mathcal{H} f(\Omega(p)))$, pelo lema 4.2, existe $\epsilon>0$ tal que $f$ restringe-se a um mergulho na imagem inversa da vizinhança tubular de raio $\epsilon$ ao redor de $f(\gamma)$. Seja $U$ uma vizinhança anular de $\gamma$ a qual é mergulhada dentro deste tubo. Então, pondo $W=\Omega(p) \cap U$, temos $W \subset U \subset \bar{U} \subset M$ com $\ell_{p}(x)=\max \ell_{p}, x \in W$ e $\ell_{p}(x)<\max \ell_{p}$ se $x \in \bar{U}-W$. Então, pelo lema 3.1 existe uma vizinhança $N$ de $p$ em $S^{2}$ tal que se $q \in N$, então $\Omega(q) \subset U$. Mostremos que para todo $q \in N, \Omega(q)$ é homologicamente trivial.

Seja $q \in N$ e escreva $q=\cos (\alpha) p+\operatorname{sen}(\alpha) p^{\prime}$, onde $p^{\prime}$ é ortogonal a $q$ e $\operatorname{sen}(\alpha)>0$. Seja $r$ o valor de $\ell_{p^{\prime}}$ no top ${ }^{2}$-set $\Omega\left(p, p^{\prime}\right)$. Sejam $\beta$ o máximo absoluto de $\ell_{p}$ em $M$ e $\gamma$ o máximo absoluto de $\ell_{q}$ em $M$. Então, se $x \in \Omega(q)$ temos

$$
\ell p^{\prime}(x)=\frac{\ell_{q}(x)}{\operatorname{sen}(\alpha)}-\tan (\alpha) \ell_{p}(x) \leq \frac{\gamma}{\operatorname{sen}(\alpha)}-\tan (\alpha) \beta
$$

Como $r=\max \left\{\ell_{p^{\prime}}(x) ; x \in \Omega(p)\right\}$ segue que, $\frac{\gamma}{\operatorname{sen}(\alpha)}-\tan (\alpha) \beta \geq r$. Assim, se $x \in \Omega(q)$ temos

$$
\ell_{p^{\prime}}(x)=\frac{\gamma}{\operatorname{sen}(\alpha)}-\tan (\alpha) \ell_{p}(x) \geq \frac{\gamma}{\operatorname{sen}(\alpha)}-\tan (\alpha) \beta \geq r .
$$

Logo, $\Omega(q)$ está contido na intersecção de $U$ com o semi-espaço fechado $\ell_{p^{\prime}} \geq r$. Portanto, $\Omega(q)$ está contido em uma vizinhança contrátil de $\Omega\left(p, p^{\prime}\right)$ (a qual é um ponto ou um segmento), e então $\Omega(q)$ não pode conter um top-ciclo.

Observação 5.2 Se fé suave, então quaisquer dois top-ciclos devem ser disjuntos pois a normal a $f(M)$ em um ponto em $\Omega(p)$ deve ser $p$. Entretanto, se $f$ é assumida só contínua, então dois top-ciclos podem se interceptar em um ponto ou um segmento de reta.

O resultado a seguir decompõe a variedade $M$ na união disjunta de dois abertos não-vazios $U$ e $V$ cuja fronteira comum é a união dos top-ciclos, em número finito, $\gamma_{1}, \ldots, \gamma_{k}$. Além disso, se $f$ é assumida diferenciável, então a curvatura Gaussiana $K$ é tal que $K \geq 0$ em $U$ e $K \leq 0$ em $V$.

Teorema 5.1 Seja $f: M \rightarrow \mathbf{E}^{3}$ uma imersão topológica justa de uma superfície compacta e conexa $M$ não homeomorfa à esfera $S^{2}$. Então $M$ é a união de dois abertos disjuntos não-vazios $U$ e $V$ e dos top-ciclos (finitos) $\gamma_{1}, \ldots, \gamma_{k}$ satisfazendo: 
(a) $O$ conjunto $U$ '́ mergulhado sobre o complementar em $\partial \mathcal{H} f(M)$ de um número finito de discos convexos fechados planos $D_{1}, \ldots, D_{k}$, onde $\gamma_{i}=f^{-1}\left(\partial D_{i}\right)$, $1 \leq i \leq k$.

(b) Se $f$ é suave, então a curvatura Gaussiana $K$ é tal que $K \geq 0$ em $U e$ $K \leq 0 \mathrm{em} V$.

\section{Demonstração:}

(a) Deve existir algum top-ciclo, pois caso contrário, $f$ mergulha $M$ sobre $\partial \mathcal{H} f(M)$, e $M$ é uma esfera. Sejam $\gamma_{1}, \ldots, \gamma_{k}$ os top-ciclos de $f$ e $D_{1}, \ldots, D_{k}$ os discos convexos fechados planos tais que $f\left(\gamma_{i}\right)=\partial D_{i}, 1 \leq i \leq k$. Já sabemos que $\partial \mathcal{H} f(M)=\bigcup_{\Omega} \mathcal{H} f(\Omega)$. Seja $W=\partial \mathcal{H} f(M)-\left(D_{1} \cup \cdots \cup D_{k}\right)$. Se $p \in W$, então pelo lema $4.4, p \in f(\Omega)=\mathcal{H} f(\Omega)$, onde $\Omega$ é um top-set tal que $\mathcal{H} f(\Omega)$ tem dimensão 0 ou 1 , ou tal que $\mathcal{H} f(\Omega)$ é 2 -dimensional e, neste caso, $f$ mergulha $\Omega$ sobre $\mathcal{H} f(\Omega)$. Logo, $f$ é um mergulho em $U=f^{-1} W$. Pela parte (b) do lema $4.4, f$ é ainda um mergulho no conjunto fechado

$$
A=U \cup\left(\gamma_{1} \cup \cdots \cup \gamma_{k}\right)
$$

Seja $V=M-A$. Então,

$$
M=U \cup V \cup\left(\gamma_{1} \cup \cdots \cup \gamma_{k}\right)
$$

(b) O lema de Morse (1.1) e o teorema 1.9 mostram que uma imersão diferenciável tem um plano suporte local estrito em pontos onde $K>0$, e não tem plano suporte local onde $K<0$. Se $x \in U$, então $f$ tem um plano suporte local em $f(x)$ pois $f(x) \in \partial \mathcal{H} f(M)$, e portanto $K \geq 0$. Por outro lado,suponha que $K(x)>0$ em um ponto $x \in V$. Então, se $p$ é o vetor unitário normal ao plano tangente a $f(x)$, podemos supor que $\ell_{p}$ tem um máximo não degenerado em $x$. Pelo corolário 1.4, existe uma função altura não degenerada $\ell_{q}$ que tem um máximo local próximo de $x$ e portanto em $V$. Mas, $\ell_{q}$ assume seu máximo global em $\partial \mathcal{H} f(M)$ e portanto em um ponto $y \in U$. Logo, $\ell_{q}$ não é polar, contrariando a justeza.

Procederemos agora para mostrar que o Plano Projetivo e a a Garrafa de Klein não admitem imersões justas em $\mathbf{E}^{3}$, nem mesmo topológicas. No caso do plano projetivo, Kuiper [14] mostrou que se existir uma tal imersão então esta admite no máximo um top-ciclo. Em 1984 Cecyl e Ryan [7] estabeleceram limites para o número de top-ciclos (o qual é finito) de imersões topológicas justas. Apresentamos a seguir este resultado. 
Teorema 5.2 Seja $f: M \rightarrow \mathbf{E}^{3}$ uma imersão topológica justa de uma superfície compacta e conexa não homeomorfa a $S^{2}$ e, seja $\alpha(f)$ o número de top-ciclos de f. Então

$$
2 \leq \alpha(f) \leq 2-\chi(M) .
$$

Além disso, se $\alpha(f)=2-\chi(M)$, então os top-ciclos aparecem aos pares, ligados por um cilindro topológico.

\section{Demonstração:}

Como $M$ não é $S^{2}$ deve existir algum top-ciclo. Não pode existir apenas um top-ciclo, pois pelo teorema 5.1, este top-ciclo separaria $M$, contrariando a definição de top-ciclo. Então $\alpha(f) \geq 2$. Sejam $U$ e $V$ como no teorema 5.1 e suponhamos inicialmente que todos os top-ciclos sejam disjuntos. Sejam $V_{1}, \cdots, V_{k}$ as componentes conexas de $V$. Cada $\bar{V}_{i}$ é uma variedade com fronteira consistindo de $s_{i}$ top-ciclos onde $s_{i} \geq 2$, já que um único top-ciclo não separa. Então $\chi\left(\overline{V_{i}}\right) \leq 0$. De fato, a superfície $V_{i}^{*}$ obtida de $\overline{V_{i}}$ adicionando-se a cada componente de bordo um disco convexo é uma superfície compacta sem bordo e

$$
\chi\left(V_{i}^{*}\right)=\chi\left(\overline{V_{i}}\right)+s_{i} .
$$

Por outro lado, pelo teorema de classificação de superfícies [20], temos que $\chi\left(V_{i}^{*}\right) \leq$ 2. Portanto, $\chi\left(\overline{V_{i}}\right) \leq 0$, e além disso, $\chi\left(\overline{V_{i}}\right)=0$ se, e somente se, $V_{i}^{*}$ é uma esfera, isto é, se $s_{i}=2$.

Seja $A$ a união de $U$ e dos top-ciclos. Lembremos que

$$
U \sim \partial \mathcal{H} f(M)-\left(D_{1} \cup \cdots \cup D_{\alpha(f)}\right),
$$

onde $\gamma_{i}=f^{-1}\left(\partial D_{i}\right)$. Mas, $\partial \mathcal{H} f(M) \sim S^{2}$ e, então

$$
\begin{aligned}
A & =U \cup\left(\gamma_{1} \cup \cdots \cup \gamma_{\alpha(f)}\right) \\
& \sim \partial \mathcal{H} f(M)-\left(D_{1} \cup \cdots \cup D_{\alpha(f)}\right) \cup \bigcup_{i=1}^{\alpha(f)} \gamma_{i} \\
& \sim S^{2}-\left(\operatorname{int} D_{1} \cup \cdots \cup \operatorname{int} D_{\alpha(f)}\right),
\end{aligned}
$$

onde $D_{i} \cap D_{j}=\emptyset$ se $i \neq j$. Portanto, $\chi(A)=\chi\left(S^{2}\right)-\alpha(f)=2-\alpha(f)$. Como

$$
M=A \cup \overline{V_{1}} \cup \cdots \cup \overline{V_{k}},
$$

e $A$ e $\bar{V}_{i}$ se interceptam em uma união disjunta de curvas, então

$$
\chi(M)=\chi(A)+\chi\left(\overline{V_{1}}\right)+\cdots+\chi\left(\overline{V_{k}}\right) .
$$


Logo,

$$
\chi(M) \leq 2-\alpha(f),
$$

e daí

$$
\alpha(f) \leq 2-\chi(M) .
$$

A igualdade se verifica se, e somente se, $\chi\left(\overline{V_{i}}\right)=0 \forall i$, isto é, se e somente se, $V_{i}$ é um cilindro.

Suponhamos agora que os top-ciclos não sejam disjuntos. Como $f$ é um mergulho em uma vizinhança de $A$, podemos substituir $A$ por um conjunto maior $Z$ cuja fronteira consiste de $\alpha(f)$ curvas disjuntas próximas aos top-ciclos. Então repetimos o argumento acima substituindo os $V_{i}$ pelas componentes de $M-Z$. Ainda neste caso, se $\alpha(f)=2-\chi(M)$, então cada componente $V_{i}$ de $V$ é um cilindro aberto.

Lembrando que $\chi\left(\mathbf{P}^{2}\right)=1$ e aplicando o teorema 5.2 acima concluimos que

Teorema 5.3 Não existe imersão topológica justa do plano projetivo $\mathbf{P}^{2}$ em $\mathbf{E}^{3}$.

O próximo teorema no qual mostramos a não existência de imersão justa da Garrafa de Klein em $\mathbf{E}^{3}$ é o resultado mais difícil desta seção. A idéia da demonstração é a seguinte: começamos com uma imersão justa $f$ definida em uma superfície compacta sem bordo com caracteristica de Euler igual a zero, isto é, uma Garrafa de Klein ou um Toro de revolução. $\mathrm{O}$ objetivo é mostrar que esta superfície é orientável e, portanto não pode ser a Garrafa de Klein. Faremos a prova somente para o caso diferenciável, mas o resultado topológico também se verifica.

Teorema 5.4 Não existe imersão justa da Garrafa de Klein em $\mathbf{E}^{3}$.

\section{Demonstração:}

Seja $M$ uma superfície compacta com característica de Euler $\chi(M)=0$, isto é, $M$ é o toro de revolução ou a garrafa de Klein. Mostraremos que se existir uma imersão justa $f: M \rightarrow \mathbf{E}^{3}$ então $M$ deve ser orientável. Suponhamos então que exista tal imersão. Pelo teorema 5.2, como $\chi(M)=0$, devem existir exatamente dois top-ciclos $\gamma_{1}$ e $\gamma_{2}$ e os mesmos são conectados por um cilindro $V$ de curvatura não positiva. Note que $\gamma_{1}$ e $\gamma_{2}$ são disjuntos desde que $f$ é diferenciável. Considerando uma transfomação projetiva podemos assumir que 
$f\left(\gamma_{1}\right)$ e $f\left(\gamma_{2}\right)$ estão em planos paralelos, digamos $\ell_{p}=r_{1}$ e $\ell_{p}=r_{2}$ com $r_{1}>r_{2}$. $\mathrm{O}$ top-set $\Omega(p)$ é conexo e, então podemos escolher um campo de vetores normais unitários de $\Omega(p)$, a saber, o campo constante $\{p\}$. Podemos estender esta escolha de vetores normais para produzir uma vizinhança orientada de $\Omega(p)$ em $M$ a qual tem uma aplicação de Gauss bem definida em $S^{2}$. Seja $N_{1}$ uma vizinhança de $p$ em $S^{2}$ pequena o bastante para que dois vetores quaisquer nunca sejam perpendiculares, e $U_{1}$ a imagem inversa de $N_{1}$ pela aplicação de Gauss. $U_{1}$ é, então uma vizinhança de $\Omega(p)$. Escolha uma vizinhança $U_{2}$ de $\Omega(-p)$ do mesmo modo. Pelo lema chave (lema 4.1), existe uma $\ell_{q}$ não degenerada com $q$ próximo de $p$ tal que

$$
\Omega(p) \subset \bar{M}_{b}^{+}(q) \subset U_{1} \quad \Omega(-p) \subset \bar{M}_{a}^{-}(q) \subset U_{2}
$$

para valores não críticos apropriados $a<b$. As variedades compactas com fronteira $\bar{M}_{b}^{+}(q)$ e $\bar{M}_{a}^{-}(q)$ contém os top-ciclos $\gamma_{1}$ e $\gamma_{2}$, respectivamente. Logo, nenhuma delas é homotópica a um ponto. Como $\ell_{q}$ tem 4 pontos críticos em $M$, ela deve ter um máximo e uma sela em $\bar{M}_{b}^{+}(q)$ e um mínimo e uma sela em $\bar{M}_{a}^{-}(q)$. Então, estas variedades têm característica de Euler zero e, como são orientáveis, elas são cilindros.

Sejam $U$ e $V$ como no teorema 5.1. Existem $x, y \in U$ tais que $\ell_{q}(x)<a \leq$ $s \leq b<\ell_{q}(y)$ e, portanto pelo Teorema do Valor Intermediário, existe $z \in U$ tal que $\ell_{q}(z)=s$. O mesmo é válido para $V$. Logo, o plano $\ell_{q}=s$ intercepta $f(U)$ e $f(V), \forall s \in[a, b]$. Como não existem valores críticos de $\ell_{q}$ em $[a, b]$, pelo teorema 1.1 segue que $\bar{M}_{s}^{-}(q)$ é um retrato por deformação de $\bar{M}_{a}^{-}(q)$ e, portanto também é um cilindro cuja fronteira consiste de duas curvas suaves $\alpha_{s}$ em $U$ e $\beta_{s}$ em $V$.

$\mathrm{O}$ conjunto $U \cup \bar{M}_{b}^{+}(q) \cup \bar{M}_{a}^{-}(q)$ é um cilindro topológico. Coloquemos uma orientação em uma vizinhança $W$ deste cilindro e nos planos paralelos $\ell_{q}=s$, $a \leq s \leq b$. Esta orientação induz uma orientação em cada uma das curvas planas diferenciáveis $\alpha_{s}$. Identificando os planos paralelos, temos uma família diferenciável $\left\{\alpha_{s}\right\}$ de curvas diferenciávies mergulhadas, todas das quais devem ter o mesmo índice de rotação, digamos +1 . Esta orientação de $W$ induz também uma orientação nas curvas $\beta_{b}$ e $\beta_{a}$. A curva orientada $\beta_{b}$ é inersa no mesmo plano que $\alpha_{b}$. Mostremos que estas duas curvas têm o mesmo índice de rotação. As curvas $\alpha_{b}$ e $\beta_{b}$ formam a fronteira do cilindro orientado $\bar{M}_{b}^{+}(q)$ e então existe uma homotopia diferenciável de curvas mergulhadas $\phi_{t}, 0 \leq t \leq 1$, em $\bar{M}_{b}^{+}(q)$ satisfazendo $\phi_{0}=\alpha_{b}$ e $\phi_{1}=\beta_{b}$. Relembrando a construção de $U_{1}$, temos que $q \in N_{1}$, já que $\ell_{q}$ tem um ponto crítico em $U_{1}$. Pela definiçâo de $N_{1}, q$ não é tangente a $M$ em nenhum ponto de $U_{1}$. Portanto, se $\pi$ é a projeção ortogonal de $\mathbf{E}^{3}$ sobre o plano $\ell_{q}=b$, então $\pi$ o $f$ é uma imersão em $U_{1}$. Logo, a família de curvas $\pi \circ f\left(\phi_{t}\right)$ é uma homotopia regular de curvas regulares no plano $\ell_{q}=b$ e as 
curvas $\pi \circ f\left(\phi_{0}\right)=f\left(\alpha_{b}\right)$ e $\pi \circ f\left(\phi_{1}\right)=f\left(\beta_{b}\right)$ tem o mesmo índice de rotação +1 . Com um argumento análogo mostramos que $f\left(\alpha_{a}\right)$ e $f\left(\beta_{b}\right)$ têm o mesmo índice de rotação +1 , no plano $\ell_{q}=a$.

Seja $C$ o cilindro que é a união das curvas $\beta_{a}, a \leq s \leq b$. Uma escolha de orientação para $C$ torna $\left\{\beta_{s}\right\}$ uma família diferenciável de curvas planas orientadas (ainda identificando os planos paralelos), que devem ter portanto, o mesmo índice de rotação. Vimos acima que o índice de rotação de $\beta_{b}$ deve ser +1 ou -1 . Escolhemos então a orientação para $C$ que dá índice de rotação +1 para todas as $\beta_{s}$. Então $\beta_{a}$ e $\beta_{b}$ tem mesma orientação que aquela induzida de $W$. Assim, temos uma orientação consistente para $M$, que é portanto um toro e não uma garrafa de Klein.

Usando a versão topológica do teorema 5.4 podemos obter um limite melhor para o número de top-ciclos de uma imersão justa de uma superfície não-orientável em $\mathbf{E}^{3}$. Assumiremos novamente que os top-ciclos são disjuntos.

Teorema 5.5 Seja $f: M \rightarrow \mathbf{E}^{3}$ uma imersão topológica justa de uma superfície conexa compacta e náo-orientável e, seja $\alpha(f)$ o número de top-ciclos. Então $2 \leq \alpha(f) \leq 1-\chi(M)$.

\section{Demonstração:}

Mostramos no teorema 5.2 que $2 \leq \alpha(f) \leq 2-\chi(M)$. Se a igualdade se verifica, então $\alpha(f)$ é duas vezes o número de componentes de $V$ (usando a decomposição de $M$ no teorema 5.1)e, então $\chi(M)$ é par. Assim, se $\chi(M)$ é impar temos $\alpha(f)<2-\chi(M)$ e, portanto $\alpha(f) \leq 1-\chi(M)$. Suponhamos então que $\chi(M)$ é par e que $\alpha(f)=2-\chi(M)$. Então, pelo teorema 5.2 existem $K=\frac{\alpha(f)}{2}$ cilindros $V_{i}$ ligados aos correspondentes pares de top-ciclos. Seja $A$ a união de $U$ com os top-ciclos. Podemos colocar uma orientação em $f(A)$ a qual induz uma orientação em cada uma das curvas convexas que são imagens dos top-ciclos. Podemos também orientar cada cilindro $V_{i}$, induzindo portanto, uma orientação nos top-ciclos. Para pelo menos um destes cilindros, digamos $V_{1}$, nenhuma orientação possível de $V_{1}$ é consistente com a orientação de $A$ em ambas as curvas da fronteia, pois caso contrário, $M$ seria orientável. Vamos substituir os cilindros $V_{2}, \ldots, V_{k}$ pelos discos convexos limitados pelos seus top-ciclos. A nova superfície assim obtida ainda tem a PDP. Então, ela é uma imersão contínua e justa da garrafa de Ḱlein, contrariando a versão topológica do teorema 5.4. Portanto, $\alpha(f)<2-\chi(M)$ e concluimos que $\alpha(f) \leq 1-\chi(M)$. 


\subsection{Imersões Justas de Superfícies com Carac- terística de Euler ímpar em $\mathbf{E}^{3}$}

Nesta seção apresentaremos, sem entrar em detalhes, os resultados sobre existência ou não de imersões justas de superfícies com característica de Euler ímpar $\chi \leq-1$.

Kuiper construiu exemplos de imersões justas de todas as superfícies com característica de Euler ímpar $\chi \leq-3$. A construção principal e também a mais complicada ([15]) é o caso $\chi=-3$, ou seja, o plano projetivo com duas alças $M=\mathbf{P}^{2} \# T^{2} \# T^{2}$. Devido a complexidade desta construção não a apresentaremos aqui. O passo principal é a construção de uma imersão de $\mathbf{P}^{2} \# T^{2}$ não justa, obtida por Kuiper, na tentativa de obter uma imersão justa desta superfície. Em seguida, ele adiciona uma alça de curvatutra negativa retirando um pequeno disco de curvatura positiva da referida imersão, obtendo assim, uma imersão justa de $\mathbf{P}^{2} \# T^{2} \# T^{2}$ em $\mathbf{E}^{3}$. Os passos desta construção são descritos por Cecil e Ryan em [6]. Para obtermos uma imersão justa de $M \operatorname{com} \chi(M) \leq-5$ impar, adicionamos um número conveniente de alças à imersão justa de $\mathbf{P}^{2} \# T^{2} \# T^{2}$.

O problema de existência de imersão justa do plano projetivo com uma alça $M=\mathbf{P}^{2} \# T^{2}, \chi(M)=-1$, permaneceu em aberto por cerca de 30 anos, provocando o aparecimento de resultados equivalentes à uma resposta positiva deste problema. Por exemplo citamos o seguinte teorma de Cecil e Ryan

Teorema 5.6 ([7]) Seja $M$ uma superfície conexa e compacta com característica de Euler ímpar $\chi(M)<-1$. Então existe uma imersão topológica (diferenciável) justa $f: M \rightarrow \mathbf{E}^{3}$ com $\alpha(f)=1-\chi(M)$ se, e somente se, existe uma imersão topológica (diferenciável) justa de $\mathbf{P}^{2} \# T^{2}$ em $\mathbf{E}^{3}$.

Recentemente, F. Haab ([11]) mostrou que $M=\mathbf{P}^{2} \# T^{2}$ não admite imersões justas em $\mathbf{E}^{3}$. Para a demonstração deste resultado, Haab faz um estudo das imersões justas e das aplicações estáveis justas de superfícies no plano, encontrando obstruções para a existência de uma fatoração de uma aplicação justa estável $F: M \rightarrow \mathbf{E}^{2}$ por imersões justas $f: M \rightarrow \mathbf{E}^{3}$, seguidas de projeções ortogonais.

Teorema 5.7 (F. Haab,[11]) Não existe imersão justa do plano projetivo com uma alça em $\mathbf{E}^{3}$. 


\subsection{Aplicações $C^{\infty}$-Estáveis Justas}

Apesar de não existirem imersões justas do plano projetivo $\mathbf{P}^{\mathbf{2}}$, da garrafa de Klein $\mathbf{K}^{2}$ e do plano projetivo com uma alça $\mathbf{P}^{2} \# T^{2}$ em $\mathbf{E}^{3}$, Kuiper verifica em [16] a existência de aplicações $C^{\infty}$-estáveis justas de todas as superfícies em $\mathbf{E}^{3}$. Apresentaremos nesta secão uma família

$$
g_{t}: \mathbf{P}^{2} \rightarrow \mathbf{E}^{3}
$$

a um parâmetro de aplicações justas do plano projetivo em $\mathbf{E}^{3}$. Esta família, estudada por Banchoff em [3] apresenta singularidades $C^{\infty}$-estáveis para todo valor do parâmetro exceto por um único ponto $t=t_{0}$ de bifurcação. Para $t<t_{0}$ as imagens $g_{t}\left(\mathbf{P}^{2}\right)$ são as supefícies singulares denominadas Cross-Caps de Steiner que apresentam 2 cross-caps. Para $t>t_{0}$ as imagens $g_{t}\left(\mathbf{P}^{2}\right)$ são as superfícies singulares denominadas Superficies Romanas de Steiner as quais apresentam 6 cross-caps. $O$ valor de transição $t=t_{0}$ corresponde ao cancelamento simultâneo de tipo hiperbólico de 2 pares de cross-caps.

Faremos, no que segue, uma análise detalhada da família descrita acima, seguindo as idéias apresentadas por Apéry em [1].

Definição 5.2 Uma aplicação $f: M \rightarrow \mathbf{E}^{m}$ de uma variedade diferenciável é dita $C^{\infty}$-estável se para qualquer $g$ suficientemente próxima de $f$ na topologia $C^{\infty}$ de Whitney existem difeomorfismos $\varphi: M \rightarrow M$ e $\psi: \mathbf{E}^{m} \rightarrow \mathbf{E}^{m}$ tais que

$$
g=\psi \circ f \circ \varphi^{-1} \text {. }
$$

H. Whitney mostrou em 1944 ([WH2] de [1], pag. 62) que a única singularidade estável de aplicações de $\mathbf{R}^{2}$ em $\mathbf{R}^{3}$ é o cross-cap ou guarda-chuva de Whitney, que em um sistema de coordenadas apropriado na origem, pode ser escrita como

$$
(x, y) \rightarrow\left(x, x y, y^{2}\right)
$$

Consideremos o mergulho (justo) de Veronese do plano projetivo $\mathbf{P}^{2}$ em $\mathbf{E}^{4}$ dado por

$$
f:(x, y, z) \rightarrow\left(\frac{x^{2}-y^{2}}{\sqrt{2}}, \sqrt{2} x z, \sqrt{2} x y, \sqrt{2} y z\right) .
$$

Vamos estudar o comportamento de uma família de projeções desta superfície em subespaços tridimensionais. Começamos analisando a aplicação obtida projetandose $f$ em suas três primeiras coordenadas, isto é, a aplicação

$$
g_{0}:(x, y, z) \rightarrow\left(\frac{x^{2}-y^{2}}{\sqrt{2}}, \sqrt{2} z x, \sqrt{2} x y\right) .
$$


Mostremos que $g_{0}$ tem dois guarda-chuvas de Whitney.

Parametrizemos a esfera $S^{2}$ por $z=\sqrt{1-x^{2}-y^{2}}$. Entào,

$$
g_{0}(x, y)=\left(\frac{x^{2}-y^{2}}{\sqrt{2}}, \sqrt{2} x \sqrt{1-x^{2}-y^{2}}, \sqrt{2} x y\right) .
$$

Sua matriz jacobiana é dada por

$$
\left(\begin{array}{cc}
\frac{2 x}{\sqrt{2}} & \frac{-2 y}{\sqrt{2}} \\
\frac{\sqrt{2}\left(1-2 x^{2}-y^{2}\right)}{\sqrt{1-x^{2}-y^{2}}} & \frac{-\sqrt{2} x y}{\sqrt{1-x^{2}-y^{2}}} \\
\sqrt{2} y & \sqrt{2} x
\end{array}\right)
$$

É fácil ver que a matriz acima tem posto menor que 2 se, e somente se $x=$ $y=0$. Portanto $(0,0)$ é ponto singular de $g_{0}(x, y)$, isto é, $[(0,0,1)] \in \mathbf{P}^{2}$ é ponto singular da aplicação $g_{0}$. Mostremos que este ponto é um guarda-chuva de Whitney. Indiquemos por $(u, v, t)$ as coordenadas da meta. Então,

$$
g_{0}(x, y)=\left\{\begin{aligned}
u & =\frac{x^{2}-y^{2}}{\sqrt{2}} \\
v & =\sqrt{2} \sqrt{1-x^{2}-y^{2}} x \\
t & =\sqrt{2} x y
\end{aligned}\right.
$$

Façamos a seguinte mudança de coordenadas na fonte

$$
\begin{aligned}
& X=v \\
& Y=y
\end{aligned}
$$

Como $\sqrt{1-x^{2}-y^{2}} \neq 0$ em uma vizinhança de $(0,0)$ a aplicação acima é difeomorfismo $C^{\infty}$ nesta vizinhança e sua inversa é da forma

$$
\begin{aligned}
& x=X+\cdots \\
& y=Y+\cdots
\end{aligned}
$$

Assim, temos:

$$
\begin{aligned}
u & =\frac{X^{2}-Y^{2}}{\sqrt{2}}+\cdots \\
v & =X \\
t & =\sqrt{2} X Y+\cdots
\end{aligned}
$$


Fazendo então a seguinte mudança na fonte

$$
\begin{aligned}
U & =-u+v^{2} \\
V & =v \\
T & =t
\end{aligned}
$$

obtemos

$$
\begin{aligned}
& U=Y^{2} \\
& V=X \\
& T=X Y
\end{aligned}
$$

ou seja, em uma vizinhança de $(0,0) g_{0}$ é um cross-cap.

Por outro lado, considerando a parametrização $y=\sqrt{1-x^{2}-z^{2}}$ a matriz jacobiana de $g_{0}$ será dada por

$$
\left(\begin{array}{cc}
\frac{-4 x}{\sqrt{2}} & \frac{2 z}{\sqrt{2}} \\
\sqrt{2} z & \sqrt{2} x \\
\sqrt{2} \frac{1-2 x^{2}-z^{2}}{\sqrt{1-x^{2}-z^{2}}} & \frac{-\sqrt{2} x z}{\sqrt{1-x^{2}-z^{2}}}
\end{array}\right)
$$

Novamente, a matriz acima tem posto menor que 2 , dentro do domínio da parametrização se, e somente se, $x=z=0$. Portanto, o ponto $[(0,1,0)] \in \mathbf{P}^{2}$ é uma singularidade de $g_{0}$. Como antes, mostra-se que $[(0,1,0)]$ é um guarda-chuva de Whitney.

Em seguida, rodando no plano da primeira e quarta variáveis a projeção nas três primeiras coordenadas dá agora a superfície Romana de Steiner

$$
g_{\frac{\pi}{2}}(x, y, z)=(\sqrt{2} y z, \sqrt{2} x z, \sqrt{2} x y),
$$

a qual mostraremos ter exatamente seis pinch-points. Novamente, começamos com a parametrização

$$
\begin{gathered}
z=\sqrt{1-x^{2}-y^{2}}, \\
g_{\frac{\pi}{2}}(x, y)=\left(\sqrt{2} y \sqrt{1-x^{2}-y^{2}}, \sqrt{2} x \sqrt{1-x^{2}-y^{2}}, \sqrt{2} x y\right) .
\end{gathered}
$$

A jacobiana de $g_{\frac{\pi}{2}}$, neste caso, é dada por

$$
\left(\begin{array}{cc}
-\frac{\sqrt{2} x y}{\sqrt{1-x^{2}-y^{2}}} & \sqrt{2} \frac{1-x^{2}-2 y^{2}}{\sqrt{1-x^{2}-y^{2}}} \\
\sqrt{2} \frac{1-2 x^{2}-y^{2}}{\sqrt{1-x^{2}-y^{2}}} & -\frac{\sqrt{2} x y}{\sqrt{1-x^{2}-y^{2}}} \\
\sqrt{2} y & \sqrt{2} x
\end{array}\right)
$$




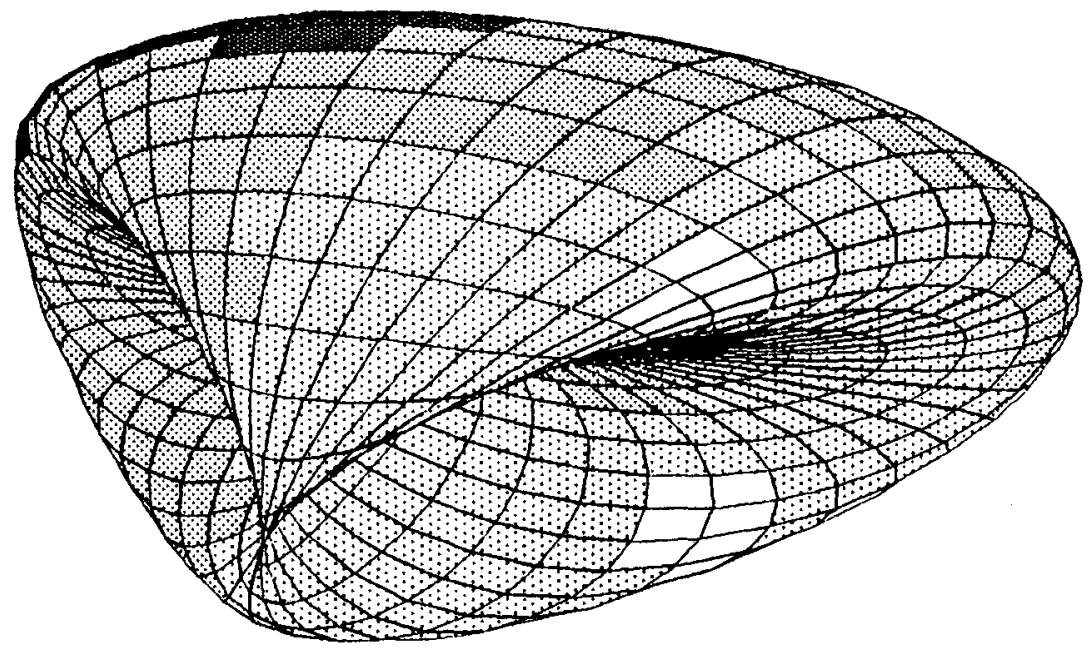

Figura 5.5: Cross-cap de Steiner

cujos menores são:

$$
\begin{gathered}
\frac{2 x\left(1-2 x^{2}\right)}{\sqrt{1-x^{2}-y^{2}}} \\
-\frac{2 y\left(1-2 y^{2}\right)}{\sqrt{1-x^{2}-y^{2}}} \\
1-3 x^{2}-3 y^{2}+2 x^{4}+2 y^{4}+4 x^{2} y^{2} .
\end{gathered}
$$

Destas equações segue que os seis pontos

$$
\left(0, \pm \frac{\sqrt{2}}{2}\right),\left( \pm \frac{\sqrt{2}}{2}, 0\right),\left(\frac{\sqrt{2}}{2}, \pm \frac{\sqrt{2}}{2}\right)
$$

são as singularidades de $g_{\frac{\pi}{2}}$. Estes pontos são guarda-chuvas de Whitney. Por exemplo, o 2-jato de $g_{\frac{\pi}{2}}$ em uma vizinhança do ponto $\left(0, \frac{\sqrt{2}}{2}\right)$ tem a seguinte forma normal

$$
\left(-2 \sqrt{2} x^{2}-\frac{\sqrt{2}}{2} y^{2}, y, x y\right)
$$

e portanto, o ponto $\left[\left(0, \frac{\sqrt{2}}{2}, \frac{\sqrt{2}}{2}\right)\right] \in \mathbf{P}^{2}$ é um pinch-point. Formas normais análogas se verificam para os demais pontos.

Observemos que ao rodarmos de $g_{0}$ para $g_{\frac{\pi}{2}}$ devemos passar por posições intermediárias de rotação que são representadas pela família

$$
g_{t}: \mathbf{P}^{2} \rightarrow \mathbf{E}^{3}, 0 \leq t \leq \frac{\pi}{2}
$$




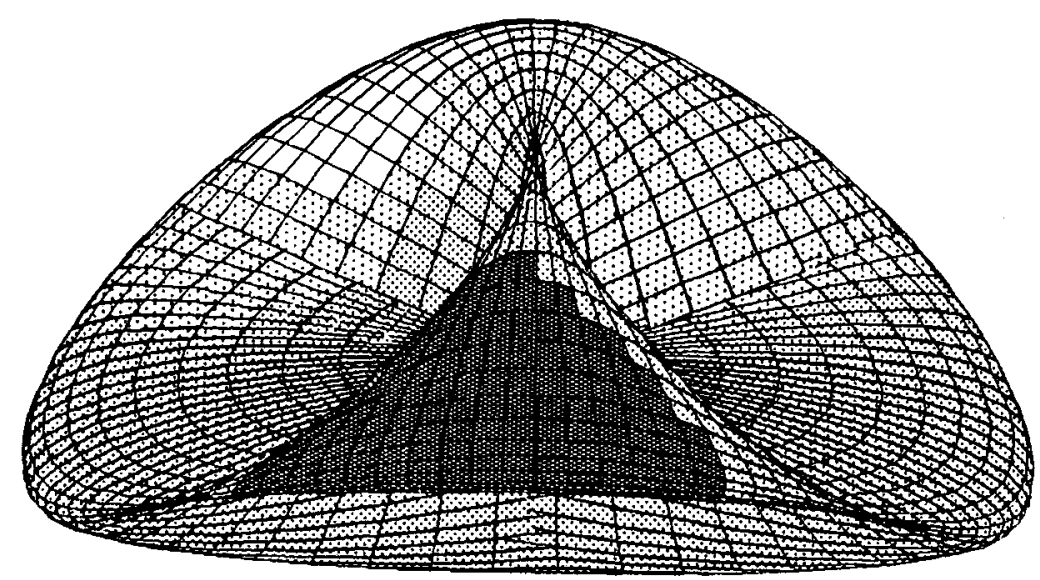

Figura 5.6: Superficie Romana de Steiner

onde

$$
g_{t}(x, y, z)=\left(\cos (t) \frac{x^{2}-y^{2}}{\sqrt{2}}+\operatorname{sen}(t) \sqrt{2} y z, \sqrt{2} x z, \sqrt{2} x y\right)
$$

É esta família que passamos a estudar.

Como anteriormente, tomemos a parametrização $g_{t}:(x, y) \rightarrow\left(\cos (t) \frac{x^{2}-y^{2}}{\sqrt{2}}+\operatorname{sen}(t) \sqrt{2} y \sqrt{1-x^{2}-y^{2}}, \sqrt{2} x \sqrt{1-x^{2}-y^{2}}, \sqrt{2} x y\right)$ cuja matriz jacobiana tem os seguintes menores

$$
\begin{aligned}
J\left(f_{2}, f_{3}\right)= & \frac{2 x\left(1-2 x^{2}\right)}{\sqrt{1-x^{2}-y^{2}}} \\
J\left(f_{1}, f_{3}\right)= & 2 \cos (t)\left(x^{2}+y^{2}\right)-\frac{2 \operatorname{sen}(t) y}{\sqrt{1-x^{2}-y^{2}}}\left(1-2 y^{2}\right) \\
J\left(f_{1}, f_{2}\right)= & \frac{2 \cos (t) y}{\sqrt{1-x^{2}-y^{2}}}\left(1-3 x^{2}-y^{2}\right)+ \\
& \frac{2 \operatorname{sen}(t)}{1-x^{2}-y^{2}}\left(x^{2} y^{2}-\left(1-x^{2}-2 y^{2}\right)\left(1-2 x^{2}-y^{2}\right)\right)
\end{aligned}
$$

onde $f_{1}, f_{2}, f_{3}$ são as funções coordenadas de $g_{t}$

Note que $J\left(f_{2}, f_{3}\right)=0$ se, e somente se, $x=0$ ou $x= \pm \frac{\sqrt{2}}{2}$. Substituindo 


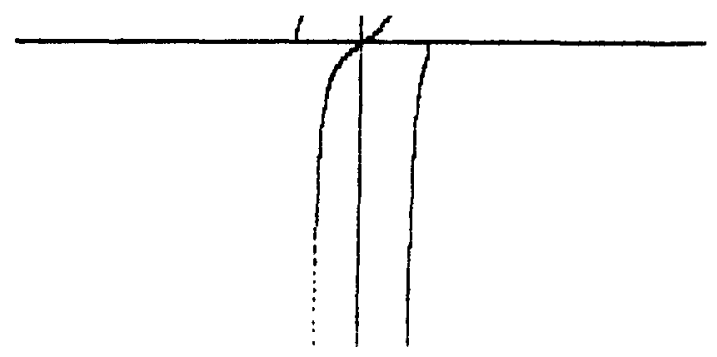

Figura 5.7: Gráfico de $t_{1}(y)$

$x=0$ em $J\left(f_{1}, f_{3}\right)$ e igualando a zero obtemos

$$
t(y)=\arctan \left(\frac{y \sqrt{\left(1-y^{2}\right)}}{1-2 y^{2}}\right)
$$

enquanto que para $x= \pm \frac{\sqrt{2}}{2}$ a equação obtida é

$$
t(y)=\arctan \left(\frac{\left(1+2 y^{2}\right) \sqrt{1 / 2-y^{2}}}{y\left(2-4 y^{2}\right)}\right)
$$

Uma análise da função 5.5 (figura 5.7) mostra que para cada valor de $t$ correspondem dois valores de $y$, digamos, $y_{1}(t), y_{2}(t)$. Por outro lado, é fácil ver que a função 5.6 tem uma tangência horizontal no ponto $\left(-\frac{\sqrt{6}}{6}, \arctan (\sqrt{2})\right)$ (figura 5.8). Analisando, esta função vemos que para cada valor do parâmetro $t>\arctan (\sqrt{2})$ existem novamente dois valores de $y$ correspondentes, $y_{3}(t), y_{4}(t)$, enquanto que $t=\arctan (\sqrt{2})$ é obtido somente para $y=\frac{\sqrt{6}}{6}$. A proposição a seguir resume esta discussão

Proposição 5.1 A familia $g_{t}$ é tal que

$$
\left\{\begin{array}{lll}
g_{t} \text { tem seis pinch-points se } & t>\arctan (\sqrt{2}) \\
g_{t} \text { tem dois pinch-points se } & t<\arctan (\sqrt{2}) \\
g_{t} \quad \text { tem dois pinch-points } e & \\
& \text { duas singularidades mais degeneradas se } & t=\arctan (\sqrt{2})
\end{array}\right.
$$

Para analisar a passagem de 6 pinch-points para 2 pinch-points na família $g_{t}$, seguimos o procedimento indicado por Apéry em [1]. Mostraremos que no valor do parâmetro $t=\arctan (\sqrt{2})$, ocorre confluência de guarda-chuvas, que passamos a definir. 


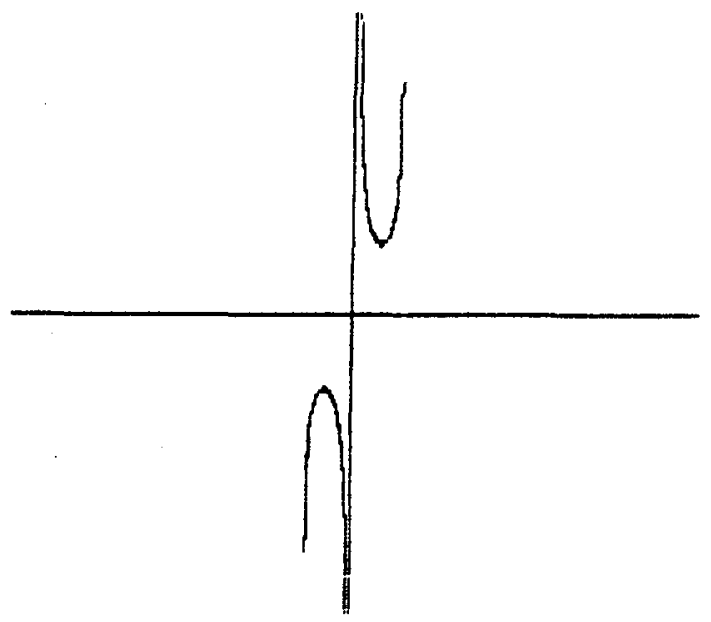

Figura 5.8: Gráfico de $t_{2}(y)$

Definição 5.3 Uma confluência de guarda-chuvas de Whitney é qualquer deformação $f_{t}$ induzida por um germe $F:\left(\mathbf{R}^{3}, 0\right) \rightarrow\left(\mathbf{R}^{4}, 0\right)$ do tipo

$$
F(t, x, y)=(t, \bar{f}(t, x, y))
$$

onde $f_{t}(x, y)=\bar{f}(t, x, y)$ tal que a curva de guarda-chuvas de Whitney do germe $F$ é regular na origem e tangente ao plano $t=0 \mathrm{com}$ contato de ordem dois. Dizemos que a confluência é elíptica se em uma vizinhança da origem a deformação é dada por

$$
f_{t}(x, y)=\left(x, y^{2}, x^{2} y+y^{3}-t y\right) .
$$

Se $f_{t}$ tem a seguinte forma local em uma vizinhança da origem

$$
f_{t}(x, y)=\left(x, y^{2}, x^{2} y-y^{3}-t y\right)
$$

então dizemos que a confluência é hiperbólica.

Observação 5.3 Existem somente estes dois tipos de confluência de guardachuvas de Whitney. Este resultado é devido a Wallace Collao e é citado por Apéry ([1]).

Consideremos o germe de $F: \mathbf{R}^{3} \rightarrow \mathbf{R}^{4}$ que tem as seguintes funções coordenadas

$$
\begin{aligned}
& F_{0}(t, x, y)=t \\
& F_{1}(t, x, y)=\cos (t) \frac{x^{2}-y^{2}}{\sqrt{2}}+\operatorname{sen}(t) \sqrt{2} y \sqrt{1-x^{2}-y^{2}} \\
& F_{2}(t, x, y)=\sqrt{2} x \sqrt{1-x^{2}-y^{2}} \\
& F_{3}(t, x, y)=\sqrt{2} x y
\end{aligned}
$$


o qual induz a família $g_{t}$.

Valem as seguintes condições sobre $F$ :

1. $O$ rank de $F$ no ponto $\left(\arctan (\sqrt{2}), \frac{\sqrt{2}}{2}, \frac{\sqrt{6}}{6}\right)$ é igual a 2. De fato, se fosse $\operatorname{rank}(F)=3$, então pelo menos um dos menores de $g_{\arctan (\sqrt{2})}$ seria não nulo, o que é impossível, já que $g_{\arctan (\sqrt{2})}$ tem rank 1 no ponto em questão.

2. $D_{2}=J\left(F_{2}, F_{3}\right)=0$ e $D_{3}=J\left(F_{1}, F_{3}\right)=0$ são independentes e definem localmente a curva

$$
y \rightarrow\left(t(y), \frac{\sqrt{2}}{2}, y\right)
$$

onde $t(y)$ é a curva 5.6, que é chamada curva de pinch-points, e é tangente ao plano $t=\arctan (\sqrt{2})$ em $\left(\arctan (\sqrt{2}), \frac{\sqrt{2}}{2}, \frac{\sqrt{6}}{6}\right)$.

3. O contato entre a curva de pinch-points e o plano $t=\arctan (\sqrt{2})$ é precisamente de segunda ordem, pois é fácil ver que este plano é o plano osculador desta curva e, além disso, sua segunda derivada em $y=\frac{\sqrt{6}}{6}$ é não nula.

4. A restrição de $F$ à curva de guarda-chuvas é regular em $\left(\arctan (\sqrt{2}), \frac{\sqrt{2}}{2}, \frac{\sqrt{6}}{6}\right)$. De fato, a matriz jacobiana de $\left(D_{2}, D_{3}, F_{3}\right)$ neste ponto é dada por

$$
\left(\begin{array}{ccc}
0 & -4 \sqrt{3} & 0 \\
-\frac{2 \sqrt{6}}{3} & 0 & 0 \\
0 & \frac{\sqrt{3}}{3} & 1
\end{array}\right)
$$

cujo determinante é $-8 \sqrt{2}$.

Pela proposição 5 de Apéry ([1],pag.71), as 4 condições acima implicam que $F$ é uma confluência de guarda-chuvas. 


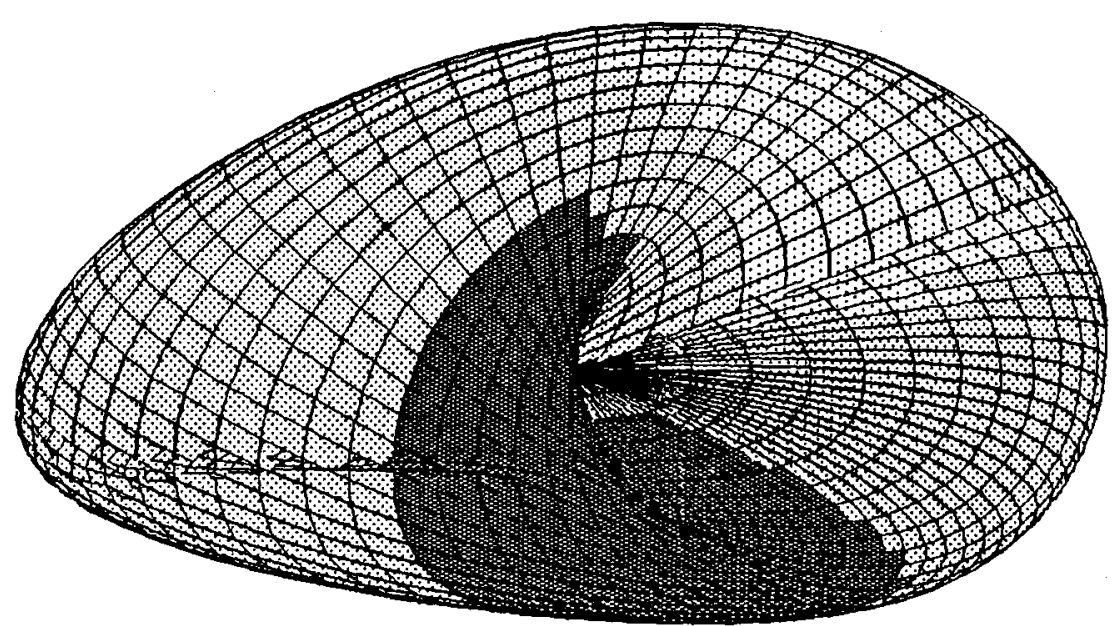

Figura 5.9: Superfície de bifurcação

Observação 5.4 O estudo de diagramas do tipo

$$
M^{2} \stackrel{i}{\rightarrow} \mathbf{E}^{4} \stackrel{\pi_{t}}{\rightarrow} \mathbf{E}^{3},
$$

onde $i$ é imersão justa de $M^{2}$ em $\mathbf{E}^{4}$ e $\pi_{t}$ é uma familia de projeções, é interessante e parece ser um problema em aberto. Outro problema interessante e relacionado com aplicações justas definidas em $M^{2}$ diz respeito ao número de guarda-chuvas destas aplicações. Por exemplo, em [3], Banchoff coloca a seguinte

Conjectura 5.1 Toda aplicação $C^{\infty}$-estável e justa do plano projetivo em $\mathbf{E}^{3}$ ou tem seis ou tem dois guarda-chuvas de Whitney. 


\section{Bibliografia}

[1] Apéry, F., Models of the real projective plane, Braunschweig, Wiesbaden; Vieweg, 1987.

[2] Banchoff, T.F., High codimensional 0-tight maps om spheres, Proc. Amer. Math. Soc. 161, 1971, 193-205.

[3] Banchoff, T.F., Computer animation and the geoemtry of 3- and 4space, Proceedings of the International Congress of Mathematicians, Helsinqui, 1973.

[4] Carmo, M.P. do, Differential geometry of curves and surfaces, Prentice-Hall, Inc., Englewood Cliffs, New Jersey, 1976.

[5] Carmo, M.P. do, Geometria riemanniana, Projeto Euclides, IMPA, Rio de Janeiro, 1988.

[6] Cecil, T.E. \& Ryan, P.J., Tight and taut immersions of manifolds, Research Notes in Mathematics 107, Boston, London, Melbourne, Pitman Publ., 1985.

[7] Cecil, T.E. \& Ryan, P.J., On the number of top-cicles of a tight surface in 3-space, J. London Math. Soc. (2), 30, (1984), 335-341.

[8] Chern, S.S. \& Lashof, R.K., On the total curvature of immersed manifolds, Amer. Journal Math., 79, 1957.

[9] Chern, S.S. \& Lashof, R.K., On the total curvature of immersed manifolds II, Mich. Math. Journal, 5, 1958.

[10] Eilenberg, S. \& Steenrood, N., Foundations of algebraic topology, Princeton University Press, 1952.

[11] Haab, F.A.J., Immersions tendues de surfaces dans $\mathbf{E}^{3}$, Tese de Doutorado, IMPA, 1990. 
[12] Kuiper, N.H., Minimal total absolute curvature for immersions, Inventiones Mathematicae, 10, 209-238, 19700.

[13] Kuiper, N.H., On Convex maps, Nieuw Archief voon Wiskunde (3), $\mathrm{X}, 147-164,1962$.

[14] Kuiper, N.H., On surfaces in euclidean three-space, Bull. Soc. Math. de Belgique, 12, 1960.

[15] Kuiper, N.H., Convex immersions of closed surfaces in $\mathbf{E}^{3}$, Comm. Math. Helv., 35 (1961), 85-92.

[16] Kuiper, N.H., Stable surfaces in euclidean three-space, Math. Scand., 36 (1975), 83-96.

[17] Kuiper, N.H. \& Meeks, W.III, Total curvature for knotted surfaces, Inventiones Mathematicae, 77, 25-69, 1984.

[18] Langevin, R. \& Rosenberg, H., On curvature integrals and knots, Topology, Vol. 15, 1976, 405-416.

[19] Lastufka, W.S., Tight topological immersions of surfaces in Euclidean space, J. Differential Geometry, 16 (1981), 373-400.

[20] Massey, W., Algebraic topology: an introduction, Harcourt, Brace and World, New York, 1967.

[21] Milnor, J., Morse theory, Ann. Math. Study, Princeton University Press, 1963.

[22] Wilmore, T.J., Total curvature in riemannian geometry, Ellis Horwood Limited, West Sussex, England, 1982.

[23] Wilson, J.P., The total absolute curvature of an immersed manifold, J. London Math. Soc., Vol. 40, 362-366, 1965. 